\title{
The global prevalence of depression, suicide ideation, and attempts in the military forces: a systematic review and Meta-analysis of cross sectional studies
}

Yousef Moradi ${ }^{1,2}$, Behnaz Dowran $^{3}$ and Mojtaba Sepandi ${ }^{*}$

\begin{abstract}
Background: Given the wide range of depressive disorders, suicidal ideation and suicide attempts in various military studies around the world, determining the exact prevalence of these disorders in line with health planning as well as care and treatment service designing for military forces can be useful. The aim of the present metaanalysis was to determine the pooled prevalence of depressive disorders, suicide thoughts, and attempts in the military.

Methods: The present systematic review and meta-analysis study was performed based on PRISMA criteria in 5 steps of the search strategy, screening and selection of articles, data extraction, evaluation of article quality and meta-analysis. International databases (PubMed (Medline), Scopus, Web of science, Embase (Elsevier), Psyclnfo (Ovid), Cochrane CENTRAL (Ovid)) were searched using related keywords extracted from Mesh and Emtree. After screening and final selection of articles, data were extracted and qualitative evaluation was performed using the NOS checklist.

Results: The results of meta-analysis showed that the prevalence of depression in active military forces and veterans was 23\% (\%95 Cl: 20-26\%) and 20\% (\%95 Cl: 18-22\%), respectively. In addition, the prevalence of suicidal ideation and attempts in the military was 11\% (\%95 Cl: 10-13\%) and 11\% (\%95 Cl: 9-13\%), respectively. The prevalence of suicide ideation and attempts in drug-using military was 18\% (\%95 Cl: 7-33\%) and 30\% (\%95 Cl: 23$36 \%$ ), respectively. The prevalence of suicidal ideation and attempts in military consuming alcohol were 9\% (\%95 Cl: 4-13\%) and 8\% (\%95 Cl: 7-10\%), respectively. In militaries with AIDS / HIV, the prevalence of suicide attempts was 5\% (\%95 Cl: 4-8\%).
\end{abstract}

Conclusion: Therefore, it is necessary to develop and design training and intervention programs in order to increase the awareness of the military, especially veterans, to prevent the occurrence of suicide and depression.

Keywords: Suicide ideation, Suicide attempts, Depression, Military, Systematic review and Meta-analysis

* Correspondence: msepandi@gmail.com

${ }^{1}$ Health Research Center, Life Style Institute, Baqiyatallah University of

Medical Sciences, Tehran, Iran

Full list of author information is available at the end of the article

(c) The Author(s). 2021 Open Access This article is licensed under a Creative Commons Attribution 4.0 International License, which permits use, sharing, adaptation, distribution and reproduction in any medium or format, as long as you give appropriate credit to the original author(s) and the source, provide a link to the Creative Commons licence, and indicate if changes were made. The images or other third party material in this article are included in the article's Creative Commons licence, unless indicated otherwise in a credit line to the material. If material is not included in the article's Creative Commons licence and your intended use is not permitted by statutory regulation or exceeds the permitted use, you will need to obtain permission directly from the copyright holder. To view a copy of this licence, visit http://creativecommons.org/licenses/by/4.0/. The Creative Commons Public Domain Dedication waiver (http://creativecommons.org/publicdomain/zero/1.0/) applies to the data made available in this article, unless otherwise stated in a credit line to the data. 


\section{Background}

Mental health is one of the basic pillars of health that requires a useful, effective and satisfactory individual life [1]. Promoting the mental health of a society requires the dynamism and growth of that society [2]. Paying attention to mental health in all areas of life, including personal, social and professional ones, is important and debatable. One of the areas in which mental health is concerned is the job and profession. Based on the available findings, mental disorders are one of the most important and significant causes of diseases and it was predicted that in 2020 the share of mental and neurological disorders in the total burden of diseases would increase by 50\% [3-5]. Therefore, attention to mental health is important in all areas of the individual, social and professional life $[6$, 7]. One of the important stressful environmental stimuli that can cause chronic stress and significantly affect people's psyche is the type of the job in which a person is engaged so that if the stress caused by the work environment becomes excessive, it can cause physical and psychological effects on the individual and his/her family. It can be said that it endangers the health of the individual and threatens the organizational goals and leads to a decrease in the quality of the individual's performance. Research has shown that several factors affect job stress [8-10]. These include shift work, or jobs which are full of environmental stress. If a person is not able to cope with the stressors of his/her job, he/she will suffer from multiple physical, psychological and behavioral consequences. In this regard, the military forces of different countries perform different missions according to the conditions of the region and their countries, but during this decade, in order to provide higher defense capability and presence at greater depths and distances away from the origin, military forces need to design and make tools with higher ranges and quality, which need their own engineering and ergonomic requirements [11-13]. One of the most important issues in this field, which can be the first question and has caused intellectual and executive concern of military officials and commanders, is to identify and implement methods to increase the durability and maintain the performance of military personnel so that during increasing mission time, their efficiency will not be disrupted or effectively reduced [14, 15]. This is where the role of military psychology and psychological variables affecting the effectiveness of military forces become clearer [16, 17]. Psychological assessment and mental disorders are very important among military personnel because war, living in operational conditions, multiple combat missions, being away from the family, captivity, wounding and environmental restrictions, as well as cultural differences are always parts of the military life. Therefore, due to this type of lifestyle, burnout, job stress and various mental disorders such as depression and suicide are very common among them $[18,19]$. For this reason, conducting epidemiological and psychological research among military personnel is of great importance. In addition, accurately determining the prevalence of mental disorders in this group can help health policy makers and health professionals to take more effective and appropriate control and treatment measures $[20,21]$. On the other hand, the military forces' awareness of the occurrence of these disorders can be effective in performing appropriate health behaviors, suitable lifestyle changes, and ultimately in preventing further occurrence of these disorders. So far, various descriptive and analytical studies have been conducted in the world with the aim of determining the prevalence of mental disorders, especially depression and suicide in servicemen in various fields such as naval, land and air forces, but the results of these studies were very contradictory. So far, various studies with different sample sizes in the world have been conducted to determine the prevalence of depression and suicide (thoughts or attempted) in the military, but the results of these studies showed the wide prevalence of these consequences in the military and so far, the exact prevalence of them in these communities has not been determined [7, 22-24]. The unavailability of the exact prevalence of depression and suicide in the military prevents the development of appropriate mental health programs and interventions for the military. On the other hand, the burden of these diseases and mental illnesses in the military is still questionable due to the unavailability of an accurate prevalence [25-27]. Accurately determining the prevalence of depression and suicide in the military can help determine the burden of mental illnesses in the military, plan mental health, develop and implement mental health interventions, as well as allocate health resources. Also, it makes health policy makers and the health sector aware of the level of mental illnesses in the military. In this study, the authors aimed to accurately estimate the prevalence of depression, suicide thoughts and attempts in the world's military.

\section{Methods}

This systematic review and meta-analysis was based on the standards Preferred Reporting Items for Systematic Reviews and Meta-Analyzes (PRISMA) and Metaanalyzes of Observational Studies in Epidemiology (MOOSE) [28-30]. The protocol of this study had been registered in the International Prospective Register of 
Systematic Reviews (PROSPERO), under the registration number of CRD42021233973.

\section{Search syntax and search strategy}

This study was a systematic review and meta-analysis that aimed to accurately determine the prevalence of depression, suicide thoughts, and suicide attempts in the military. Finding of articles published from January 1990 to December 2020 was done in 5 electronic databases (PubMed (Medline), Scopus, Web of science, Embase (Elsevier), PsycInfo (Ovid), Cochrane CENTRAL (Ovid)) using the main keywords of Depression (synonymous with "Depressively", "Depressive Disorder", "Depressed", "Depressive Symptoms", "Emotional Depression", "Unipolar Depression", "Neurotic Depression", "Depressive Syndromes", "Endogenous Depression", and "Depressive Neurosis)", suicide thoughts and attempts (with synonyms of "Suicide", "Suicidality", "Attempted Suicide", "Para Suicide", "Completed Suicide", and "Thoughts of Suicide"), as well as Military people (with synonyms of "Armed Forces Personnel", "Military Personnel", "Air Forces Personnel", "Veterans", "Submariners", "Marines", "Navy Personnel", "Sailors", "Soldiers", "Military Deployment", and "Coast Guard ") (Supplement File). Gray Literature-related sites and databases such as Google Scholar, World Health Organization (WHO) were also searched. The search was generally done in google scholar in the advanced section, then the first 10 pages of the results were reviewed and matched with the final selected articles so that any article was not lost. For the World Health Organization website, international or national reports, the references of which were reviewed, were generally searched on the main website using main keywords, i.e. depression and suicide, then the keyword of military was considered in the study. The manual search in this article was performed by checking the reference lists of the articles. In this way, the references of the selected articles were scanned very quickly so that a relevant article would not be missed. In this review articles with English language were included.

\section{Eligibility criteria's}

Inclusion criteria contained the following:

Cross-sectional studies whose main purpose was to estimate and determine the prevalence (frequency or percentage) of depression and suicide (thoughts or attempts) in the military.

Cross-sectional studies that measured depression and suicide (suicidal ideation or attempts) in the military using accredited tools.

Cross-sectional studies in which the study population was military personnel (serving or retired). Individuals who had been employed by the Army, Air Force, and
Navy or retired from any of these organizations. In addition, servicemen who had fought in foreign wars (such as the wars in Afghanistan, Syria, Iraq, and Vietnam) would be considered military forces (active or retired) if surveyed in the selected studies (then they were separately analyzed in subgroup analyzes).

In this review articles with English language were included.

Exclusion criteria contained cross-sectional studies that had reported the desired outcomes (depression and suicide) on a crude average with standard deviation. Their target population was not military and they had not provided a precise definition of the military. In addition, studies other than cross-sectional ones, such as cohort studies, case studies, retrospective, or prospective studies with the cohort base, clinical trials, systematic reviews, letters to the editor, editorial, and survey studies over 5 years were excluded from the research.

\section{Screening and selection of articles}

A definition was not included in the inclusion criteria for measuring suicide (suicide attempts or suicidal ideation) and depression, so the authors decided to screen and select articles, then based on the various tools (like standard questionnaires, the DSM-IV criteria or clinical findings measuring) used in the selected studies to measure depression and suicide, to perform subgroup analysis whose results were presented in the analysis tables.

First, an Endnote library (Version 8) was created to collect articles, remove duplicates, and review titles and abstracts. In the first screening step, the review of titles and abstracts was independently done by one of the researchers (YM) and $10 \%$ of the reviewed articles were randomly reviewed by the second researcher (MS) and the differences were resolved by discussing and referring to the third person (BD) if necessary. The screened references were selected for full-text review if they contained the desired information in their title or abstract. In the next step, the full text was separately reviewed by two of the authors. Data were extracted from the eligible studies and entered into Excel 2016.

\section{Data extraction}

In order to extract the data, first a checklist was prepared with the opinion of experts in relation to the data extracted from the articles and then the data were extracted. Required information included author's name, year of publication of articles, statistical population of study, country of study, type of study, instrument for measuring depression and suicide disorders in the military, sample size, average age of military personnel and quality evaluation score of primary studies. The data 
extraction was independently developed and conducted by two of the authors (YM and MS).

\section{Quality assessment}

Two of the authors (YM and MS) conducted a qualitative evaluation of the studies based on the Newcastle Ottawa Quality Assessment Scale (NOS) checklist [31, 32]. This checklist has designed to evaluate the quality of observational studies, especially cross-sectional ones. This tool examined each study with 6 items in three groups, including: how to select study samples, how to compare and analyze study groups, and how to measure and analyze the desired outcome. Each of these items was given a score of 1 if it was observed in the studies, and the maximum score for each study was 9 points. In case of discrepancies in the score assigned to the published articles and for reaching an agreement, the discussion method and the third researcher (BD) were used.

\section{Statistical methods}

The number of patients with the desired outcome (depression or suicide) was extracted from the total sample size in each of the studies to perform the Metaprop order. In this research, the model of DerSimonian-Liard random effects was used to estimate the pooled prevalence of depression and suicide (estimate of 95\% confidence interval) in military personnel. Cochrane Q and I2 tests were used to investigate the heterogeneity and variance between the studies selected for meta-analysis. According to the Cochrane criteria and I2 index, the amount of heterogeneity was divided into 4 categories: 0 to $40 \%$ (might not be important), 30 to $60 \%$ (may represent moderate heterogeneity), 50 to $90 \%$ (may represent substantial heterogeneity), and finally $75 \%$ and above (considerable heterogeneity) [33-36]. The L'Abbé Plot diagram was used to investigate this heterogeneity. Subgroup analysis was also used to find the source of heterogeneity (gender, service status (active or veteran) and health status of the military as well as sampling types, outcome measurement tools and finally the country). The Funnel Plot diagram and Egger test were used to check and determine the publication bias. The interpretation of the Egger test is that if the $P$ value is significant, it can be interpreted that the publication bias has occurred, otherwise no bias has occurred. In addition, the Funnel diagram was used to express this bias. All analyzes were performed in STATA software, version 16.

\section{Results}

\section{Qualitative results}

After completing the search strategy, and eliminating duplicates in EndNote software, 5275 articles related to depression and 3022 articles related to suicide in the military of the world remained. After screening based on their titles and abstracts, 245 articles on depression and 221 articles on suicide remained in the study. Screening was performed based on the full texts of the articles, and finally 112 articles on depression and 163 articles on suicide were removed. Finally, 133 articles on depression and 58 articles on suicide in the military remained, which entered the meta-analysis. Of the suicide articles, 48 were about suicide attempts and 49 were about suicidal ideation. Some of these articles reported both suicidal ideation and suicide attempts (Fig. 1). All characteristics extracted from selected studies were separately reported in Tables 1 and 2 based on the outcome of depression and suicide.

\section{Quantitative analysis}

\section{Prevalence of depression in the all military}

Initially, the studies were divided into two groups: the active duty military community and the veteran's community in terms of the study population. Then, separate analyzes were performed for each of these communities and the prevalence of depression in each was metaanalyzed. Of the 133 final selected cross-sectional studies, 80 were in the veterans and 100 were in the active duty military personnel.

\section{Prevalence of depression in the active duty military}

In these studies, $1,278,837$ employees of the active or serving military had been examined, of whom 273,173 had depression. After combining the results of these studies, the overall pooled prevalence of depression in the active or in-service military was $23 \%$ with a confidence interval of 20 to $26 \%$. The percentage of heterogeneity was $99.91 \%$ which was statistically significant (Table 3).

The pooled prevalence of depression was 21\% (\% 95 CI; 18-25\%) in studies where the sampling method was the available one (convinces sampling). A total of 67 studies used this type of sampling method, which had examined a total of 939,796 active members, of whom 21,7487 had been considered depressed. In addition, 33 studies with a sample size of 339,041 people had used the random sampling method to collect their samples. After combining these studies, the pooled prevalence of depression was estimated to be $26 \%$ (\% $95 \mathrm{CI} ; 19-32 \%$ ) (Table 3).

In this meta-analysis, the pooled prevalence of depression in active duty military personnel was also calculated based on the location and the results were reported in Table 3. The results showed that the pooled prevalence of depression in active air, land, and naval forces was 20\% (\% 95 CI; 9-33\%), 22\% (\% 95 CI; 20-23\%), and 31\% (\% $95 \mathrm{CI} ; 16-48 \%$ ), respectively. In 53 cross-sectional studies, it had not been specified that in which military unit, the study population was serving and it had been 

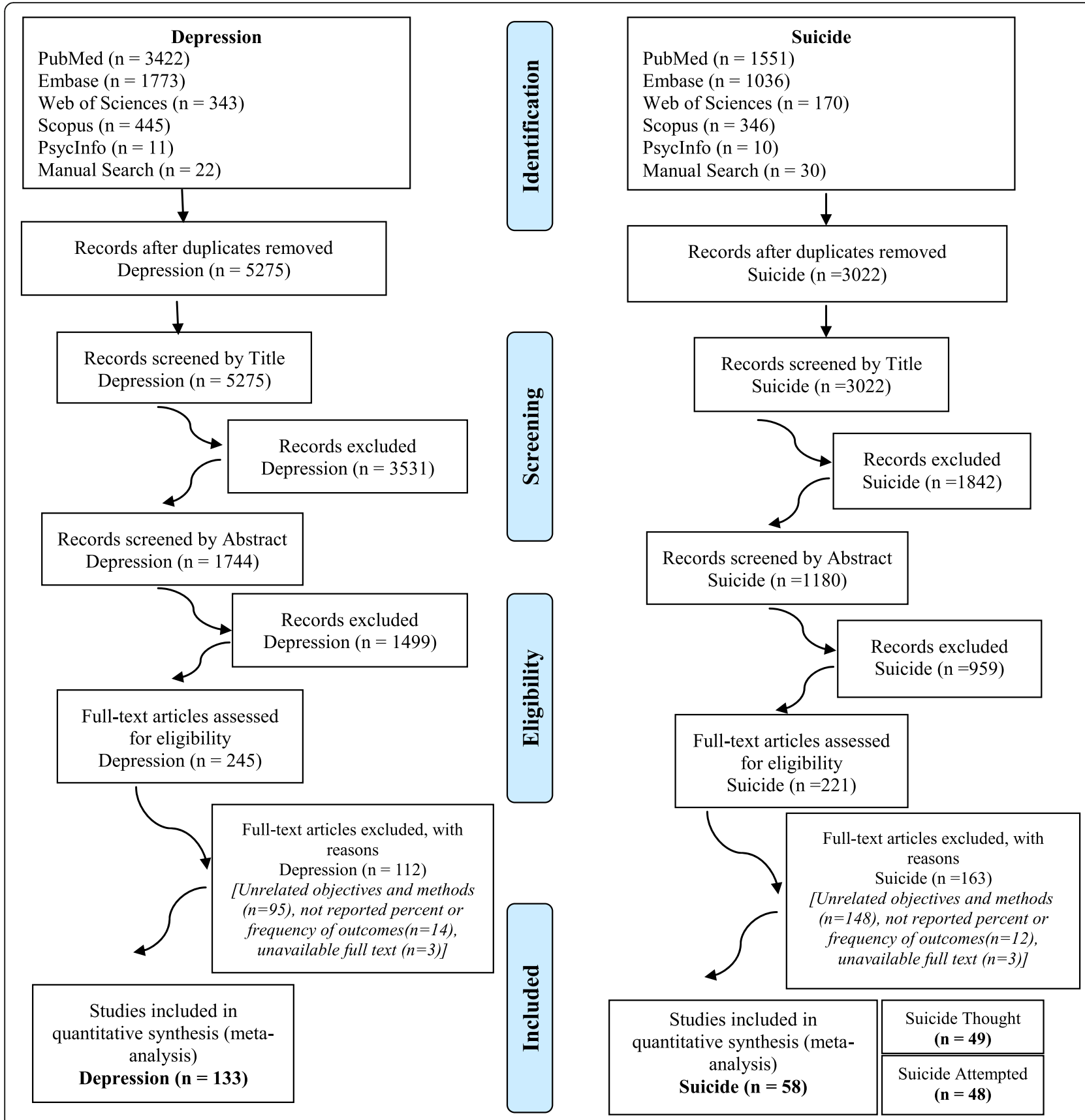

Fig. 1 The Search Strategy Outputs and Screening Process based on Title, Abstract, and Full Text

mentioned as military forces in that studies, so, a group called military forces was formed, the sample size of which was equal to 201,624 active military personnel of whom 65,158 people were depressed. The pooled prevalence of depression after a combination of these studies was $22 \%$ (\% $95 \mathrm{CI}$; 16-28\%) (Table 3).

The pooled prevalence of depression in active militaries with HIV was 15\% (\% 95 CI; 3-36\%), in active militaries with substance use was 37\% (\% 95 CI; 36-39\%), in militaries using alcohol was equal to $29 \%$ (\% 95 CI; $13-$
$47 \%)$ and finally in healthy and disease-free military members was equal to $22 \%$ (\% 95 CI; 20-25\%) (Table 3).

The pooled prevalence of depression in the active military varied by gender. A total of 71 cross-sectional studies had not identified the gender of the study population while 20 and 9 studies had been performed on military men and women, respectively. In studies that had not specified gender, the sample size was $1,163,273$ people, of whom 221,910 individuals were depressed. The sample size in cross-sectional studies on military men and 
Table 1 The study characteristics of included studies about depression

\begin{tabular}{|c|c|c|c|c|c|c|c|c|}
\hline $\begin{array}{l}\text { Authors } \\
\text { (Years) }\end{array}$ & Country & $\begin{array}{l}\text { Type of } \\
\text { Sampling } \\
\text { (Type of } \\
\text { Study) }\end{array}$ & Study Population & Depression Assessment Method & $\begin{array}{l}\text { Age } \\
\text { (Mean) }\end{array}$ & $\begin{array}{l}\text { Sample } \\
\text { size }\end{array}$ & $\begin{array}{l}\text { Prevalence } \\
\text { of } \\
\text { Depression } \\
\text { (\%) } \\
\end{array}$ & $\begin{array}{l}\text { NOS } \\
\text { Score }\end{array}$ \\
\hline $\begin{array}{l}\text { Tredgold, R. } \\
\text { F. (1941)(65) }\end{array}$ & UK & $\begin{array}{l}\text { Convenience } \\
\text { Sampling } \\
\text { (CS) }\end{array}$ & Army men & Clinical Symptoms (Interviews) & - & 274 & $70(25.54 \%)$ & 6 \\
\hline $\begin{array}{l}\text { Helzer, J. E. } \\
\text { et al. (1976) } \\
\text { (66) }\end{array}$ & USA & $\begin{array}{l}\text { Random } \\
\text { Sampling } \\
\text { (CS) }\end{array}$ & Army men & Clinical Symptoms (Interviews) & - & 470 & $122(26 \%)$ & 7 \\
\hline $\begin{array}{l}\text { Levine, M. E. } \\
\text { (1982) (67) }\end{array}$ & USA & $\begin{array}{l}\text { Convenience } \\
\text { Sampling } \\
\text { (CS) }\end{array}$ & Army men & Beck Depression Inventory (BDI) & 17 & 200 & $36(18 \%)$ & 6 \\
\hline $\begin{array}{l}\text { Deeken, M. G. } \\
\text { et al. (1987) } \\
\text { (68) }\end{array}$ & USA & $\begin{array}{l}\text { Convenience } \\
\text { Sampling } \\
\text { (CS) }\end{array}$ & Army men & Zung Self-Rating Depression Scale & - & 298 & 47 (15.77\%) & 7 \\
\hline $\begin{array}{l}\text { Ritchie, E. C. } \\
\text { et al. (1992) } \\
\text { (69) }\end{array}$ & USA & $\begin{array}{l}\text { Random } \\
\text { Sampling } \\
\text { (CS) }\end{array}$ & Army men with HIV & $\begin{array}{l}\text { Clinical Symptoms (Interviews) DSM- } \\
\text { III-R }\end{array}$ & - & 50 & $21(42 \%)$ & 7 \\
\hline $\begin{array}{l}\text { Brown, G. R. } \\
\text { et al. (1993) } \\
\text { (70) }\end{array}$ & USA & $\begin{array}{l}\text { Random } \\
\text { Sampling } \\
\text { (CS) }\end{array}$ & $\begin{array}{l}\text { Air Forces men with } \\
\text { HIV }\end{array}$ & $\begin{array}{l}\text { Structured Interview Guide for the } \\
\text { Hamilton Anxiety and Depression } \\
\text { Scales (SIGH-AD) }\end{array}$ & 35 & 442 & $99(22.4 \%)$ & 8 \\
\hline \multirow{3}{*}{$\begin{array}{l}\text { McCarroll, J. } \\
\text { E. et al. } \\
(1993)(71)\end{array}$} & \multirow[t]{3}{*}{ USA } & \multirow{3}{*}{$\begin{array}{l}\text { Convenience } \\
\text { Sampling } \\
\text { (CS) }\end{array}$} & \multirow{3}{*}{$\begin{array}{l}\text { Army men and } \\
\text { women }\end{array}$} & \multirow[t]{3}{*}{ Clinical Symptoms (Interviews) } & \multirow[t]{3}{*}{25.4} & 1835 & $87(4.7 \%)$ & \multirow[t]{3}{*}{8} \\
\hline & & & & & & Male(1565) & $59(3.8 \%)$ & \\
\hline & & & & & & Female(270) & $52(19.3 \%)$ & \\
\hline $\begin{array}{l}\text { Perconte, S. } \\
\text { T. et al. } \\
\text { (1993) (72) }\end{array}$ & Russia & $\begin{array}{l}\text { Convenience } \\
\text { Sampling } \\
\text { (CS) }\end{array}$ & $\begin{array}{l}\text { Army men and } \\
\text { women }\end{array}$ & Beck Depression Inventory (BDI) & 29.25 & 591 & $146(24.70 \%)$ & 7 \\
\hline $\begin{array}{l}\text { Serfaty, E. } \\
\text { et al. (1995) } \\
\text { (73) }\end{array}$ & Argentina & $\begin{array}{l}\text { Random } \\
\text { Sampling } \\
\text { (CS) }\end{array}$ & $\begin{array}{l}\text { Army men and } \\
\text { women }\end{array}$ & NR & NR & 553 & $25(4.5 \%)$ & 7 \\
\hline $\begin{array}{l}\text { Lish, J. D. } \\
\text { et al. (1996) } \\
\text { (74) }\end{array}$ & USA & $\begin{array}{l}\text { Random } \\
\text { Sampling } \\
\text { (CS) }\end{array}$ & $\begin{array}{l}\text { Army men and } \\
\text { women }\end{array}$ & $\begin{array}{l}\text { Brief self-report questionnaire } \\
\text { (SCRENNER) }\end{array}$ & 21.2 & 669 & $38(5.81 \%)$ & 7 \\
\hline $\begin{array}{l}\text { Long, N. et al. } \\
\text { (1996) (75) }\end{array}$ & $\begin{array}{l}\text { New } \\
\text { Zealand }\end{array}$ & $\begin{array}{l}\text { Random } \\
\text { Sampling } \\
\text { (CS) }\end{array}$ & Army men & Beck Depression Inventory (BDI) & 50 & 751 & $11(1.46 \%)$ & 7 \\
\hline $\begin{array}{l}\text { Schwartz, D. } \\
\text { A. et al. } \\
\text { (1997) (76) }\end{array}$ & USA & $\begin{array}{l}\text { Random } \\
\text { Sampling } \\
\text { (CS) }\end{array}$ & $\begin{array}{l}\text { Non-Persian Gulf } \\
\text { War (PGW) military } \\
\text { personnel }\end{array}$ & Self-report & - & 923 & $157(17 \%)$ & 6 \\
\hline $\begin{array}{l}\text { Schwartz, D. } \\
\text { A. et al. } \\
\text { (1997) (76) }\end{array}$ & USA & $\begin{array}{l}\text { Random } \\
\text { Sampling } \\
\text { (CS) }\end{array}$ & $\begin{array}{l}\text { Persian Gulf War } \\
\text { (PGW) military } \\
\text { personnel }\end{array}$ & Self-report & - & 923 & $99(10.9 \%)$ & 6 \\
\hline $\begin{array}{l}\text { David, D. } \\
\text { et al. (1999) } \\
\text { (77) }\end{array}$ & Croatia & $\begin{array}{l}\text { Convenience } \\
\text { Sampling } \\
\text { (CS) }\end{array}$ & $\begin{array}{l}\text { Veterans after } \\
\text { participation in } \\
\text { Homeland War in } \\
\text { Croatia }\end{array}$ & $\begin{array}{l}\text { The Structured Clinical Interview } \\
\text { Diagnostic and Statistical Manual } \\
\text { (SCID) }\end{array}$ & 36.2 & 91 & $35(38.5 \%)$ & 7 \\
\hline $\begin{array}{l}\text { Hankin, C. S. } \\
\text { et al. (1999) } \\
\text { (78) }\end{array}$ & USA & $\begin{array}{l}\text { Random } \\
\text { Sampling } \\
\text { (CS) }\end{array}$ & Men Veterans & $\begin{array}{l}\text { Center for Epidemiologic Studies } \\
\text { Depression Scale (CES-D Scale) }\end{array}$ & 62 & 2160 & $676(31.3 \%)$ & 7 \\
\hline \multirow{3}{*}{$\begin{array}{l}\text { Hourani, L. L. } \\
\text { et al. (1999) } \\
\text { (79) }\end{array}$} & \multirow[t]{3}{*}{ USA } & \multirow{3}{*}{$\begin{array}{l}\text { Random } \\
\text { Sampling } \\
\text { (CS) }\end{array}$} & \multirow{3}{*}{$\begin{array}{l}\text { Men and Women in } \\
\text { the Navy and Marine } \\
\text { Corps }\end{array}$} & \multirow{3}{*}{$\begin{array}{l}\text { Center for Epidemiologic Studies } \\
\text { Depression Scale (CES-D Scale) }\end{array}$} & \multirow[t]{3}{*}{$20-64$} & 782 & $125(16.08 \%)$ & \multirow[t]{3}{*}{7} \\
\hline & & & & & & Male (321) & $29(9 \%)$ & \\
\hline & & & & & & $\begin{array}{l}\text { Female } \\
(452)\end{array}$ & $99(22 \%)$ & \\
\hline $\begin{array}{l}\text { Curran, G. M. } \\
\text { et al. (2000) } \\
\text { (80) }\end{array}$ & USA & $\begin{array}{l}\text { Random } \\
\text { Sampling } \\
\text { (CS) }\end{array}$ & Men Veterans & (Beck Depression Inventory)BDI( & 43 & 298 & $116(39 \%)$ & 7 \\
\hline Menon, A. S. & USA & Convenience & Men Veterans & The Structured Clinical Interview for & 55 & 295 & $59(22.8 \%)$ & 6 \\
\hline
\end{tabular}


Table 1 The study characteristics of included studies about depression (Continued)

\begin{tabular}{|c|c|c|c|c|c|c|c|c|}
\hline $\begin{array}{l}\text { Authors } \\
\text { (Years) }\end{array}$ & Country & $\begin{array}{l}\text { Type of } \\
\text { Sampling } \\
\text { (Type of } \\
\text { Study) }\end{array}$ & Study Population & Depression Assessment Method & $\begin{array}{l}\text { Age } \\
\text { (Mean) }\end{array}$ & $\begin{array}{l}\text { Sample } \\
\text { size }\end{array}$ & $\begin{array}{l}\text { Prevalence } \\
\text { of } \\
\text { Depression } \\
(\%)\end{array}$ & $\begin{array}{l}\text { NOS } \\
\text { Score }\end{array}$ \\
\hline $\begin{array}{l}\text { et al. (2000) } \\
\text { (81) }\end{array}$ & & $\begin{array}{l}\text { Sampling } \\
\text { (CS) }\end{array}$ & & DSM-III-R (SCID-III-R) & & & & \\
\hline $\begin{array}{l}\text { Kozaric- } \\
\text { Kovacic, D. } \\
\text { et al. (2001) } \\
\text { (82) }\end{array}$ & Croatia & $\begin{array}{l}\text { Random } \\
\text { Sampling } \\
\text { (CS) }\end{array}$ & Men Veterans & $\begin{array}{l}\text { The Hamilton Depression Rating Scale } \\
\text { (HAMD) }\end{array}$ & 34 & 249 & 77 (31\%) & 7 \\
\hline $\begin{array}{l}\text { Sayar, K. et al. } \\
(2001)(83)\end{array}$ & Turkey & $\begin{array}{l}\text { Random } \\
\text { Sampling } \\
\text { (CS) }\end{array}$ & Men Soldiers & (Beck Depression Inventory)BDI( & 22.7 & 40 & $13(32.5 \%)$ & 7 \\
\hline $\begin{array}{l}\text { Hunter, C. } \\
\text { L.et al. } \\
\text { (2002)(84) }\end{array}$ & USA & $\begin{array}{l}\text { Random } \\
\text { Sampling } \\
\text { (CS) }\end{array}$ & Active Duty & $\begin{array}{l}\text { The Patient Health Questionnaire } \\
\text { (PHQ) (the self-report version of the } \\
\text { PRIME-MD) }\end{array}$ & 54.15 & 337 & $19(5.6 \%)$ & 7 \\
\hline \multirow{2}{*}{$\begin{array}{l}\text { Karel, M. J. } \\
\text { et al. } \\
(2002)(85)\end{array}$} & \multirow[t]{2}{*}{ USA } & \multirow{2}{*}{$\begin{array}{l}\text { Random } \\
\text { Sampling } \\
\text { (Survey } \\
\text { Study) }\end{array}$} & \multirow[t]{2}{*}{ Men Veterans } & $\begin{array}{l}\text { The Geriatric Depression Scale (GDS)- } \\
15 \text { item }\end{array}$ & 69.7 & 967 & $236(24.4 \%)$ & \multirow[t]{2}{*}{7} \\
\hline & & & & $\begin{array}{l}\text { Hamilton Depression Rating Scale } \\
\text { (HDRS)-24 item }\end{array}$ & 69.7 & 967 & $94(9.7 \%)$ & \\
\hline $\begin{array}{l}\text { Kilbourne, A. } \\
\text { M. et al. } \\
\text { (2002)(86) }\end{array}$ & USA & $\begin{array}{l}\text { Random } \\
\text { Sampling } \\
\text { (CS) }\end{array}$ & $\begin{array}{l}\text { Veterans with HIV } \\
\text { infection }\end{array}$ & $\begin{array}{l}\text { The } 10 \text {-item Centers for Epidemiologic } \\
\text { Studies Depression Scale (CES-D) }\end{array}$ & 49 & 881 & $405(46 \%)$ & 7 \\
\hline $\begin{array}{l}\text { Lehman, C. L. } \\
\text { et al. } \\
\text { (2002)(87) }\end{array}$ & USA & $\begin{array}{l}\text { Convenience } \\
\text { Sampling } \\
\text { (CS) }\end{array}$ & $\begin{array}{l}\text { Veterans with } \\
\text { Hepatitis C }\end{array}$ & The Beck Depression Inventory (BDI) & 49 & 120 & $53(44.2 \%)$ & 6 \\
\hline $\begin{array}{l}\text { Muir, A. J. } \\
\text { et al. } \\
(2002)(88)\end{array}$ & USA & $\begin{array}{l}\text { Convenience } \\
\text { Sampling } \\
\text { (CS) }\end{array}$ & $\begin{array}{l}\text { Veterans with } \\
\text { Hepatitis C }\end{array}$ & $\begin{array}{l}\text { The Center for Epidemiological } \\
\text { Studies Depression (CES-D) scale }\end{array}$ & 47.3 & 100 & $12(12 \%)$ & 6 \\
\hline $\begin{array}{l}\text { Nguyen, H. A. } \\
\text { et al. } \\
(2002)(89)\end{array}$ & USA & $\begin{array}{l}\text { Convenience } \\
\text { Sampling } \\
\text { (CS) }\end{array}$ & $\begin{array}{l}\text { Veterans with } \\
\text { Hepatitis C }\end{array}$ & Clinical Symptoms (Interviews) & 46.5 & 118 & $73(62 \%)$ & 6 \\
\hline $\begin{array}{l}\text { Black, D. W. } \\
\text { et al. } \\
(2004)(90)\end{array}$ & USA & $\begin{array}{l}\text { Convenience } \\
\text { Sampling } \\
\text { (CS) }\end{array}$ & Veterans & $\begin{array}{l}\text { Clinical Symptoms (Interviews) DSM- } \\
\text { III-R }\end{array}$ & 39.3 & 602 & $192(32 \%)$ & 6 \\
\hline $\begin{array}{l}\text { Gerson, S. } \\
\text { et al. (2004) } \\
\text { (91) }\end{array}$ & USA & $\begin{array}{l}\text { Convenience } \\
\text { Sampling } \\
\text { (CS) }\end{array}$ & $\begin{array}{l}\text { Elderly veterans } \\
\text { (Male) }\end{array}$ & Mental Health Inventory (MHI) & 69.6 & 839 & $273(32.5 \%)$ & 8 \\
\hline $\begin{array}{l}\text { Rowan, P. J. } \\
\text { et al. } \\
(2004)(92)\end{array}$ & USA & $\begin{array}{l}\text { Convenience } \\
\text { Sampling } \\
\text { (CS) }\end{array}$ & $\begin{array}{l}\text { Veterans with } \\
\text { Hepatitis C }\end{array}$ & $\begin{array}{l}\text { The Zung Self-report Depression Scale } \\
\text { (SDS) }\end{array}$ & 51 & 580 & $93(16 \%)$ & 7 \\
\hline $\begin{array}{l}\text { Smith, T. C. } \\
\text { et al. } \\
(2004)(93)\end{array}$ & USA & $\begin{array}{l}\text { Random } \\
\text { Sampling } \\
\text { (CS) }\end{array}$ & US Military & $\begin{array}{l}\text { The PRIME-MD } \\
\text { Patient Health Questionnaire (PHQ) }\end{array}$ & 55 & 8893 & $1642(18.5 \%)$ & 8 \\
\hline $\begin{array}{l}\text { Vafaee, } \\
\text { B.et al. } \\
(2004)(94)\end{array}$ & Iran & $\begin{array}{l}\text { Convenience } \\
\text { Sampling } \\
\text { (CS) }\end{array}$ & $\begin{array}{l}\text { Disabled veterans } \\
\text { male }\end{array}$ & $\begin{array}{l}\text { The Zung Self-report Depression Scale } \\
\text { (SDS) }\end{array}$ & 38 & 100 & $71(71 \%)$ & 5 \\
\hline $\begin{array}{l}\text { Forman- } \\
\text { Hoffman, V. L. } \\
\text { et al. (2005) } \\
\text { (95) }\end{array}$ & USA & $\begin{array}{l}\text { Convenience } \\
\text { Sampling } \\
\text { (CS) }\end{array}$ & Veterans & $\begin{array}{l}\text { Structured Clinical Interview for DSM } \\
\text { Disorders (SCID-IV) }\end{array}$ & 39.1 & 602 & $85(14.11 \%)$ & 6 \\
\hline \multirow{2}{*}{$\begin{array}{l}\text { Goulet, J. L. } \\
\text { et al. } \\
(2005)(96)\end{array}$} & \multirow[t]{2}{*}{ USA } & \multirow{2}{*}{$\begin{array}{l}\text { Convenience } \\
\text { Sampling } \\
\text { (Re) }\end{array}$} & Veterans with HIV & - & 47.1 & 20,627 & $5776(28 \%)$ & \multirow[t]{2}{*}{7} \\
\hline & & & $\begin{array}{l}\text { Veterans with } \\
\text { Hepatitis C }\end{array}$ & - & 46.9 & 4489 & 1975 (44\%) & \\
\hline $\begin{array}{l}\text { Rowan, P. J. } \\
\text { et al. } \\
\text { (2005)(97) }\end{array}$ & USA & $\begin{array}{l}\text { Convenience } \\
\text { Sampling } \\
\text { (CS) }\end{array}$ & $\begin{array}{l}\text { Veterans with } \\
\text { Hepatitis C }\end{array}$ & The Beck Depression Inventory (BDI) & 52 & 62 & $6(10 \%)$ & 5 \\
\hline $\begin{array}{l}\text { Williams, R. } \\
\text { M. et al. }\end{array}$ & USA & $\begin{array}{l}\text { Convenience } \\
\text { Sampling }\end{array}$ & $\begin{array}{l}\text { Veterans with } \\
\text { Multiple sclerosis }\end{array}$ & The Beck Depression Inventory (BDI) & 55.1 & 451 & $100(22.2 \%)$ & 7 \\
\hline
\end{tabular}


Table 1 The study characteristics of included studies about depression (Continued)

\begin{tabular}{|c|c|c|c|c|c|c|c|c|}
\hline $\begin{array}{l}\text { Authors } \\
\text { (Years) }\end{array}$ & Country & $\begin{array}{l}\text { Type of } \\
\text { Sampling } \\
\text { (Type of } \\
\text { Study) }\end{array}$ & Study Population & Depression Assessment Method & $\begin{array}{l}\text { Age } \\
\text { (Mean) }\end{array}$ & $\begin{array}{l}\text { Sample } \\
\text { size }\end{array}$ & $\begin{array}{l}\text { Prevalence } \\
\text { of } \\
\text { Depression } \\
(\%)\end{array}$ & $\begin{array}{l}\text { NOS } \\
\text { Score }\end{array}$ \\
\hline$\overline{(2005)(98)}$ & & (CS) & & & & & & \\
\hline $\begin{array}{l}\text { Xiong, } \mathrm{H} \text {. } \\
\text { et al. } \\
(2005)(99)\end{array}$ & China & $\begin{array}{l}\text { Random } \\
\text { Sampling } \\
\text { (CS) }\end{array}$ & $\begin{array}{l}\text { Young adult males } \\
\text { during their } 8 \text { week } \\
\text { field military training }\end{array}$ & $\begin{array}{l}\text { The Zung Self-report Depression Scale } \\
\text { (SDS) }\end{array}$ & 20 & 1107 & $279(25.2 \%)$ & 6 \\
\hline $\begin{array}{l}\text { Grieger, T. A. } \\
\text { et al. } \\
(2006)(100)\end{array}$ & USA & $\begin{array}{l}\text { Convenience } \\
\text { Sampling } \\
\text { (CS) }\end{array}$ & $\begin{array}{l}\text { U.S. soldiers were } \\
\text { injured in combat }\end{array}$ & $\begin{array}{l}\text { The nine-item Patient Health Ques- } \\
\text { tionnaire depression scale }\end{array}$ & 26.94 & 301 & $28(9.3 \%)$ & 5 \\
\hline $\begin{array}{l}\text { Hoge, C. W. } \\
\text { et al. } \\
(2006)(101)\end{array}$ & USA & $\begin{array}{l}\text { Random } \\
\text { Sampling } \\
\text { (CS) }\end{array}$ & $\begin{array}{l}\text { Army soldiers and } \\
\text { Marines }\end{array}$ & $\begin{array}{l}\text { The PRIME-MD } \\
\text { Patient Health Questionnaire (PHQ) }\end{array}$ & 31.2 & 303,905 & $\begin{array}{l}15,930 \\
(5.24 \%)\end{array}$ & 8 \\
\hline $\begin{array}{l}\text { Kress, A. M. } \\
\text { et al. } \\
(2006)(102)\end{array}$ & USA & $\begin{array}{l}\text { Random } \\
\text { Sampling } \\
\text { (CS) }\end{array}$ & $\begin{array}{l}\text { U.S. Military } \\
\text { personnel }\end{array}$ & Burnam Screen & - & 4227 & $844(20 \%)$ & 8 \\
\hline $\begin{array}{l}\text { Pflanz, S. E. } \\
\text { et al. } \\
(2006)(103)\end{array}$ & USA & $\begin{array}{l}\text { Convenience } \\
\text { Sampling } \\
\text { (CS) }\end{array}$ & Military Personnel & Depression Checklist & 28.7 & 780 & $141(18 \%)$ & 7 \\
\hline $\begin{array}{l}\text { Dove, M. B. } \\
\text { et al. } \\
(2007)(104)\end{array}$ & USA & $\begin{array}{l}\text { Convenience } \\
\text { Sampling } \\
\text { (CS) }\end{array}$ & $\begin{array}{l}\text { Women Entering a } \\
\text { Military Substance } \\
\text { Use Disorder }\end{array}$ & Depression Checklist & - & 86 & $67(78 \%)$ & 5 \\
\hline $\begin{array}{l}\text { Kolkow, T. T. } \\
\text { et al. } \\
(2007)(105)\end{array}$ & USA & $\begin{array}{l}\text { Convenience } \\
\text { Sampling } \\
\text { (CS) }\end{array}$ & Army soldiers & $\begin{array}{l}\text { The PRIME-MD } \\
\text { Patient Health Questionnaire (PHQ) }\end{array}$ & 34.30 & 100 & $5(5 \%)$ & 5 \\
\hline \multirow{3}{*}{$\begin{array}{l}\text { Warner, C. M. } \\
\text { et al. } \\
(2007)(106)\end{array}$} & \multirow[t]{3}{*}{ USA } & \multirow{3}{*}{$\begin{array}{l}\text { Convenience } \\
\text { Sampling } \\
\text { (CS) }\end{array}$} & Military Personnel & \multirow{3}{*}{$\begin{array}{l}\text { The PRIME-MD } \\
\text { Patient Health Questionnaire (PHQ) }\end{array}$} & 20.9 & 1090 & $173(15.9 \%)$ & 6 \\
\hline & & & Male & & 20.9 & 955 & $143(15 \%)$ & \\
\hline & & & Female & & 21 & 135 & $30(22.2 \%)$ & \\
\hline \multirow{2}{*}{$\begin{array}{l}\text { Hoge, C. W. } \\
\text { et al. (2008) } \\
(107)\end{array}$} & \multirow[t]{2}{*}{ USA } & \multirow{2}{*}{$\begin{array}{l}\text { Convenience } \\
\text { Sampling } \\
\text { (CS) }\end{array}$} & Army individual & \multirow{2}{*}{$\begin{array}{l}\text { The PRIME-MD } \\
\text { Patient Health Questionnaire (PHQ) }\end{array}$} & \multirow[t]{2}{*}{ - } & 1885 & $275(15 \%)$ & \multirow[t]{2}{*}{6} \\
\hline & & & Marine individual & & & 775 & $114(14.7 \%)$ & \\
\hline $\begin{array}{l}\text { Iversen, A. C. } \\
\text { et al. } \\
(2009)(108)\end{array}$ & UK & $\begin{array}{l}\text { Random } \\
\text { Sampling } \\
\text { (CS) }\end{array}$ & $\begin{array}{l}\text { UK military } \\
\text { personnel in service } \\
\text { at the time of the } \\
2003 \text { Iraq War }\end{array}$ & $\begin{array}{l}\text { The PRIME-MD } \\
\text { Patient Health Questionnaire (PHQ) }\end{array}$ & 35 & 821 & $223(27.2 \%)$ & 8 \\
\hline \multirow[t]{3}{*}{$\begin{array}{l}\text { Kline, A. et al. } \\
(2009)(109)\end{array}$} & \multirow[t]{3}{*}{ USA } & \multirow[t]{3}{*}{$\begin{array}{l}\text { Convenience } \\
\text { Sampling } \\
\text { (CS) }\end{array}$} & $\begin{array}{l}\text { Vietnam veterans } \\
\text { with Substance Use } \\
\text { Disorder }\end{array}$ & \multirow[t]{3}{*}{ SCID DSM-IV Diagnoses } & 55.20 & 82 & $39(47.9 \%)$ & \multirow[t]{3}{*}{8} \\
\hline & & & $\begin{array}{l}\text { Post-Vietnam } \\
\text { veterans with } \\
\text { Substance Use } \\
\text { Disorder }\end{array}$ & & 46.76 & 236 & $131(55.4 \%)$ & \\
\hline & & & $\begin{array}{l}\text { Persian Gulf veterans } \\
\text { with Substance Use } \\
\text { Disorder }\end{array}$ & & 34 & 55 & $33(59.5 \%)$ & \\
\hline $\begin{array}{l}\text { Rehn, L. M. } \\
\text { et al. } \\
(2009)(110)\end{array}$ & Finland & $\begin{array}{l}\text { Convenience } \\
\text { Sampling } \\
\text { (CS) }\end{array}$ & $\begin{array}{l}\text { Male Finnish military } \\
\text { conscripts }\end{array}$ & The Beck Depression Inventory (BDI) & 20 & 126 & $4(3.2 \%)$ & 6 \\
\hline $\begin{array}{l}\text { Rukskul, l. } \\
\text { et al. (2009) } \\
\text { (111) }\end{array}$ & Thailand & $\begin{array}{l}\text { Convenience } \\
\text { Sampling } \\
\text { (CS) }\end{array}$ & Thai army personnel & Clinical Symptoms (Interviews) & 45 & 1729 & $186(10.75 \%)$ & 5 \\
\hline $\begin{array}{l}\text { Rukskul, I. } \\
(2010)(112)\end{array}$ & Thailand & $\begin{array}{l}\text { Convenience } \\
\text { Sampling } \\
\text { (CS) }\end{array}$ & Thai army personnel & Clinical Symptoms (Interviews) & 45 & 213 & $7(3.3 \%)$ & 5 \\
\hline $\begin{array}{l}\text { Fikretoglu, D. } \\
\text { et al. } \\
(2010)(113)\end{array}$ & Canada & $\begin{array}{l}\text { Convenience } \\
\text { Sampling } \\
\text { (CS) }\end{array}$ & $\begin{array}{l}\text { Canadian } \\
\text { Community Health } \\
\text { Survey-Canadian } \\
\text { Forces }\end{array}$ & $\begin{array}{l}\text { Diagnostic and Statistical Manual of } \\
\text { Mental Disorders-IV (DSM-IV) }\end{array}$ & - & 8441 & $1257(14.9 \%)$ & 8 \\
\hline
\end{tabular}


Table 1 The study characteristics of included studies about depression (Continued)

\begin{tabular}{|c|c|c|c|c|c|c|c|c|}
\hline $\begin{array}{l}\text { Authors } \\
\text { (Years) }\end{array}$ & Country & $\begin{array}{l}\text { Type of } \\
\text { Sampling } \\
\text { (Type of } \\
\text { Study) }\end{array}$ & Study Population & Depression Assessment Method & $\begin{array}{l}\text { Age } \\
\text { (Mean) }\end{array}$ & $\begin{array}{l}\text { Sample } \\
\text { size }\end{array}$ & $\begin{array}{l}\text { Prevalence } \\
\text { of } \\
\text { Depression } \\
(\%)\end{array}$ & $\begin{array}{l}\text { NOS } \\
\text { Score }\end{array}$ \\
\hline & & & KlineKline(CCHS-CF) & & & & & \\
\hline \multirow{3}{*}{$\begin{array}{l}\text { Haskell, S. G. } \\
\text { et al. } \\
(2010)(114)\end{array}$} & \multirow[t]{3}{*}{ USA } & \multirow{3}{*}{$\begin{array}{l}\text { Convenience } \\
\text { Sampling } \\
\text { (CS) }\end{array}$} & \multirow{3}{*}{$\begin{array}{l}\text { War Veterans of Iraq } \\
\text { and Afghanistan }\end{array}$} & \multirow[t]{3}{*}{ Clinical Symptoms (Interviews) } & 32 & Total (1229) & $472(38.4 \%)$ & \multirow[t]{3}{*}{7} \\
\hline & & & & & 32 & Male (1032) & 380 (36.8\%) & \\
\hline & & & & & 30 & $\begin{array}{l}\text { Female } \\
(197)\end{array}$ & $92(46.7 \%)$ & \\
\hline \multirow{3}{*}{$\begin{array}{l}\text { Luxton, D. D. } \\
\text { et al. } \\
(2010)(115)\end{array}$} & \multirow[t]{3}{*}{ USA } & \multirow{3}{*}{$\begin{array}{l}\text { Convenience } \\
\text { Sampling } \\
\text { (CS) }\end{array}$} & \multirow{3}{*}{$\begin{array}{l}\text { Active duty Soldiers } \\
\text { between March } \\
2006 \text { and July } 2009 .\end{array}$} & \multirow{3}{*}{$\begin{array}{l}\text { The PRIME-MD } \\
\text { Patient Health Questionnaire (PHQ) }\end{array}$} & \multirow[t]{3}{*}{27.37} & Total (6943) & $704(10.1 \%)$ & \multirow[t]{3}{*}{7} \\
\hline & & & & & & Male (6427) & $646(10.0 \%)$ & \\
\hline & & & & & & $\begin{array}{l}\text { Female } \\
(516)\end{array}$ & $58(46.7 \%)$ & \\
\hline \multirow{3}{*}{$\begin{array}{l}\text { Maguen, S. } \\
\text { et al. } \\
(2010)(116)\end{array}$} & \multirow[t]{3}{*}{ USA } & \multirow{3}{*}{$\begin{array}{l}\text { Convenience } \\
\text { Sampling } \\
\text { (CS) }\end{array}$} & \multirow{3}{*}{$\begin{array}{l}\text { Iraq and Afghanistan } \\
\text { Veterans Enrolled in } \\
\text { Veterans Affairs } \\
\text { Health Care }\end{array}$} & \multirow[t]{3}{*}{$\begin{array}{l}\text { Diagnostic and Statistical Manual of } \\
\text { Mental Disorders-IV (DSM-IV) }\end{array}$} & 31.21 & $\begin{array}{l}\text { Total } \\
\text { (329049) }\end{array}$ & $\begin{array}{l}57,051 \\
(17.33 \%)\end{array}$ & \multirow[t]{3}{*}{8} \\
\hline & & & & & 31.47 & $\begin{array}{l}\text { Male } \\
(288348)\end{array}$ & $47,876(17 \%)$ & \\
\hline & & & & & 29.41 & $\begin{array}{l}\text { Female } \\
(40701)\end{array}$ & $9175(23 \%)$ & \\
\hline \multirow{2}{*}{$\begin{array}{l}\text { Stecker, T. } \\
\text { et al. } \\
(2010)(117)\end{array}$} & \multirow[t]{2}{*}{ Lebanon } & \multirow{2}{*}{$\begin{array}{l}\text { Convenience } \\
\text { Sampling } \\
\text { (CS) }\end{array}$} & $\begin{array}{l}\text { Iraq/Afghanistan } \\
\text { veterans }\end{array}$ & \multirow[t]{2}{*}{$\begin{array}{l}\text { Diagnostic and Statistical Manual of } \\
\text { Mental Disorders-IV (DSM-IV) }\end{array}$} & \multirow[t]{2}{*}{34.4} & 293,861 & $\begin{array}{l}36,900 \\
(12.5 \%)\end{array}$ & \multirow[t]{2}{*}{6} \\
\hline & & & $\begin{array}{l}\text { Iraq/Afghanistan } \\
\text { Veterans with } \\
\text { Alcohol Use Disorder }\end{array}$ & & & 118,332 & $4568(3.8 \%)$ & \\
\hline \multirow{2}{*}{$\begin{array}{l}\text { Burnett- } \\
\text { Zeigler, l. } \\
\text { et al. } \\
(2011)(118)\end{array}$} & \multirow[t]{2}{*}{ USA } & \multirow{2}{*}{$\begin{array}{l}\text { Random } \\
\text { Sampling } \\
\text { (CS) }\end{array}$} & $\begin{array}{l}\text { Afghanistan and Iraq } \\
\text { Veterans }\end{array}$ & \multirow[t]{2}{*}{$\begin{array}{l}\text { The PRIME-MD } \\
\text { Patient Health Questionnaire (PHQ) }\end{array}$} & \multirow[t]{2}{*}{-} & 362 & $64(17.6 \%)$ & \multirow[t]{2}{*}{7} \\
\hline & & & $\begin{array}{l}\text { Iraq/Afghanistan } \\
\text { Veterans with } \\
\text { Alcohol Use Disorder }\end{array}$ & & & 200 & $72(36 \%)$ & \\
\hline $\begin{array}{l}\text { Erbes, C. R. } \\
\text { et al. } \\
(2011)(119)\end{array}$ & USA & $\begin{array}{l}\text { Convenience } \\
\text { Sampling } \\
\text { (CS) }\end{array}$ & $\begin{array}{l}\text { National Guard/ } \\
\text { Reserve veterans } \\
\text { returning from Iraq }\end{array}$ & The Beck Depression Inventory (BDI) & 31.60 & 617 & $83(13.5 \%)$ & 7 \\
\hline $\begin{array}{l}\text { Guerra, V. S. } \\
\text { et al. } \\
(2011)(120)\end{array}$ & USA & $\begin{array}{l}\text { Convenience } \\
\text { Sampling } \\
\text { (CS) }\end{array}$ & $\begin{array}{l}\text { Veterans in } \\
\text { Operations Enduring } \\
\text { Freedom and Iraqi } \\
\text { Freedom (OEF/OIF) }\end{array}$ & $\begin{array}{l}\text { The Beck Depression Inventory (BDI) } \\
\text { Beck Scale for Suicide Ideation Scale } \\
\text { for Suicide Ideation-Adapted }\end{array}$ & 38.3 & 393 & $88(22.4 \%)$ & 8 \\
\hline $\begin{array}{l}\text { Jakupcak, M. } \\
\text { et al. } \\
(2011)(121)\end{array}$ & USA & $\begin{array}{l}\text { Convenience } \\
\text { Sampling } \\
\text { (CS) }\end{array}$ & $\begin{array}{l}\text { Iraq and Afghanistan } \\
\text { War Veterans in the } \\
\text { U.S }\end{array}$ & $\begin{array}{l}\text { The PRIME-MD } \\
\text { Patient Health Questionnaire (PHQ) }\end{array}$ & 31 & 336 & $126(37.5 \%)$ & 7 \\
\hline Kehle, S. M. & USA & Convenience & Soldiers from a & Diagnostic and Statistical Manual of & 31.30 & Total (348) & $51(15 \%)$ & 7 \\
\hline $\begin{array}{l}\text { et al. } \\
(2011)(122)\end{array}$ & & $\begin{array}{l}\text { Sampling } \\
\text { (CS) }\end{array}$ & $\begin{array}{l}\text { Natıonal Guard } \\
\text { Brigade Combat }\end{array}$ & N & & Male (304) & $39(13 \%)$ & \\
\hline & & & Team (BCT) & & & Female (44) & $12(27 \%)$ & \\
\hline & & & $\begin{array}{l}\text { Alcohol use } \\
\text { disorders }\end{array}$ & & & 348 & 45 (13\%) & \\
\hline & & & $\begin{array}{l}\text { Substance use } \\
\text { disorders }\end{array}$ & & & 348 & $4(1 \%)$ & \\
\hline $\begin{array}{l}\text { Garber, B. G. } \\
\text { et al. } \\
(2012)(123)\end{array}$ & Canada & $\begin{array}{l}\text { Convenience } \\
\text { Sampling } \\
\text { (CS) }\end{array}$ & $\begin{array}{l}\text { Canadian Forces } \\
\text { Members While on } \\
\text { Deployment to } \\
\text { Afghanistan }\end{array}$ & $\begin{array}{l}\text { The PRIME-MD } \\
\text { Patient Health Questionnaire (PHQ) }\end{array}$ & - & 1572 & 73 (4.7\%) & 5 \\
\hline $\begin{array}{l}\text { Maguen, S. } \\
\text { et al. }\end{array}$ & USA & $\begin{array}{l}\text { Convenience } \\
\text { Sampling }\end{array}$ & $\begin{array}{l}\text { Iraq and Afghanistan } \\
\text { Veterans }\end{array}$ & $\begin{array}{l}\text { The Diagnostic and Statistical Manual- } \\
\text { Fourth Edition (DSM-IV) }\end{array}$ & 45 & $\begin{array}{l}\text { Total } \\
(74493)\end{array}$ & 41,424 (56\%) & 7 \\
\hline$(20 \mid 2)(124)$ & & & & & & $\begin{array}{l}\text { Male } \\
(67238)\end{array}$ & $36,359(54 \%)$ & \\
\hline & & & & & & Female & 5065 (70\%) & \\
\hline
\end{tabular}


Table 1 The study characteristics of included studies about depression (Continued)

\begin{tabular}{|c|c|c|c|c|c|c|c|c|}
\hline $\begin{array}{l}\text { Authors } \\
\text { (Years) }\end{array}$ & Country & $\begin{array}{l}\text { Type of } \\
\text { Sampling } \\
\text { (Type of } \\
\text { Study) }\end{array}$ & Study Population & Depression Assessment Method & $\begin{array}{l}\text { Age } \\
\text { (Mean) }\end{array}$ & $\begin{array}{l}\text { Sample } \\
\text { size }\end{array}$ & $\begin{array}{l}\text { Prevalence } \\
\text { of } \\
\text { Depression } \\
(\%)\end{array}$ & $\begin{array}{l}\text { NOS } \\
\text { Score }\end{array}$ \\
\hline & & & & & & $(7255)$ & & \\
\hline $\begin{array}{l}\text { Vasterling, J. } \\
\text { J. et al. } \\
\text { (2012)(125) }\end{array}$ & USA & $\begin{array}{l}\text { Convenience } \\
\text { Sampling } \\
\text { (CS) }\end{array}$ & $\begin{array}{l}\text { Iraq-deployed US } \\
\text { Army soldiers }\end{array}$ & $\begin{array}{l}\text { The Center for Epidemiological } \\
\text { Studies Depression Scale (CES-D) }\end{array}$ & 25.1 & 760 & $238(31.3 \%)$ & 7 \\
\hline $\begin{array}{l}\text { Cohen, S. I. } \\
\text { et al. } \\
\text { (2013)(126) }\end{array}$ & USA & $\begin{array}{l}\text { Convenience } \\
\text { Sampling } \\
\text { (CS) }\end{array}$ & $\begin{array}{l}\text { US military veterans } \\
\text { returning from Iraq } \\
\text { and Afghanistan }\end{array}$ & $\begin{array}{l}\text { The Diagnostic and Statistical Manual- } \\
\text { Fourth Edition (DSM-IV) }\end{array}$ & - & 93 & $44(47.3 \%)$ & 6 \\
\hline \multirow{3}{*}{$\begin{array}{l}\text { Harbertson, J. } \\
\text { et al. } \\
(2013)(127)\end{array}$} & \multirow[t]{3}{*}{ USA } & \multirow{3}{*}{$\begin{array}{l}\text { Convenience } \\
\text { Sampling } \\
\text { (CS) }\end{array}$} & \multirow{2}{*}{$\begin{array}{l}\text { Male Rwanda } \\
\text { Defense Forces } \\
\text { military personnel }\end{array}$} & \multirow{3}{*}{$\begin{array}{l}\text { The Center for Epidemiological } \\
\text { Studies Depression Scale (CES-D) }\end{array}$} & \multirow[t]{3}{*}{30.9} & 1238 & $232(22.1 \%)$ & \multirow[t]{3}{*}{7} \\
\hline & & & & & & 546 & $129(23.7 \%)$ & \\
\hline & & & Alcohol Use Disorder & & & & & \\
\hline \multirow{3}{*}{$\begin{array}{l}\text { Marshall, B. D. } \\
\text { et al. } \\
(2013)(128)\end{array}$} & \multirow[t]{3}{*}{ USA } & \multirow{3}{*}{$\begin{array}{l}\text { Convenience } \\
\text { Sampling } \\
\text { (CS) }\end{array}$} & Ohio Army National & \multirow{3}{*}{$\begin{array}{l}\text { The PRIME-MD } \\
\text { Patient Health Questionnaire (PHQ) }\end{array}$} & \multirow[t]{3}{*}{ - } & 2117 & $128(6 \%)$ & \multirow[t]{3}{*}{7} \\
\hline & & & Guard Soldiers & & & 142 & $17(12 \%)$ & \\
\hline & & & Soldiers with HIV & & & & & \\
\hline $\begin{array}{l}\text { Morrow, C. E. } \\
\text { et al. } \\
(2013)(129)\end{array}$ & USA & $\begin{array}{l}\text { Convenience } \\
\text { Sampling } \\
\text { (CS) }\end{array}$ & U.S. Air Force & $\begin{array}{l}\text { The PRIME-MD } \\
\text { Patient Health Questionnaire (PHQ) }\end{array}$ & 30.35 & 194 & $3(1.6 \%)$ & 5 \\
\hline $\begin{array}{l}\text { Swinkels, C. } \\
\text { M. et al. } \\
(2013)(130)\end{array}$ & UK & $\begin{array}{l}\text { Convenience } \\
\text { Sampling } \\
\text { (CS) }\end{array}$ & $\begin{array}{l}\text { U.S. Afghanistan/Iraq } \\
\text { Veterans }\end{array}$ & $\begin{array}{l}\text { Diagnostic and Statistical Manual of } \\
\text { Mental Disorders-IV (DSM-IV) }\end{array}$ & 37.40 & 1640 & $308(18.8 \%)$ & 6 \\
\hline $\begin{array}{l}\text { Chapman, P. } \\
\text { L. et al. } \\
\text { (2014)(131) }\end{array}$ & USA & $\begin{array}{l}\text { Convenience } \\
\text { Sampling } \\
\text { (CS) }\end{array}$ & $\begin{array}{l}\text { U.S. Army Combat } \\
\text { Medics }\end{array}$ & $\begin{array}{l}\text { The PRIME-MD } \\
\text { Patient HealthQuestionnaire (PHQ) }\end{array}$ & 43.54 & 543 & $73(13.4 \%)$ & 6 \\
\hline \multirow{3}{*}{$\begin{array}{l}\text { Clarke- } \\
\text { Walper, K. } \\
\text { et al. } \\
(2014)(132)\end{array}$} & \multirow[t]{3}{*}{ USA } & \multirow{3}{*}{$\begin{array}{l}\text { Convenience } \\
\text { Sampling } \\
\text { (CS) }\end{array}$} & \multirow{2}{*}{$\begin{array}{l}\text { Soldiers who } \\
\text { returned from Iraq } \\
\text { or Afghanistan }\end{array}$} & \multirow{3}{*}{$\begin{array}{l}\text { The PRIME-MD } \\
\text { Patient Health Questionnaire (PHQ) }\end{array}$} & \multirow[t]{3}{*}{ - } & 7849 & $611(8.1 \%)$ & \multirow[t]{3}{*}{7} \\
\hline & & & & & & 2328 & $304(13.1 \%)$ & \\
\hline & & & Alcohol use & & & & & \\
\hline \multirow{5}{*}{$\begin{array}{l}\text { Curry, J. F. } \\
\text { et al. } \\
(2014)(133)\end{array}$} & \multirow[t]{5}{*}{ USA } & \multirow{5}{*}{$\begin{array}{l}\text { Convenience } \\
\text { Sampling } \\
\text { (CS) }\end{array}$} & \multirow[t]{3}{*}{ Veterans } & \multirow{5}{*}{$\begin{array}{l}\text { Diagnostic and Statistical Manual of } \\
\text { Mental Disorders-IV (DSM-IV) }\end{array}$} & \multirow[t]{5}{*}{37.48} & Total (1700) & $652(38.4 \%)$ & \multirow[t]{5}{*}{7} \\
\hline & & & & & & Male (1354) & 491 (36.3\%) & \\
\hline & & & & & & $\begin{array}{l}\text { Female } \\
(346)\end{array}$ & $161(46.5 \%)$ & \\
\hline & & & $\begin{array}{l}\text { Veterans with } \\
\text { alcohol use }\end{array}$ & & & 623 & $72(11.6 \%)$ & \\
\hline & & & $\begin{array}{l}\text { Veterans with } \\
\text { substance use }\end{array}$ & & & 154 & $7(4.5 \%)$ & \\
\hline $\begin{array}{l}\text { Denneson, L. } \\
\text { M. et al. } \\
\text { (2014)(134) }\end{array}$ & USA & $\begin{array}{l}\text { Convenience } \\
\text { Sampling } \\
\text { (CS) }\end{array}$ & $\begin{array}{l}\text { Iraq and Afghanistan } \\
\text { Veterans }\end{array}$ & $\begin{array}{l}\text { The PRIME-MD } \\
\text { Patient Health } \\
\text { Questionnaire (PHQ) }\end{array}$ & - & 465 & 237 (51\%) & 7 \\
\hline $\begin{array}{l}\text { Don } \\
\text { Richardson, J. } \\
\text { et al. } \\
(2014)(135)\end{array}$ & Canada & $\begin{array}{l}\text { Convenience } \\
\text { Sampling } \\
\text { (CS) }\end{array}$ & $\begin{array}{l}\text { Canadian Forces } \\
\text { members and } \\
\text { veterans }\end{array}$ & $\begin{array}{l}\text { The PRIME-MD } \\
\text { Patient Health Questionnaire (PHQ) }\end{array}$ & - & 404 & $316(78.2 \%)$ & 7 \\
\hline $\begin{array}{l}\text { Garber, B. G. } \\
\text { et al. } \\
(2014)(136)\end{array}$ & Canada & $\begin{array}{l}\text { Random } \\
\text { Sampling } \\
\text { (CS) }\end{array}$ & $\begin{array}{l}\text { Canadian armed } \\
\text { forces personnel }\end{array}$ & $\begin{array}{l}\text { The PRIME-MD } \\
\text { Patient Health Questionnaire (PHQ) }\end{array}$ & - & 16,153 & $593(3.67 \%)$ & 8 \\
\hline $\begin{array}{l}\text { Heltemes, K. } \\
\text { J. et al. } \\
(2014)(137)\end{array}$ & USA & $\begin{array}{l}\text { Random } \\
\text { Sampling } \\
\text { (CS) }\end{array}$ & Injured veterans & $\begin{array}{l}\text { Diagnostic and Statistical Manual of } \\
\text { Mental Disorders-IV (DSM-IV) }\end{array}$ & 22.5 & 812 & $146(18 \%)$ & 6 \\
\hline $\begin{array}{l}\text { Lehavot, K. } \\
\text { et al. } \\
\text { (2014)(138) }\end{array}$ & USA & $\begin{array}{l}\text { Random } \\
\text { Sampling } \\
\text { (CS) }\end{array}$ & $\begin{array}{l}\text { Sexual Minority and } \\
\text { Heterosexual } \\
\text { Women Veterans }\end{array}$ & $\begin{array}{l}\text { The PRIME-MD } \\
\text { Patient Health Questionnaire (PHQ) }\end{array}$ & 48 & 697 & $260(37.3 \%)$ & 7 \\
\hline $\begin{array}{l}\text { Ramsawh, H. } \\
\text { J. et al. }\end{array}$ & USA & $\begin{array}{l}\text { Convenience } \\
\text { Sampling }\end{array}$ & $\begin{array}{l}\text { Active Duty Military } \\
\text { Personnel }\end{array}$ & $\begin{array}{l}\text { 10-item Center for Epidemiologic } \\
\text { Studies Depression Scale }\end{array}$ & 35 & 5461 & 1914 (35\%) & 8 \\
\hline
\end{tabular}


Table 1 The study characteristics of included studies about depression (Continued)

\begin{tabular}{|c|c|c|c|c|c|c|c|c|}
\hline $\begin{array}{l}\text { Authors } \\
\text { (Years) }\end{array}$ & Country & $\begin{array}{l}\text { Type of } \\
\text { Sampling } \\
\text { (Type of } \\
\text { Study) } \\
\end{array}$ & Study Population & Depression Assessment Method & $\begin{array}{l}\text { Age } \\
\text { (Mean) }\end{array}$ & $\begin{array}{l}\text { Sample } \\
\text { size }\end{array}$ & $\begin{array}{l}\text { Prevalence } \\
\text { of } \\
\text { Depression } \\
\text { (\%) } \\
\end{array}$ & $\begin{array}{l}\text { NOS } \\
\text { Score }\end{array}$ \\
\hline$(2014)(139)$ & & $(\mathrm{CS})$ & & & & & & \\
\hline $\begin{array}{l}\text { Bin Zubair, U. } \\
\text { et al. } \\
(2015)(140)\end{array}$ & Pakistan & $\begin{array}{l}\text { Random } \\
\text { Sampling } \\
\text { (CS) }\end{array}$ & $\begin{array}{l}\text { All military recruits } \\
\text { were men and } \\
\text { above the age of } 17 \\
\text { years. }\end{array}$ & The Beck Depression Inventory (BDI) & 20 & 313 & 159 (50.7\%) & 7 \\
\hline $\begin{array}{l}\text { Cleveland, S. } \\
\text { D. et al. } \\
\text { (2015)(141) }\end{array}$ & USA & $\begin{array}{l}\text { Convenience } \\
\text { Sampling } \\
\text { (CS) }\end{array}$ & $\begin{array}{l}\text { Veterans and Civilian } \\
\text { College Students }\end{array}$ & $\begin{array}{l}\text { Diagnostic and Statistical Manual of } \\
\text { Mental Disorders-IV (DSM-IV) }\end{array}$ & - & 26,969 & $\begin{array}{l}7982 \\
(30.17 \%)\end{array}$ & 8 \\
\hline $\begin{array}{l}\text { Foote, C. E. } \\
\text { et al. } \\
\text { (2015)(142) }\end{array}$ & USA & $\begin{array}{l}\text { Random } \\
\text { Sampling } \\
\text { (CS) }\end{array}$ & Vietnam veterans & $\begin{array}{l}\text { The PRIME-MD } \\
\text { Patient Health Questionnaire (PHQ) }\end{array}$ & - & 247 & $44(17.8 \%)$ & 7 \\
\hline \multirow{3}{*}{$\begin{array}{l}\text { Hamilton, A. } \\
\text { B. et al. } \\
\text { (2015) (143) }\end{array}$} & \multirow[t]{3}{*}{ USA } & \multirow{3}{*}{$\begin{array}{l}\text { Random } \\
\text { Sampling } \\
\text { (CS) }\end{array}$} & Employed Women & \multirow{3}{*}{$\begin{array}{l}\text { The five-question Mental Health In- } \\
\text { ventory (MHI-5) }\end{array}$} & \multirow[t]{3}{*}{-} & 1410 & $120(4.1 \%)$ & 7 \\
\hline & & & veterans & & & \multirow[t]{2}{*}{195} & \multirow[t]{2}{*}{$42(27.3 \%)$} & \\
\hline & & & $\begin{array}{l}\text { Unemployed } \\
\text { Women Veterans }\end{array}$ & & & & & \\
\hline $\begin{array}{l}\text { Hoerster, K. } \\
\text { D. et al. } \\
\text { (2015)(144) }\end{array}$ & USA & $\begin{array}{l}\text { Random } \\
\text { Sampling } \\
\text { (CS) }\end{array}$ & $\begin{array}{l}\text { Iraq and Afghanistan } \\
\text { veterans }\end{array}$ & $\begin{array}{l}\text { The PRIME-MD } \\
\text { Patient Health Questionnaire (PHQ) }\end{array}$ & 31.3 & 332 & $53(16.3 \%)$ & 7 \\
\hline $\begin{array}{l}\text { Kim, N. Y. } \\
\text { et al. (2015) } \\
\text { (145) }\end{array}$ & USA & $\begin{array}{l}\text { Convenience } \\
\text { Sampling } \\
\text { (CS) }\end{array}$ & Korean Soldiers & $\begin{array}{l}\text { Scale for suicide ideation (SSI), The } \\
\text { Beck Depression Inventory (BDI) }\end{array}$ & 21.3 & 414 & $21(5 \%)$ & 6 \\
\hline $\begin{array}{l}\text { Lundin, A. } \\
\text { et al. } \\
\text { (2015)(146) }\end{array}$ & Sweden & $\begin{array}{l}\text { Random } \\
\text { Sampling } \\
\text { (CS) }\end{array}$ & Vietnam veterans & $\begin{array}{l}\text { Diagnostic and Statistical Manual of } \\
\text { Mental Disorders-IV (DSM-IV) }\end{array}$ & - & 4251 & $1263(29.7 \%)$ & 7 \\
\hline \multirow{2}{*}{$\begin{array}{l}\text { McGuire, A. } \\
\text { et al. } \\
(2015)(147)\end{array}$} & \multirow[t]{2}{*}{ UK } & \multirow{2}{*}{$\begin{array}{l}\text { Random } \\
\text { Sampling } \\
\text { (CS) }\end{array}$} & $\begin{array}{l}\text { Australian Defense } \\
\text { Force (ADF men) }\end{array}$ & \multirow[t]{2}{*}{$\begin{array}{l}\text { Diagnostic and Statistical Manual of } \\
\text { Mental Disorders-IV (DSM-IV) }\end{array}$} & \multirow[t]{2}{*}{50} & 4091 & $454(11 \%)$ & 7 \\
\hline & & & $\begin{array}{l}\text { Department of } \\
\text { Veterans' Affairs } \\
\text { (DVA women) }\end{array}$ & & & 4761 & 869 (18.3\%) & \\
\hline $\begin{array}{l}\text { Mysliwiec, V. } \\
\text { et al. } \\
(2015)(148)\end{array}$ & USA & $\begin{array}{l}\text { Convenience } \\
\text { Sampling } \\
\text { (CS) }\end{array}$ & $\begin{array}{l}\text { U.S. Military } \\
\text { Personnel }\end{array}$ & $\begin{array}{l}\text { Quick Inventory of Depressive } \\
\text { Symptomatology (QIDS) }\end{array}$ & 36.2 & 58 & $30(51.7 \%)$ & 7 \\
\hline \multirow{3}{*}{$\begin{array}{l}\text { Nasioudis, D. } \\
\text { et al. } \\
(2015)(149)\end{array}$} & \multirow[t]{3}{*}{ Greece } & \multirow{3}{*}{$\begin{array}{l}\text { Random } \\
\text { Sampling } \\
\text { (CS) }\end{array}$} & \multirow{3}{*}{$\begin{array}{l}\text { Greek military } \\
\text { medicine cadets }\end{array}$} & \multirow{3}{*}{$\begin{array}{l}\text { The Zung Self-report Depression Scale } \\
\text { (SDS) }\end{array}$} & \multirow[t]{3}{*}{19.84} & Total (146) & $57(39 \%)$ & \multirow[t]{3}{*}{7} \\
\hline & & & & & & Male (91) & $36(39.5 \%)$ & \\
\hline & & & & & & Female (55) & $21(38.2 \%)$ & \\
\hline $\begin{array}{l}\text { Vanderploeg, } \\
\text { R. D. et al. } \\
\text { (2015) (150) }\end{array}$ & USA & $\begin{array}{l}\text { Convenience } \\
\text { Sampling } \\
\text { (CS) }\end{array}$ & $\begin{array}{l}\text { Florida National } \\
\text { Guard Members }\end{array}$ & $\begin{array}{l}\text { The PRIME-MD } \\
\text { Patient Health Questionnaire (PHQ) }\end{array}$ & - & 3098 & $63(2 \%)$ & 7 \\
\hline $\begin{array}{l}\text { Fink, D. S. } \\
\text { et al. (2016) } \\
\text { (151) }\end{array}$ & USA & $\begin{array}{l}\text { Convenience } \\
\text { Sampling } \\
\text { (CS) }\end{array}$ & $\begin{array}{l}\text { U.S. Army National } \\
\text { Guard soldiers }\end{array}$ & $\begin{array}{l}\text { Diagnostic and Statistical Manual of } \\
\text { Mental Disorders-IV (DSM-IV) }\end{array}$ & 44 & 671 & $154(23 \%)$ & 8 \\
\hline $\begin{array}{l}\text { Forbes, D. } \\
\text { et al. } \\
(2016)(152)\end{array}$ & Australia & $\begin{array}{l}\text { Convenience } \\
\text { Sampling } \\
\text { (CS) }\end{array}$ & $\begin{array}{l}\text { Australian } \\
\text { peacekeepers }\end{array}$ & $\begin{array}{l}\text { Diagnostic and Statistical Manual of } \\
\text { Mental Disorders-IV (DSM-IV) }\end{array}$ & 46.5 & 2050 & $201(9.8 \%)$ & 8 \\
\hline $\begin{array}{l}\text { Guloglu, B. } \\
\text { et al. } \\
(2016)(153)\end{array}$ & Turkey & $\begin{array}{l}\text { Convenience } \\
\text { Sampling } \\
\text { (CS) }\end{array}$ & $\begin{array}{l}\text { Turkish combat- } \\
\text { injurednon- } \\
\text { professional veterans }\end{array}$ & The Brief Symptom Inventory (BSI) & 40 & 336 & $55(16.4 \%)$ & 7 \\
\hline $\begin{array}{l}\text { Hardos, J. E. } \\
\text { et al. (2016) } \\
\text { (154) }\end{array}$ & USA & $\begin{array}{l}\text { Convenience } \\
\text { Sampling } \\
\text { (CS) }\end{array}$ & $\begin{array}{l}\text { Aircraft Maintenance } \\
\text { Workers }\end{array}$ & $\begin{array}{l}\text { The PRIME-MD } \\
\text { Patient Health Questionnaire (PHQ) }\end{array}$ & 29 & 4801 & $1042(21.7 \%)$ & 7 \\
\hline \multirow{2}{*}{$\begin{array}{l}\text { Herberman } \\
\text { Mash, H. B. } \\
\text { et al. } \\
(2016)(155)\end{array}$} & \multirow[t]{2}{*}{ USA } & \multirow{2}{*}{$\begin{array}{l}\text { Convenience } \\
\text { Sampling } \\
\text { (CS) }\end{array}$} & U.S. Army soldiers & \multirow{2}{*}{$\begin{array}{l}\text { The 10-item Center for Epidemiologic } \\
\text { Studies Depression Scale }\end{array}$} & \multirow[t]{2}{*}{ - } & 3813 & $1368(35.8 \%)$ & \multirow[t]{2}{*}{8} \\
\hline & & & $\begin{array}{l}\text { U.S. Army soldiers } \\
\text { with alcohol use }\end{array}$ & & & 1210 & $583(48.18 \%)$ & \\
\hline
\end{tabular}


Table 1 The study characteristics of included studies about depression (Continued)

\begin{tabular}{|c|c|c|c|c|c|c|c|c|}
\hline $\begin{array}{l}\text { Authors } \\
\text { (Years) }\end{array}$ & Country & $\begin{array}{l}\text { Type of } \\
\text { Sampling } \\
\text { (Type of } \\
\text { Study) }\end{array}$ & Study Population & Depression Assessment Method & $\begin{array}{l}\text { Age } \\
\text { (Mean) }\end{array}$ & $\begin{array}{l}\text { Sample } \\
\text { size }\end{array}$ & $\begin{array}{l}\text { Prevalence } \\
\text { of } \\
\text { Depression } \\
\text { (\%) }\end{array}$ & $\begin{array}{l}\text { NOS } \\
\text { Score }\end{array}$ \\
\hline \multirow{3}{*}{$\begin{array}{l}\text { Monteith, L. } \\
\text { L. et al. } \\
(2016)(156)\end{array}$} & \multirow[t]{3}{*}{ USA } & \multirow{3}{*}{$\begin{array}{l}\text { Convenience } \\
\text { Sampling } \\
\text { (CS) }\end{array}$} & \multirow[t]{3}{*}{ Veterans } & \multirow{3}{*}{$\begin{array}{l}\text { Beck Scale for Suicide Ideation (BSS), } \\
\text { Multidimensional Suicide Inventory-28 } \\
\text { (MSI) Negative Affect scale }\end{array}$} & \multirow[t]{3}{*}{49.6} & Total (354) & 169 (47.7\%) & \multirow[t]{3}{*}{8} \\
\hline & & & & & & Male (310) & $146(47.1 \%)$ & \\
\hline & & & & & & Female (44) & $32(52.3 \%)$ & \\
\hline $\begin{array}{l}\text { Phillips, K. M. } \\
\text { et al. } \\
(2016)(157)\end{array}$ & USA & $\begin{array}{l}\text { Convenience } \\
\text { Sampling } \\
\text { (CS) }\end{array}$ & $\begin{array}{l}\text { Iraq- and } \\
\text { Afghanistan-era } \\
\text { Veterans }\end{array}$ & $\begin{array}{l}\text { 20-item, self-report Center for Epi- } \\
\text { demiological Studies Depression Scale } \\
\text { (CES-D) }\end{array}$ & 35.1 & 359 & $108(30 \%)$ & 6 \\
\hline $\begin{array}{l}\text { Zamorski, M. } \\
\text { A. et al. } \\
(2016)(158)\end{array}$ & Canada & $\begin{array}{l}\text { Convenience } \\
\text { Sampling } \\
\text { (CS) }\end{array}$ & $\begin{array}{l}\text { Canadian Armed } \\
\text { Forces }\end{array}$ & $\begin{array}{l}\text { Diagnostic and Statistical Manual of } \\
\text { Mental Disorders-IV (DSM-IV) }\end{array}$ & - & 5120 & $410(8 \%)$ & 7 \\
\hline \multirow{2}{*}{$\begin{array}{l}\text { Boakye, E. A. } \\
\text { et al. } \\
(2017)(159)\end{array}$} & \multirow[t]{2}{*}{ USA } & \multirow{2}{*}{$\begin{array}{l}\text { Random } \\
\text { Sampling } \\
\text { (CS) }\end{array}$} & Veterans & \multirow[t]{2}{*}{ Self-Report } & \multirow[t]{2}{*}{40} & 144 & $48(33.3 \%)$ & \multirow[t]{2}{*}{7} \\
\hline & & & $\begin{array}{l}\text { Veterans with } \\
\text { alcohol use }\end{array}$ & & & 75 & $24(32 \%)$ & \\
\hline \multirow{2}{*}{$\begin{array}{l}\text { Cohen, G. H. } \\
\text { et al. } \\
(2017)(160)\end{array}$} & \multirow[t]{2}{*}{ USA } & \multirow{2}{*}{$\begin{array}{l}\text { Convenience } \\
\text { Sampling } \\
\text { (CS) }\end{array}$} & $\begin{array}{l}\text { Army National Guard } \\
\text { Soldiers }\end{array}$ & \multirow{2}{*}{$\begin{array}{l}\text { The PRIME-MD } \\
\text { Patient Health Questionnaire (PHQ), } \\
\text { The PHQ-9 Item }\end{array}$} & \multirow[t]{2}{*}{ - } & 1582 & 164 (10.3\%) & \multirow[t]{2}{*}{8} \\
\hline & & & $\begin{array}{l}\text { Army National with } \\
\text { Alcohol Use }\end{array}$ & & & 93 & $27(29 \%)$ & \\
\hline \multirow{3}{*}{$\begin{array}{l}\text { Gradus, J. L. } \\
\text { et al. } \\
(2017)(161)\end{array}$} & \multirow[t]{3}{*}{ USA } & \multirow{3}{*}{$\begin{array}{l}\text { Random } \\
\text { Sampling } \\
\text { (CS) }\end{array}$} & \multirow{3}{*}{$\begin{array}{l}\text { Veterans of the Iraq } \\
\text { and Afghanistan } \\
\text { Wars }\end{array}$} & \multirow{3}{*}{$\begin{array}{l}\text { 20-item, self-report Center for Epi- } \\
\text { demiological Studies Depression Scale } \\
\text { (CES-D), The 4-item Suicidal Behaviors } \\
\text { Questionnaire-Short Form (SBQ-SF) }\end{array}$} & \multirow[t]{3}{*}{34} & Total (2244) & 712 (31.7\%) & \multirow[t]{3}{*}{7} \\
\hline & & & & & & Male (1062) & 314 (29.5\%) & \\
\hline & & & & & & $\begin{array}{l}\text { Female } \\
(1099)\end{array}$ & 398 (36.3\%) & \\
\hline \multirow{4}{*}{$\begin{array}{l}\text { Packnett, E. R. } \\
\text { et al. } \\
(2017)(162)\end{array}$} & \multirow[t]{4}{*}{ USA } & \multirow{4}{*}{$\begin{array}{l}\text { Convenience } \\
\text { Sampling } \\
\text { (CS) }\end{array}$} & Army & \multirow{4}{*}{$\begin{array}{l}\text { Diagnostic and Statistical Manual of } \\
\text { Mental Disorders-IV (DSM-IV) }\end{array}$} & \multirow[t]{4}{*}{ - } & 34,487 & 1777 (5.1\%) & \multirow[t]{4}{*}{8} \\
\hline & & & Navy & & & 6602 & $263(4 \%)$ & \\
\hline & & & Marine Corps & & & 8428 & $113(1.3 \%)$ & \\
\hline & & & Air Force & & & 9510 & $729(7.6 \%)$ & \\
\hline $\begin{array}{l}\text { Weeks, M. } \\
\text { et al. } \\
(2017)(163)\end{array}$ & Canada & $\begin{array}{l}\text { Convenience } \\
\text { Sampling } \\
\text { (CS) }\end{array}$ & $\begin{array}{l}\text { Canadian Military } \\
\text { and Civilian } \\
\text { Populations }\end{array}$ & $\begin{array}{l}\text { Diagnostic and Statistical Manual of } \\
\text { Mental Disorders-IV (DSM-IV) }\end{array}$ & 35 & 6696 & $536(8 \%)$ & 8 \\
\hline $\begin{array}{l}\text { Bartlett, B. A. } \\
\text { et al. } \\
(2018)(164)\end{array}$ & USA & $\begin{array}{l}\text { Convenience } \\
\text { Sampling } \\
\text { (CS) }\end{array}$ & Military veterans & $\begin{array}{l}\text { 20-item, self-report Center for Epi- } \\
\text { demiological Studies Depression Scale } \\
\text { (CES-D) }\end{array}$ & 38.40 & 910 & 75 (9.8\%) & 6 \\
\hline $\begin{array}{l}\text { Blakey, S. M. } \\
\text { et al. } \\
(2018)(165)\end{array}$ & USA & $\begin{array}{l}\text { Convenience } \\
\text { Sampling } \\
\text { (CS) }\end{array}$ & $\begin{array}{l}\text { U.S. veterans, active } \\
\text { duty personnel, and } \\
\text { National Guard and } \\
\text { Reserve members }\end{array}$ & $\begin{array}{l}\text { Diagnostic and Statistical Manual of } \\
\text { Mental Disorders-IV (DSM-IV) }\end{array}$ & 37.8 & 667 & 169 (25.3\%) & 7 \\
\hline $\begin{array}{l}\text { Boulos, D. } \\
\text { et al. }\end{array}$ & Canada & $\begin{array}{l}\text { Random } \\
\text { Sampling }\end{array}$ & $\begin{array}{l}\text { Regular Force } \\
\text { personnel }\end{array}$ & $\begin{array}{l}\text { Diagnostic and Statistical Manual of } \\
\text { Mental Disorders-IV (DSM-IV) }\end{array}$ & - & 3385 & $129(3.8 \%)$ & 7 \\
\hline$(2018)(166)$ & & & $\begin{array}{l}\text { Reserve Force } \\
\text { personnel }\end{array}$ & & & 1469 & 55 (3.7\%) & \\
\hline $\begin{array}{l}\text { Dillon, K. H. } \\
\text { et al. } \\
(2018)(167)\end{array}$ & USA & $\begin{array}{l}\text { Convenience } \\
\text { Sampling } \\
\text { (CS) }\end{array}$ & $\begin{array}{l}\text { Iraq/Afghanistan-era } \\
\text { veterans }\end{array}$ & $\begin{array}{l}\text { The Beck Scale for Suicide Ideation } \\
\text { (BSS), The Structured Clinical Interview } \\
\text { for DSM-IV-TR (SCID) }\end{array}$ & - & 3238 & $1315(40.6 \%)$ & 7 \\
\hline $\begin{array}{l}\text { Don } \\
\text { Richardson, J. } \\
\text { et al. } \\
(2018)(168)\end{array}$ & Canada & $\begin{array}{l}\text { Convenience } \\
\text { Sampling } \\
\text { (CS) }\end{array}$ & $\begin{array}{l}\text { Canadian Armed } \\
\text { Forces members } \\
\text { and veterans }\end{array}$ & $\begin{array}{l}\text { The PRIME-MD } \\
\text { Patient Health Questionnaire (PHQ) }\end{array}$ & 44.6 & 522 & $413(79.1 \%)$ & 7 \\
\hline $\begin{array}{l}\text { Elbogen, E. B. } \\
\text { et al. } \\
(2018)(169)\end{array}$ & USA & $\begin{array}{l}\text { Convenience } \\
\text { Sampling } \\
\text { (CS) }\end{array}$ & $\begin{array}{l}\text { Iraq/Afghanistan-era } \\
\text { veterans }\end{array}$ & $\begin{array}{l}\text { Diagnostic and Statistical Manual of } \\
\text { Mental Disorders-IV (DSM-IV) }\end{array}$ & 34.9 & 1172 & 375 (32\%) & 6 \\
\hline $\begin{array}{l}\text { Hourani, L. L. } \\
\text { et al. } \\
\text { (2018)(170) }\end{array}$ & USA & $\begin{array}{l}\text { Convenience } \\
\text { Sampling } \\
\text { (CS) }\end{array}$ & $\begin{array}{l}\text { Active duty military } \\
\text { personnel }\end{array}$ & $\begin{array}{l}\text { The PRIME-MD } \\
\text { Patient Health Questionnaire (PHQ), } \\
\text { Checklist }\end{array}$ & - & 947 & 115 (15.4\%) & 7 \\
\hline
\end{tabular}


Table 1 The study characteristics of included studies about depression (Continued)

\begin{tabular}{|c|c|c|c|c|c|c|c|c|}
\hline $\begin{array}{l}\text { Authors } \\
\text { (Years) }\end{array}$ & Country & $\begin{array}{l}\text { Type of } \\
\text { Sampling } \\
\text { (Type of } \\
\text { Study) }\end{array}$ & Study Population & Depression Assessment Method & $\begin{array}{l}\text { Age } \\
\text { (Mean) }\end{array}$ & $\begin{array}{l}\text { Sample } \\
\text { size }\end{array}$ & $\begin{array}{l}\text { Prevalence } \\
\text { of } \\
\text { Depression } \\
(\%)\end{array}$ & $\begin{array}{l}\text { NOS } \\
\text { Score }\end{array}$ \\
\hline $\begin{array}{l}\text { Kizilhan, J. I. } \\
\text { et al. } \\
(2018)(171)\end{array}$ & Iraq & $\begin{array}{l}\text { Convenience } \\
\text { Sampling } \\
\text { (CS) }\end{array}$ & Child soldiers & $\begin{array}{l}\text { Diagnostic and Statistical Manual of } \\
\text { Mental Disorders-IV (DSM-IV) }\end{array}$ & 12.6 & 81 & $37(45.6 \%)$ & 6 \\
\hline $\begin{array}{l}\text { McDonald, S. } \\
\text { D. et al. } \\
(2018)(172)\end{array}$ & USA & $\begin{array}{l}\text { Convenience } \\
\text { Sampling } \\
\text { (CS) }\end{array}$ & $\begin{array}{l}\text { U.S. Department of } \\
\text { Veterans Affairs } \\
\text { outpatients }\end{array}$ & $\begin{array}{l}\text { Diagnostic and Statistical Manual of } \\
\text { Mental Disorders-IV (DSM-IV) }\end{array}$ & 58.1 & 280 & $53(19 \%)$ & 7 \\
\hline $\begin{array}{l}\text { Stefanovics, E. } \\
\text { A. et al. } \\
(2018)(173)\end{array}$ & USA & $\begin{array}{l}\text { Convenience } \\
\text { Sampling } \\
\text { (CS) }\end{array}$ & US Veterans & The Patient Health Questionnaire-4 & 59 & 3122 & $209(6.7 \%)$ & 7 \\
\hline $\begin{array}{l}\text { Vun, E. et al. } \\
\text { (2018)(174) }\end{array}$ & Canada & $\begin{array}{l}\text { Convenience } \\
\text { Sampling } \\
\text { (CS) }\end{array}$ & $\begin{array}{l}\text { Canadian Armed } \\
\text { Forces active } \\
\text { personnel }\end{array}$ & $\begin{array}{l}\text { Diagnostic and Statistical Manual of } \\
\text { Mental Disorders-IV } \\
\text { (DSM-IV) }\end{array}$ & 35.4 & 6696 & $517(8 \%)$ & 8 \\
\hline $\begin{array}{l}\text { Waitzkin, } \mathrm{H} \text {. } \\
\text { et al. } \\
(2018)(175)\end{array}$ & USA & $\begin{array}{l}\text { Convenience } \\
\text { Sampling } \\
\text { (CS) }\end{array}$ & Military Personnel & $\begin{array}{l}\text { The PRIME-MD } \\
\text { Patient Health } \\
\text { Questionnaire } \\
\text { (PHQ) }\end{array}$ & - & 198 & $143(72 \%)$ & 7 \\
\hline $\begin{array}{l}\text { Byrne, S. P. } \\
\text { et al. } \\
(2019)(176)\end{array}$ & USA & $\begin{array}{l}\text { Convenience } \\
\text { Sampling } \\
\text { (CS) }\end{array}$ & U.S. military veterans & $\begin{array}{l}\text { The PRIME-MD } \\
\text { Patient Health } \\
\text { Questionnaire } \\
\text { (PHQ) }\end{array}$ & 53.4 & 158 & $62(34.7 \%)$ & 7 \\
\hline $\begin{array}{l}\text { Carney, B. } \\
\text { et al. } \\
(2019)(177)\end{array}$ & USA & $\begin{array}{l}\text { Random } \\
\text { Sampling } \\
\text { (CS) }\end{array}$ & $\begin{array}{l}\text { US Military } \\
\text { population with HIV } \\
\text { infection }\end{array}$ & $\begin{array}{l}\text { 20- } \\
\text { item, self-report Center for Epidemio- } \\
\text { logical Studies Depression Scale (CES- } \\
\text { D) }\end{array}$ & 32 & 662 & $114(17.2 \%)$ & 8 \\
\hline \multirow{3}{*}{$\begin{array}{l}\text { Jones, N. } \\
\text { et al. } \\
(2019)(178)\end{array}$} & \multirow[t]{3}{*}{ UK } & \multirow{3}{*}{$\begin{array}{l}\text { Random } \\
\text { Sampling } \\
\text { (CS) }\end{array}$} & \multirow[t]{3}{*}{ UK Armed Forces } & \multirow{3}{*}{$\begin{array}{l}\text { The PRIME-MD } \\
\text { Patient Health } \\
\text { Questionnaire } \\
\text { (PHQ) }\end{array}$} & \multirow[t]{3}{*}{-} & Total (1448) & $110(7.6 \%)$ & \multirow[t]{3}{*}{6} \\
\hline & & & & & & Male (1229) & $93(7.7 \%)$ & \\
\hline & & & & & & $\begin{array}{l}\text { Female } \\
(219)\end{array}$ & $17(7.9 \%)$ & \\
\hline \multirow{3}{*}{$\begin{array}{l}\text { Lucas, C. L. } \\
\text { et al. } \\
(2019)(179)\end{array}$} & \multirow[t]{3}{*}{ USA } & \multirow{3}{*}{$\begin{array}{l}\text { Convenience } \\
\text { Sampling } \\
\text { (CS) }\end{array}$} & \multirow[t]{3}{*}{ Military Personnel } & \multirow{3}{*}{$\begin{array}{l}\text { The PRIME-MD } \\
\text { Patient Health } \\
\text { Questionnaire } \\
\text { (PHQ) }\end{array}$} & \multirow[t]{3}{*}{-} & Total (1980) & $660(37.9 \%)$ & \multirow[t]{3}{*}{7} \\
\hline & & & & & & Male (1665) & $530(36.2 \%)$ & \\
\hline & & & & & & $\begin{array}{l}\text { Female } \\
(315)\end{array}$ & $130(46.8 \%)$ & \\
\hline $\begin{array}{l}\text { Nichter, B. } \\
\text { et al. } \\
(2019)(180)\end{array}$ & USA & $\begin{array}{l}\text { Random } \\
\text { Sampling } \\
\text { (CS) }\end{array}$ & $\begin{array}{l}\text { U.S. } \\
\text { veteran population }\end{array}$ & $\begin{array}{l}\text { The Patient Health } \\
\text { Questionnaire-4 (PHQ-4), The Patient } \\
\text { Health } \\
\text { Questionnaire-9 (PHQ-9) }\end{array}$ & 60.3 & 2732 & $201(7.3 \%)$ & 9 \\
\hline $\begin{array}{l}\text { Start, A. R. } \\
\text { et al. } \\
\text { (2019)(181) }\end{array}$ & USA & $\begin{array}{l}\text { Convenience } \\
\text { Sampling } \\
\text { (CS) }\end{array}$ & Military Personnel & $\begin{array}{l}\text { The Patient Health } \\
\text { Questionnaire-9 (PHQ-9) }\end{array}$ & - & 944 & $72(7.6 \%)$ & 7 \\
\hline $\begin{array}{l}\text { Blosnich, J. R. } \\
\text { et al. } \\
\text { (2020)(182) }\end{array}$ & USA & $\begin{array}{l}\text { Random } \\
\text { Sampling } \\
\text { (CS) }\end{array}$ & Military Veterans & $\begin{array}{l}\text { Diagnostic and Statistical Manual of } \\
\text { Mental Disorders-IV } \\
\text { (DSM-IV) }\end{array}$ & - & 293,872 & $\begin{array}{l}45,391 \\
(15.4 \%)\end{array}$ & 9 \\
\hline $\begin{array}{l}\text { Forys- } \\
\text { Donahue, K. } \\
\text { L. et al. } \\
(2020)(183)\end{array}$ & USA & $\begin{array}{l}\text { Random } \\
\text { Sampling } \\
\text { (CS) }\end{array}$ & US Army population & $\begin{array}{l}\text { The Patient Health } \\
\text { Questionnaire-9 (PHQ-9) }\end{array}$ & - & 7043 & $774(11 \%)$ & 6 \\
\hline $\begin{array}{l}\text { Gjerstad, C. L. } \\
\text { et al. } \\
(2020)(184)\end{array}$ & Norway & $\begin{array}{l}\text { Convenience } \\
\text { Sampling } \\
\text { (CS) }\end{array}$ & $\begin{array}{l}\text { Norwegian } \\
\text { Peacekeepers }\end{array}$ & $\begin{array}{l}\text { The Hospital Anxiety and Depression } \\
\text { Scale (HADS) }\end{array}$ & 30 & 10,450 & $417(4 \%)$ & 8 \\
\hline $\begin{array}{l}\text { Groll, D. L. } \\
\text { et al. } \\
(2020)(185)\end{array}$ & Canada & $\begin{array}{l}\text { Convenience } \\
\text { Sampling } \\
\text { (CS) }\end{array}$ & $\begin{array}{l}\text { Canadian military } \\
\text { persons }\end{array}$ & $\begin{array}{l}\text { The Patient Health } \\
\text { Questionnaire-9 (PHQ-9) }\end{array}$ & - & 477 & $61(12.8 \%)$ & 8 \\
\hline \multirow{2}{*}{$\begin{array}{l}\text { Gross, G. M. } \\
\text { et al. } \\
(2020)(186)\end{array}$} & \multirow[t]{2}{*}{ USA } & \multirow{2}{*}{$\begin{array}{l}\text { Random } \\
\text { Sampling } \\
\text { (CS) }\end{array}$} & \multirow{2}{*}{$\begin{array}{l}\text { U.S. } \\
\text { veteran population }\end{array}$} & \multirow{2}{*}{$\begin{array}{l}\text { The Patient Health } \\
\text { Questionnaire-9 (PHQ-9) }\end{array}$} & \multirow[t]{2}{*}{35} & Total (650) & $306(47 \%)$ & \multirow[t]{2}{*}{7} \\
\hline & & & & & & Male (498) & 192 (38.6\%) & \\
\hline
\end{tabular}


Table 1 The study characteristics of included studies about depression (Continued)

\begin{tabular}{|c|c|c|c|c|c|c|c|c|}
\hline $\begin{array}{l}\text { Authors } \\
\text { (Years) }\end{array}$ & Country & $\begin{array}{l}\text { Type of } \\
\text { Sampling } \\
\text { (Type of } \\
\text { Study) }\end{array}$ & Study Population & Depression Assessment Method & $\begin{array}{l}\text { Age } \\
\text { (Mean) }\end{array}$ & $\begin{array}{l}\text { Sample } \\
\text { size }\end{array}$ & $\begin{array}{l}\text { Prevalence } \\
\text { of } \\
\text { Depression } \\
(\%)\end{array}$ & $\begin{array}{l}\text { NOS } \\
\text { Score }\end{array}$ \\
\hline & & & & & & $\begin{array}{l}\text { Female } \\
(352)\end{array}$ & $114(32.4 \%)$ & \\
\hline $\begin{array}{l}\text { Shim, E. J. } \\
\text { et al. } \\
(2020)(187)\end{array}$ & Korea & $\begin{array}{l}\text { Random } \\
\text { Sampling } \\
\text { (CS) }\end{array}$ & $\begin{array}{l}\text { Korean military } \\
\text { population }\end{array}$ & $\begin{array}{l}\text { The Mini } \\
\text { International Neuropsychiatric } \\
\text { Interview Plus (MINI-Plus), The Patient } \\
\text { Health } \\
\text { Questionnaire-9 (PHQ-9) }\end{array}$ & 50.6 & 1937 & $162(8.4 \%)$ & 8 \\
\hline $\begin{array}{l}\text { Smigelsky, M. } \\
\text { A. et al. } \\
(2020)(188)\end{array}$ & USA & $\begin{array}{l}\text { Convenience } \\
\text { Sampling } \\
\text { (CS) }\end{array}$ & $\begin{array}{l}\text { U.S. military } \\
\text { population }\end{array}$ & $\begin{array}{l}\text { Diagnostic and Statistical Manual of } \\
\text { Mental Disorders-IV } \\
\text { (DSM-IV) }\end{array}$ & 37.6 & 1002 & $210(21 \%)$ & 6 \\
\hline $\begin{array}{l}\text { Smith, L. M. } \\
\text { et al. } \\
(2020)(189)\end{array}$ & USA & $\begin{array}{l}\text { Convenience } \\
\text { Sampling } \\
\text { (CS) }\end{array}$ & $\begin{array}{l}\text { U.S. Air Force Basic } \\
\text { Military Training }\end{array}$ & $\begin{array}{l}\text { The Patient Health } \\
\text { Questionnaire-9 (PHQ-9) }\end{array}$ & - & 85 & $20(23.5 \%)$ & 5 \\
\hline $\begin{array}{l}\text { Stefanovics, E. } \\
\text { A. et al. } \\
(2020)(190)\end{array}$ & USA & $\begin{array}{l}\text { Convenience } \\
\text { Sampling } \\
\text { (CS) }\end{array}$ & U.S. Military Veterans & $\begin{array}{l}\text { The Mini International } \\
\text { Neuropsychiatric Interview (MINI), The } \\
\text { Patient Health } \\
\text { Questionnaire-9 (PHQ-9) }\end{array}$ & 55 & 1308 & $340(30 \%)$ & 5 \\
\hline $\begin{array}{l}\text { Taillieu, T. L. } \\
\text { et al. } \\
(2020)(191)\end{array}$ & Canada & $\begin{array}{l}\text { Convenience } \\
\text { Sampling } \\
\text { (CS) }\end{array}$ & $\begin{array}{l}\text { Canadian Armed } \\
\text { Forces }\end{array}$ & $\begin{array}{l}\text { Diagnostic and Statistical Manual of } \\
\text { Mental Disorders-IV } \\
\text { (DSM-IV) }\end{array}$ & - & 6447 & 1006(15.6\%) & 5 \\
\hline $\begin{array}{l}\text { Wang, J. et al. } \\
(2020)(192)\end{array}$ & USA & $\begin{array}{l}\text { Convenience } \\
\text { Sampling } \\
\text { (CS) }\end{array}$ & $\begin{array}{l}\text { U.S. } \\
\text { Reserve and } \\
\text { National Guard } \\
\text { Personnel }\end{array}$ & $\begin{array}{l}\text { The Patient Health } \\
\text { Questionnaire-9 (PHQ-9) }\end{array}$ & 34.4 & 3503 & $86(2.5 \%)$ & 6 \\
\hline $\begin{array}{l}\text { Ursano, R. J. } \\
\text { et al. } \\
(2020)(193)\end{array}$ & USA & $\begin{array}{l}\text { Convenience } \\
\text { Sampling } \\
\text { (CS) }\end{array}$ & $\begin{array}{l}\text { US Army Soldiers } \\
\text { During Deployment } \\
\text { in Afghanistan }\end{array}$ & $\begin{array}{l}\text { Diagnostic and Statistical Manual of } \\
\text { Mental Disorders-IV } \\
\text { (DSM-IV) }\end{array}$ & - & 3957 & $173(4.1 \%)$ & 7 \\
\hline $\begin{array}{l}\text { Yeom, C. W. } \\
\text { et al. } \\
(2020)(194)\end{array}$ & Korea & $\begin{array}{l}\text { Convenience } \\
\text { Sampling } \\
\text { (CS) }\end{array}$ & $\begin{array}{l}\text { Korean military } \\
\text { personal }\end{array}$ & $\begin{array}{l}\text { The Mini } \\
\text { International Neuropsychiatric } \\
\text { Interview Plus } \\
\text { (MINI-Plus Suicidality module), The } \\
\text { Patient Health } \\
\text { Questionnaire-9 (PHQ-9) }\end{array}$ & 21.4 & 480 & $27(5.6 \%)$ & 6 \\
\hline
\end{tabular}

women was 110,847 and 4717 people, respectively, of whom 50,370 and 893 were depressed, respectively. The results of meta-analysis showed that the pooled prevalence of depression in male soldiers was equal to $23 \%$ (\% $95 \mathrm{CI} ; 12-37 \%)$ while in military women, it was equal to 25\% (\% 95 CI; 31-40\%) (Table 3).

Thirty-six cross-sectional studies had used the diagnostic and statistical manual of mental disorders-IV (DSM-IV), 24 studies had used the patient health questionnaire (PHQ), 13 studies had applied interviews using clinical criteria and symptoms, 5 studies had applied the Zung self-tool report depression scale (SDS), 9 studies had used the beck depression inventory (BDI) and 7 studies had used the center for epidemiological studies depression (CES-D) to diagnose depression in the active or in-service military. The overall prevalence of depression according to the diagnostic and statistical manual of mental disorders-IV (DSM-IV) was 15\% (\% $95 \mathrm{CI}$;
17-35\%), according to the patient health questionnaire (PHQ), it was 15\% (\% $95 \mathrm{CI}$; 13-17\%), And according to the Zung self-report depression scale (SDS), it was equal to $20 \%$ (\% $95 \mathrm{CI} ; 14-26 \%$ ). Also, the overall pooled prevalence based on beck depression inventory (BDI) and the center for epidemiological studies depression (CES-D) was 25\% (\% $95 \mathrm{CI}$; 15-36\%) and 13\% (\% $95 \mathrm{CI}$; 8-19\%), respectively (Table 3 ).

In the case of the active military, subgroup results by country showed most studies had been conducted in the United States that after combining 66 studies conducted in this country, the prevalence of depression was $21 \%$ (with a confidence interval of 17 to $25 \%$ ). The prevalence of depression in Thailand and the UK, which was 39 and $30 \%$, respectively, was higher than that in other countries. The rest of the studies had been individually conducted in only one country and because of their number of primary studies not be used for meta-analysis (Table 3). 
Table 2 The study characteristics of included studies about suicide attempted and thought

\begin{tabular}{|c|c|c|c|c|c|c|c|c|c|}
\hline \multirow[t]{2}{*}{$\begin{array}{l}\text { Authors } \\
\text { (Years) }\end{array}$} & \multirow[t]{2}{*}{ Country } & \multirow{2}{*}{$\begin{array}{l}\text { Type of } \\
\text { Sampling } \\
\text { (Type of } \\
\text { Study) }\end{array}$} & \multirow[t]{2}{*}{$\begin{array}{l}\text { Study } \\
\text { Population }\end{array}$} & \multirow[t]{2}{*}{$\begin{array}{l}\text { Depression Assessment } \\
\text { Method }\end{array}$} & \multirow[t]{2}{*}{$\begin{array}{l}\text { Age } \\
\text { (Mean) }\end{array}$} & \multirow[t]{2}{*}{$\begin{array}{l}\text { Sample } \\
\text { size }\end{array}$} & \multicolumn{2}{|c|}{$\begin{array}{l}\text { Prevalence of } \\
\text { Suicide (\%) }\end{array}$} & \multirow[t]{2}{*}{$\begin{array}{l}\text { NOS } \\
\text { Score }\end{array}$} \\
\hline & & & & & & & Attempts & Thoughts & \\
\hline $\begin{array}{l}\text { Helzer, J. E. } \\
\text { et al. (1976) } \\
\text { (66) }\end{array}$ & USA & $\begin{array}{l}\text { Random } \\
\text { Sampling } \\
\text { (CS) }\end{array}$ & Army men & Clinical Symptoms (Interviews) & - & 470 & $N R$ & $42(9 \%)$ & 7 \\
\hline $\begin{array}{l}\text { Bohnker, B. } \\
\text { et al. (1992) } \\
\text { (195) }\end{array}$ & USA & $\begin{array}{l}\text { Random } \\
\text { Sampling } \\
\text { (CS) }\end{array}$ & $\begin{array}{l}\text { Aircraft Carrier } \\
\text { (men) }\end{array}$ & $N R$ & - & 150 & $102(68 \%)$ & NR & 6 \\
\hline $\begin{array}{l}\text { Brown, G. R. } \\
\text { et al. (1993) } \\
\text { (70) }\end{array}$ & USA & $\begin{array}{l}\text { Random } \\
\text { Sampling } \\
\text { (CS) }\end{array}$ & $\begin{array}{l}\text { Air Forces men } \\
\text { with HIV }\end{array}$ & $\begin{array}{l}\text { Structured Interview Guide for the } \\
\text { Hamilton } \\
\text { Anxiety and Depression Scales } \\
\text { (SIGH-AD) }\end{array}$ & 35 & 442 & $24(5.4 \%)$ & NR & 8 \\
\hline $\begin{array}{l}\text { Lish, J. D. et al. } \\
(1996)(74)\end{array}$ & USA & $\begin{array}{l}\text { Random } \\
\text { Sampling } \\
\text { (CS) }\end{array}$ & $\begin{array}{l}\text { Army men and } \\
\text { women }\end{array}$ & $\begin{array}{l}\text { Brief self-report questionnaire } \\
\text { (SCRENNER) }\end{array}$ & 21.2 & 669 & NR & $51(7.62 \%)$ & 7 \\
\hline $\begin{array}{l}\text { Benda, B. B. } \\
\text { (2003) (196) }\end{array}$ & USA & $\begin{array}{l}\text { Convenience } \\
\text { Sampling } \\
\text { (CS) }\end{array}$ & $\begin{array}{l}\text { Veterans } \\
\text { Who Abuse } \\
\text { Substances }\end{array}$ & $\begin{array}{l}\text { Multi-Problem Screening } \\
\text { Inventory (MPSI) }\end{array}$ & 50.3 & 600 & $240(40 \%)$ & $\begin{array}{l}184 \\
(30.7 \%)\end{array}$ & 7 \\
\hline $\begin{array}{l}\text { Ritchie, E. C. } \\
\text { et al. } \\
(2003)(197)\end{array}$ & USA & $\begin{array}{l}\text { Convenience } \\
\text { Sampling } \\
\text { (CS) }\end{array}$ & $\begin{array}{l}\text { Men and Women } \\
\text { in the Navy and } \\
\text { Marine Corps }\end{array}$ & - & 43 & 100 & $54(54 \%)$ & NR & 5 \\
\hline $\begin{array}{l}\text { Benda, B. B. } \\
\text { et al. } \\
(2005)(198)\end{array}$ & USA & $\begin{array}{l}\text { Convenience } \\
\text { Sampling } \\
\text { (CS) }\end{array}$ & $\begin{array}{l}\text { Veterans } \\
\text { Who Abuse } \\
\text { Substances }\end{array}$ & $\begin{array}{l}\text { The Multi-Problem Screening In- } \\
\text { ventory (MPSI) }\end{array}$ & 40.3 & 625 & $\begin{array}{l}197 \\
(31.5 \%)\end{array}$ & $\begin{array}{l}291 \\
(46.5 \%)\end{array}$ & 6 \\
\hline $\begin{array}{l}\text { Hoge, C. W. } \\
\text { et al. } \\
(2006)(101)\end{array}$ & USA & $\begin{array}{l}\text { Random } \\
\text { Sampling } \\
\text { (CS) }\end{array}$ & $\begin{array}{l}\text { Army soldiers and } \\
\text { Marines }\end{array}$ & $\begin{array}{l}\text { The PRIME-MD } \\
\text { Patient Health Questionnaire } \\
\text { (PHQ) }\end{array}$ & 31.2 & 303,905 & NR & $\begin{array}{l}3501 \\
(1.15 \%)\end{array}$ & 8 \\
\hline $\begin{array}{l}\text { Dove, M. B. } \\
\text { et al. } \\
(2007)(104)\end{array}$ & USA & $\begin{array}{l}\text { Convenience } \\
\text { Sampling } \\
\text { (CS) }\end{array}$ & $\begin{array}{l}\text { Women Entering } \\
\text { a Military } \\
\text { Substance } \\
\text { Use Disorder }\end{array}$ & Depression Checklist & - & 86 & NR & $15(17.4 \%)$ & 5 \\
\hline \multirow[t]{3}{*}{$\begin{array}{l}\text { Kline, A. et al. } \\
\text { (2009)(109) }\end{array}$} & \multirow[t]{3}{*}{ USA } & \multirow[t]{3}{*}{$\begin{array}{l}\text { Convenience } \\
\text { Sampling } \\
\text { (CS) }\end{array}$} & $\begin{array}{l}\text { Vietnam veterans } \\
\text { with Substance } \\
\text { Use Disorder }\end{array}$ & \multirow[t]{3}{*}{ SCID DSM-IV Diagnoses } & 55.20 & 82 & $\begin{array}{l}23 \\
(27.8 \%)\end{array}$ & $5(6.1 \%)$ & 8 \\
\hline & & & $\begin{array}{l}\text { Post-Vietnam } \\
\text { veterans with } \\
\text { Substance } \\
\text { Use Disorder }\end{array}$ & & 46.76 & 236 & $\begin{array}{l}63 \\
(26.8 \%)\end{array}$ & $16(6.8 \%)$ & \\
\hline & & & $\begin{array}{l}\text { Persian Gulf } \\
\text { veterans with } \\
\text { Substance } \\
\text { Use Disorder }\end{array}$ & & 34 & 55 & $9(15.4 \%)$ & $5(9.1 \%)$ & \\
\hline $\begin{array}{l}\text { Rehn, L. M. } \\
\text { et al. } \\
(2009)(110)\end{array}$ & Finland & $\begin{array}{l}\text { Convenience } \\
\text { Sampling } \\
\text { (CS) }\end{array}$ & $\begin{array}{l}\text { Male Finnish } \\
\text { military } \\
\text { conscripts }\end{array}$ & $\begin{array}{l}\text { The Beck Depression Inventory } \\
\text { (BDI) }\end{array}$ & 20 & 126 & NR & $9(7.1 \%)$ & 6 \\
\hline $\begin{array}{l}\text { Belik, S. L. et al. } \\
(2010)(199)\end{array}$ & Canada & $\begin{array}{l}\text { Convenience } \\
\text { Sampling } \\
\text { (CS) }\end{array}$ & $\begin{array}{l}\text { The Canadian } \\
\text { Forces }\end{array}$ & $\begin{array}{l}\text { Diagnostic and Statistical Manual } \\
\text { of Mental Disorders-IV } \\
\text { (DSM-IV) }\end{array}$ & - & 37,129 & $\begin{array}{l}236 \\
(0.8 \%)\end{array}$ & $\begin{array}{l}1613 \\
(4.34 \%)\end{array}$ & 8 \\
\hline $\begin{array}{l}\text { Guerra, V. S. } \\
\text { et al. } \\
(2011)(120)\end{array}$ & USA & $\begin{array}{l}\text { Convenience } \\
\text { Sampling } \\
\text { (CS) }\end{array}$ & $\begin{array}{l}\text { Veterans in } \\
\text { Operations } \\
\text { Enduring } \\
\text { Freedom and } \\
\text { Iraqi Freedom } \\
\text { (OEF/OIF) }\end{array}$ & $\begin{array}{l}\text { The Beck Depression Inventory } \\
\text { (BDI) } \\
\text { Beck Scale for Suicide Ideation } \\
\text { Scale for Suicide Ideation-Adapted }\end{array}$ & 38.3 & 393 & 34 (8.7\%) & $45(11.5 \%)$ & 8 \\
\hline \multirow{3}{*}{$\begin{array}{l}\text { Mansfield, A. J. } \\
\text { et al. } \\
(2011)(200)\end{array}$} & \multirow[t]{3}{*}{ USA } & \multirow{3}{*}{$\begin{array}{l}\text { Convenience } \\
\text { Sampling } \\
\text { (CS) }\end{array}$} & Military Personnel & \multirow{3}{*}{$\begin{array}{l}\text { The Center for } \\
\text { Epidemiological Studies } \\
\text { Depression (CES-D) scale, } \\
\text { The PRIME-MD } \\
\text { Patient Health }\end{array}$} & 28.1 & 3069 & \multirow[t]{3}{*}{ NR } & $215(7 \%)$ & \multirow[t]{3}{*}{6} \\
\hline & & & Military Personnel & & 31.8 & 1843 & & $98(5.3 \%)$ & \\
\hline & & & Military & & 25.8 & 1226 & & $110(9 \%)$ & \\
\hline
\end{tabular}


Table 2 The study characteristics of included studies about suicide attempted and thought (Continued)

\begin{tabular}{|c|c|c|c|c|c|c|c|c|c|}
\hline \multirow[t]{2}{*}{$\begin{array}{l}\text { Authors } \\
\text { (Years) }\end{array}$} & \multirow[t]{2}{*}{ Country } & \multirow{2}{*}{$\begin{array}{l}\text { Type of } \\
\text { Sampling } \\
\text { (Type of } \\
\text { Study) }\end{array}$} & \multirow[t]{2}{*}{$\begin{array}{l}\text { Study } \\
\text { Population }\end{array}$} & \multirow[t]{2}{*}{$\begin{array}{l}\text { Depression Assessment } \\
\text { Method }\end{array}$} & \multirow[t]{2}{*}{$\begin{array}{l}\text { Age } \\
\text { (Mean) }\end{array}$} & \multirow[t]{2}{*}{$\begin{array}{l}\text { Sample } \\
\text { size }\end{array}$} & \multicolumn{2}{|c|}{$\begin{array}{l}\text { Prevalence of } \\
\text { Suicide (\%) }\end{array}$} & \multirow[t]{2}{*}{$\begin{array}{l}\text { NOS } \\
\text { Score }\end{array}$} \\
\hline & & & & & & & Attempts & Thoughts & \\
\hline & & & $\begin{array}{l}\text { Personnel } \\
\text { (Marine) }\end{array}$ & $\begin{array}{l}\text { Questionnaire } \\
\text { (PHQ) }\end{array}$ & & & & & \\
\hline & & & & & & $\begin{array}{l}\text { Female } \\
(7255)\end{array}$ & & & \\
\hline $\begin{array}{l}\text { Maguen, S. } \\
\text { et al. } \\
\text { (2012)(201) }\end{array}$ & USA & $\begin{array}{l}\text { Convenience } \\
\text { Sampling } \\
\text { (CS) }\end{array}$ & Vietnam veterans & Checklist & 40 & 244 & $12(4.9 \%)$ & 40 (16.4\%) & 6 \\
\hline $\begin{array}{l}\text { Swinkels, C. M. } \\
\text { et al. } \\
\text { (2013)(130) }\end{array}$ & UK & $\begin{array}{l}\text { Convenience } \\
\text { Sampling } \\
\text { (CS) }\end{array}$ & $\begin{array}{l}\text { U.S. Afghanistan/ } \\
\text { Iraq Veterans }\end{array}$ & $\begin{array}{l}\text { Diagnostic and Statistical Manual } \\
\text { of Mental Disorders-IV } \\
\text { (DSM-IV) }\end{array}$ & 37.40 & 1640 & 132 (8\%) & NR & 6 \\
\hline $\begin{array}{l}\text { Bryan, C. J. } \\
\text { et al. } \\
(2013)(202)\end{array}$ & USA & $\begin{array}{l}\text { Convenience } \\
\text { Sampling } \\
\text { (CS) }\end{array}$ & $\begin{array}{l}\text { Deployed Military } \\
\text { Personnel }\end{array}$ & $\begin{array}{l}\text { The 4-item Suicidal Behaviors } \\
\text { Questionnaire- Revised (SBQ-R) }\end{array}$ & - & 161 & NR & 35 (21.7\%) & 5 \\
\hline $\begin{array}{l}\text { Bryan, C. J. } \\
\text { et al. } \\
(2013)(203)\end{array}$ & USA & $\begin{array}{l}\text { Convenience } \\
\text { Sampling } \\
\text { (CS) }\end{array}$ & $\begin{array}{l}\text { Deployed Military } \\
\text { Personnel }\end{array}$ & $\begin{array}{l}\text { The 4-item Suicidal Behaviors } \\
\text { Questionnaire- Revised (SBQ-R) }\end{array}$ & - & 158 & $3(1.5 \%)$ & $21(13.1 \%)$ & 5 \\
\hline $\begin{array}{l}\text { Bryan, C. J. } \\
\text { et al. } \\
(2013)(204)\end{array}$ & USA & $\begin{array}{l}\text { Convenience } \\
\text { Sampling } \\
\text { (CS) }\end{array}$ & $\begin{array}{l}\text { Air } \\
\text { Force Personnel }\end{array}$ & $\begin{array}{l}\text { Beck Scale for Suicidal Ideation- } \\
\text { Current } \\
\text { (BSSI-C) }\end{array}$ & 25.9 & 273 & NR & 53 (19.4\%) & 5 \\
\hline $\begin{array}{l}\text { Bryan, C. J. } \\
\text { et al. } \\
\text { (2013)(205) }\end{array}$ & USA & $\begin{array}{l}\text { Convenience } \\
\text { Sampling } \\
\text { (CS) }\end{array}$ & $\begin{array}{l}\text { Deployed Military } \\
\text { Personnel }\end{array}$ & $\begin{array}{l}\text { The Self-Injurious Thoughts and } \\
\text { Behaviors Interview (SITBI) }\end{array}$ & 34.2 & 69 & NR & $25(36.2 \%)$ & 5 \\
\hline $\begin{array}{l}\text { Blosnich, J. R. } \\
\text { et al. } \\
\text { (2014)(206) }\end{array}$ & USA & $\begin{array}{l}\text { Convenience } \\
\text { Sampling } \\
\text { (CS) }\end{array}$ & $\begin{array}{l}\text { Deployed Military } \\
\text { Personnel }\end{array}$ & Checklist & - & 4250 & NR & 154 (3.3\%) & 5 \\
\hline $\begin{array}{l}\text { Bryan, C. J. } \\
\text { et al. } \\
(2014)(207)\end{array}$ & USA & $\begin{array}{l}\text { Convenience } \\
\text { Sampling } \\
\text { (CS) }\end{array}$ & $\begin{array}{l}\text { Deployed Military } \\
\text { Personnel }\end{array}$ & $\begin{array}{l}\text { The Self-Injurious Thoughts and } \\
\text { Behaviors Interview (SITBI) }\end{array}$ & 36.7 & 374 & $29(7.8 \%)$ & $\begin{array}{l}136 \\
(36.4 \%)\end{array}$ & 6 \\
\hline $\begin{array}{l}\text { Mash, H. B. } \\
\text { et al. } \\
(2014)(208)\end{array}$ & USA & $\begin{array}{l}\text { Convenience } \\
\text { Sampling } \\
\text { (CS) }\end{array}$ & US Army & Checklist & - & 4999 & NR & $303(6 \%)$ & 6 \\
\hline $\begin{array}{l}\text { Don } \\
\text { Richardson, J. } \\
\text { et al. } \\
(2014)(135)\end{array}$ & Canada & $\begin{array}{l}\text { Convenience } \\
\text { Sampling } \\
\text { (CS) }\end{array}$ & $\begin{array}{l}\text { Canadian Forces } \\
\text { members and } \\
\text { veterans }\end{array}$ & $\begin{array}{l}\text { The PRIME-MD } \\
\text { Patient Health } \\
\text { Questionnaire } \\
\text { (PHQ) }\end{array}$ & - & 404 & $N R$ & 68 (16.8\%) & 7 \\
\hline $\begin{array}{l}\text { Ramsawh, H. J. } \\
\text { et al. } \\
(2014)(139)\end{array}$ & USA & $\begin{array}{l}\text { Convenience } \\
\text { Sampling } \\
\text { (CS) }\end{array}$ & $\begin{array}{l}\text { Active Duty } \\
\text { Military } \\
\text { Personnel }\end{array}$ & $\begin{array}{l}\text { 10-item Center for Epidemiologic } \\
\text { Studies Depression Scale }\end{array}$ & 35 & 5461 & $\begin{array}{l}346 \\
(6.33 \%)\end{array}$ & NR & 8 \\
\hline $\begin{array}{l}\text { Bryan, C. J. } \\
\text { et al. } \\
(2015)(209)\end{array}$ & USA & $\begin{array}{l}\text { Convenience } \\
\text { Sampling } \\
\text { (CS) }\end{array}$ & $\begin{array}{l}\text { Air Force } \\
\text { personnel }\end{array}$ & $\begin{array}{l}\text { The Suicidal Behaviors } \\
\text { Questionnaire Revised (SBQ-R) }\end{array}$ & - & 168 & $2(1.2 \%)$ & $29(17.3 \%)$ & 7 \\
\hline $\begin{array}{l}\text { Cleveland, S. D. } \\
\text { et al. } \\
(2015)(141)\end{array}$ & USA & $\begin{array}{l}\text { Convenience } \\
\text { Sampling } \\
\text { (CS) }\end{array}$ & $\begin{array}{l}\text { Veterans and } \\
\text { Civilian College } \\
\text { Students }\end{array}$ & $\begin{array}{l}\text { Diagnostic and Statistical Manual } \\
\text { of Mental Disorders-IV } \\
\text { (DSM-IV) }\end{array}$ & - & 26,969 & $\begin{array}{l}282 \\
(1.07 \%)\end{array}$ & $\begin{array}{l}1730 \\
(6.54 \%)\end{array}$ & 8 \\
\hline $\begin{array}{l}\text { Kim, N. Y. et al. } \\
\text { (2015) (145) }\end{array}$ & USA & $\begin{array}{l}\text { Convenience } \\
\text { Sampling } \\
\text { (CS) }\end{array}$ & Korean Soldiers & $\begin{array}{l}\text { Scale for suicide ideation (SSI), } \\
\text { The Beck Depression Inventory } \\
\text { (BDI) }\end{array}$ & 21.3 & 414 & $N R$ & 80 (19.3\%) & 6 \\
\hline $\begin{array}{l}\text { Ursano, R. J. } \\
\text { et al. } \\
(2015)(210)\end{array}$ & USA & $\begin{array}{l}\text { Convenience } \\
\text { Sampling } \\
\text { (CS) }\end{array}$ & Soldiers & $\begin{array}{l}\text { The Columbia Suicidal Severity } \\
\text { Rating Scale (C-SSRS) }\end{array}$ & 20 & 38,237 & $\begin{array}{l}536 \\
(1.9 \%)\end{array}$ & $\begin{array}{l}5353 \\
(14 \%)\end{array}$ & 8 \\
\hline $\begin{array}{l}\text { Vanderploeg, } \\
\text { R. D. et al. } \\
(2015) \text { (150) }\end{array}$ & USA & $\begin{array}{l}\text { Convenience } \\
\text { Sampling } \\
\text { (CS) }\end{array}$ & $\begin{array}{l}\text { Florida National } \\
\text { Guard } \\
\text { Members }\end{array}$ & $\begin{array}{l}\text { The PRIME-MD } \\
\text { Patient Health } \\
\text { Questionnaire } \\
\text { (PHQ) }\end{array}$ & - & 3098 & $N R$ & 130 (4.2\%) & 7 \\
\hline Forbes, D. & Australia & Convenience & Australian & Diagnostic and Statistical Manual & 46.5 & 2050 & $25(1.2 \%)$ & 275 & 8 \\
\hline
\end{tabular}


Table 2 The study characteristics of included studies about suicide attempted and thought (Continued)

\begin{tabular}{|c|c|c|c|c|c|c|c|c|c|}
\hline \multirow[t]{2}{*}{$\begin{array}{l}\text { Authors } \\
\text { (Years) }\end{array}$} & \multirow[t]{2}{*}{ Country } & \multirow{2}{*}{$\begin{array}{l}\text { Type of } \\
\text { Sampling } \\
\text { (Type of } \\
\text { Study) }\end{array}$} & \multirow[t]{2}{*}{$\begin{array}{l}\text { Study } \\
\text { Population }\end{array}$} & \multirow[t]{2}{*}{$\begin{array}{l}\text { Depression Assessment } \\
\text { Method }\end{array}$} & \multirow[t]{2}{*}{$\begin{array}{l}\text { Age } \\
\text { (Mean) }\end{array}$} & \multirow[t]{2}{*}{$\begin{array}{l}\text { Sample } \\
\text { size }\end{array}$} & \multicolumn{2}{|c|}{$\begin{array}{l}\text { Prevalence of } \\
\text { Suicide (\%) }\end{array}$} & \multirow[t]{2}{*}{$\begin{array}{l}\text { NOS } \\
\text { Score }\end{array}$} \\
\hline & & & & & & & Attempts & Thoughts & \\
\hline $\begin{array}{l}\text { et al. } \\
(2016)(152)\end{array}$ & & $\begin{array}{l}\text { Sampling } \\
\text { (CS) }\end{array}$ & peacekeepers & $\begin{array}{l}\text { of Mental Disorders-IV } \\
\text { (DSM-IV) }\end{array}$ & & & & $(13.4 \%)$ & \\
\hline \multirow{2}{*}{$\begin{array}{l}\text { Herberman } \\
\text { Mash, H. B. } \\
\text { et al. } \\
(2016)(155)\end{array}$} & \multirow[t]{2}{*}{ USA } & \multirow{2}{*}{$\begin{array}{l}\text { Convenience } \\
\text { Sampling } \\
\text { (CS) }\end{array}$} & U.S. Army soldiers & \multirow{2}{*}{$\begin{array}{l}\text { The 10-item } \\
\text { Center for Epidemiologic Studies } \\
\text { Depression Scale }\end{array}$} & \multirow[t]{2}{*}{-} & 3813 & $230(6 \%)$ & \multirow[t]{2}{*}{ NR } & \multirow[t]{2}{*}{8} \\
\hline & & & $\begin{array}{l}\text { U.S. Army soldiers } \\
\text { with alcohol use }\end{array}$ & & & 1210 & $\begin{array}{l}100 \\
(8.3 \%)\end{array}$ & & \\
\hline \multirow{3}{*}{$\begin{array}{l}\text { Monteith, L. L. } \\
\text { et al. } \\
(2016)(156)\end{array}$} & \multirow[t]{3}{*}{ USA } & \multirow{3}{*}{$\begin{array}{l}\text { Convenience } \\
\text { Sampling } \\
\text { (CS) }\end{array}$} & \multirow[t]{3}{*}{ Veterans } & \multirow{3}{*}{$\begin{array}{l}\text { Beck Scale for Suicide Ideation } \\
\text { (BSS), } \\
\text { Multidimensional Suicide } \\
\text { Inventory-28 (MSI) Negative Affect } \\
\text { scale }\end{array}$} & 49.6 & $\begin{array}{l}\text { Total } \\
(354)\end{array}$ & $\begin{array}{l}92 \\
(26.8 \%)\end{array}$ & \multirow[t]{3}{*}{ NR } & \multirow[t]{3}{*}{8} \\
\hline & & & & & & $\begin{array}{l}\text { Male } \\
(310)\end{array}$ & $\begin{array}{l}82 \\
(26.5 \%)\end{array}$ & & \\
\hline & & & & & & $\begin{array}{l}\text { Female } \\
(44)\end{array}$ & $\begin{array}{l}13 \\
(29.5 \%)\end{array}$ & & \\
\hline \multirow{2}{*}{$\begin{array}{l}\text { Cohen, G. H. } \\
\text { et al. } \\
(2017)(160)\end{array}$} & \multirow[t]{2}{*}{ USA } & \multirow{2}{*}{$\begin{array}{l}\text { Convenience } \\
\text { Sampling } \\
\text { (CS) }\end{array}$} & $\begin{array}{l}\text { Army National } \\
\text { Guard Soldiers }\end{array}$ & \multirow{2}{*}{$\begin{array}{l}\text { The PRIME-MD } \\
\text { Patient Health } \\
\text { Questionnaire } \\
\text { (PHQ), } \\
\text { The PHQ-9 Item }\end{array}$} & \multirow[t]{2}{*}{-} & 1582 & \multirow[t]{2}{*}{$N R$} & $42(2.6 \%)$ & \multirow[t]{2}{*}{8} \\
\hline & & & $\begin{array}{l}\text { Army National } \\
\text { with Alcohol Use }\end{array}$ & & & 93 & & $8(8.6 \%)$ & \\
\hline \multirow{3}{*}{$\begin{array}{l}\text { Gradus, J. L. } \\
\text { et al. } \\
\text { (2017)(161) }\end{array}$} & \multirow[t]{3}{*}{ USA } & \multirow{3}{*}{$\begin{array}{l}\text { Random } \\
\text { Sampling } \\
\text { (CS) }\end{array}$} & $\begin{array}{l}\text { Veterans of the } \\
\text { Iraq and }\end{array}$ & $\begin{array}{l}\text { 20- } \\
\text { item, self-report Center for Epi- }\end{array}$ & 34 & $\begin{array}{l}\text { Total } \\
(2244)\end{array}$ & NR & $\begin{array}{l}370 \\
(16.5 \%)\end{array}$ & 7 \\
\hline & & & & $\begin{array}{l}\text { Scale (CES-D), } \\
\text { The 4-item Suicidal Behaviors }\end{array}$ & & $\begin{array}{l}\text { Male } \\
(1062)\end{array}$ & & $\begin{array}{l}179 \\
(16.9 \%)\end{array}$ & \\
\hline & & & & $\begin{array}{l}\text { Questionnaire-Short Form (SBQ- } \\
\text { SF) }\end{array}$ & & $\begin{array}{l}\text { Female } \\
\text { (1099) }\end{array}$ & & $\begin{array}{l}191 \\
(17.4 \%)\end{array}$ & \\
\hline $\begin{array}{l}\text { Weeks, M. } \\
\text { et al. } \\
(2017)(163)\end{array}$ & Canada & $\begin{array}{l}\text { Convenience } \\
\text { Sampling } \\
\text { (CS) }\end{array}$ & $\begin{array}{l}\text { Canadian Military } \\
\text { and Civilian } \\
\text { Populations }\end{array}$ & $\begin{array}{l}\text { Diagnostic and Statistical Manual } \\
\text { of Mental Disorders-IV } \\
\text { (DSM-IV) }\end{array}$ & 35 & 6696 & NR & 289 (4. \%) & 8 \\
\hline $\begin{array}{l}\text { Bartlett, B. A. } \\
\text { et al. } \\
(2018)(164)\end{array}$ & USA & $\begin{array}{l}\text { Convenience } \\
\text { Sampling } \\
\text { (CS) }\end{array}$ & Military veterans & $\begin{array}{l}20- \\
\text { item, self-report Center for Epi- } \\
\text { demiological Studies Depression } \\
\text { Scale (CES-D) }\end{array}$ & 38.40 & 910 & $62(7.5 \%)$ & NR & 6 \\
\hline $\begin{array}{l}\text { Boulos, D. et al. } \\
\text { (2018)(166) }\end{array}$ & Canada & $\begin{array}{l}\text { Random } \\
\text { Sampling }\end{array}$ & $\begin{array}{l}\text { Regular Force } \\
\text { personnel }\end{array}$ & $\begin{array}{l}\text { Diagnostic and Statistical Manual } \\
\text { of Mental Disorders-IV }\end{array}$ & - & 3385 & NR & $156(4.6 \%)$ & 7 \\
\hline & & & $\begin{array}{l}\text { Reserve Force } \\
\text { personnel }\end{array}$ & & & 1469 & & $82(5.6 \%)$ & \\
\hline $\begin{array}{l}\text { Dillon, K. H. } \\
\text { et al. } \\
(2018)(167)\end{array}$ & USA & $\begin{array}{l}\text { Convenience } \\
\text { Sampling } \\
\text { (CS) }\end{array}$ & $\begin{array}{l}\text { Iraq/Afghanistan- } \\
\text { era veterans }\end{array}$ & $\begin{array}{l}\text { The Beck Scale for Suicide } \\
\text { Ideation (BSS), The Structured } \\
\text { Clinical Interview for DSM-IV-TR } \\
\text { (SCID) }\end{array}$ & - & 3238 & 291 (9\%) & NR & 7 \\
\hline $\begin{array}{l}\text { Elbogen, E. B. } \\
\text { et al. } \\
(2018)(169)\end{array}$ & USA & $\begin{array}{l}\text { Convenience } \\
\text { Sampling } \\
\text { (CS) }\end{array}$ & $\begin{array}{l}\text { Iraq/Afghanistan- } \\
\text { era veterans }\end{array}$ & $\begin{array}{l}\text { Diagnostic and Statistical Manual } \\
\text { of Mental Disorders-IV } \\
\text { (DSM-IV) }\end{array}$ & 34.9 & 1172 & $87(7.5 \%)$ & $N R$ & 6 \\
\hline $\begin{array}{l}\text { Hourani, L. L. } \\
\text { et al. } \\
(2018)(170)\end{array}$ & USA & $\begin{array}{l}\text { Convenience } \\
\text { Sampling } \\
\text { (CS) }\end{array}$ & $\begin{array}{l}\text { Active duty } \\
\text { military personnel }\end{array}$ & $\begin{array}{l}\text { The PRIME-MD } \\
\text { Patient Health } \\
\text { Questionnaire } \\
\text { (PHQ), Checklist }\end{array}$ & - & 947 & $16(2.1 \%)$ & $71(9.2 \%)$ & 7 \\
\hline $\begin{array}{l}\text { Kachadourian, } \\
\text { L. K. et al. } \\
(2018)(211)\end{array}$ & USA & $\begin{array}{l}\text { Convenience } \\
\text { Sampling } \\
\text { (CS) }\end{array}$ & Veterans & $\begin{array}{l}\text { The Columbia-Suicide Severity } \\
\text { Rating Scale (C-SSRS) }\end{array}$ & 43.9 & 93 & $\begin{array}{l}19 \\
(21.6 \%)\end{array}$ & $N R$ & 6 \\
\hline $\begin{array}{l}\text { Kerr, K. et al. } \\
(2018)(212)\end{array}$ & Australia & $\begin{array}{l}\text { Convenience } \\
\text { Sampling } \\
\text { (CS) }\end{array}$ & $\begin{array}{l}\text { Australian } \\
\text { veterans }\end{array}$ & Checklist & 54.6 & 229 & $\begin{array}{l}54 \\
(23.6 \%)\end{array}$ & $N R$ & 6 \\
\hline $\begin{array}{l}\text { Waitzkin, } \mathrm{H} \text {. } \\
\text { et al. } \\
\text { (2018)(175) }\end{array}$ & USA & $\begin{array}{l}\text { Convenience } \\
\text { Sampling } \\
\text { (CS) }\end{array}$ & Military Personnel & $\begin{array}{l}\text { The PRIME-MD } \\
\text { Patient Health } \\
\text { Questionnaire } \\
\text { (PHQ) }\end{array}$ & - & 198 & NR & $92(48 \%)$ & 7 \\
\hline
\end{tabular}


Table 2 The study characteristics of included studies about suicide attempted and thought (Continued)

\begin{tabular}{|c|c|c|c|c|c|c|c|c|c|}
\hline \multirow[t]{2}{*}{$\begin{array}{l}\text { Authors } \\
\text { (Years) }\end{array}$} & \multirow[t]{2}{*}{ Country } & \multirow{2}{*}{$\begin{array}{l}\text { Type of } \\
\text { Sampling } \\
\text { (Type of } \\
\text { Study) }\end{array}$} & \multirow[t]{2}{*}{$\begin{array}{l}\text { Study } \\
\text { Population }\end{array}$} & \multirow[t]{2}{*}{$\begin{array}{l}\text { Depression Assessment } \\
\text { Method }\end{array}$} & \multirow[t]{2}{*}{$\begin{array}{l}\text { Age } \\
\text { (Mean) }\end{array}$} & \multirow[t]{2}{*}{$\begin{array}{l}\text { Sample } \\
\text { size }\end{array}$} & \multicolumn{2}{|c|}{$\begin{array}{l}\text { Prevalence of } \\
\text { Suicide (\%) }\end{array}$} & \multirow[t]{2}{*}{$\begin{array}{l}\text { NOS } \\
\text { Score }\end{array}$} \\
\hline & & & & & & & Attempts & Thoughts & \\
\hline $\begin{array}{l}\text { Byrne, S. P. } \\
\text { et al. } \\
(2019)(176)\end{array}$ & USA & $\begin{array}{l}\text { Convenience } \\
\text { Sampling } \\
\text { (CS) }\end{array}$ & $\begin{array}{l}\text { U.S. military } \\
\text { veterans }\end{array}$ & $\begin{array}{l}\text { The PRIME-MD } \\
\text { Patient Health } \\
\text { Questionnaire } \\
\text { (PHQ) }\end{array}$ & 53.4 & 158 & $\begin{array}{l}40 \\
(24.4 \%)\end{array}$ & 39 (30.2\%) & 7 \\
\hline $\begin{array}{l}\text { Nichter, B. } \\
\text { et al. } \\
(2019)(180)\end{array}$ & USA & $\begin{array}{l}\text { Random } \\
\text { Sampling } \\
\text { (CS) }\end{array}$ & $\begin{array}{l}\text { U.S. } \\
\text { veteran } \\
\text { population }\end{array}$ & $\begin{array}{l}\text { The Patient Health } \\
\text { Questionnaire-4 (PHQ-4), The } \\
\text { Patient Health } \\
\text { Questionnaire-9 (PHQ-9) }\end{array}$ & 60.3 & 2732 & $\begin{array}{l}134 \\
(4.9 \%)\end{array}$ & $248(9 \%)$ & 9 \\
\hline $\begin{array}{l}\text { Start, A. R. } \\
\text { et al. } \\
\text { (2019)(181) }\end{array}$ & USA & $\begin{array}{l}\text { Convenience } \\
\text { Sampling } \\
\text { (CS) }\end{array}$ & Military Personnel & $\begin{array}{l}\text { The Patient Health } \\
\text { Questionnaire-9 (PHQ-9) }\end{array}$ & - & 944 & $N R$ & $31(3.3 \%)$ & 7 \\
\hline $\begin{array}{l}\text { Blosnich, J. R. } \\
\text { et al. } \\
(2020)(182)\end{array}$ & USA & $\begin{array}{l}\text { Random } \\
\text { Sampling } \\
\text { (CS) }\end{array}$ & Military Veterans & $\begin{array}{l}\text { Diagnostic and Statistical Manual } \\
\text { of Mental Disorders-IV } \\
\text { (DSM-IV) }\end{array}$ & - & 293,872 & $\begin{array}{l}1035 \\
(0.3 \%)\end{array}$ & $2999(1 \%)$ & 9 \\
\hline $\begin{array}{l}\text { Cramer, R. J. } \\
\text { et al. } \\
\text { (2020)(213) }\end{array}$ & USA & $\begin{array}{l}\text { Random } \\
\text { Sampling } \\
\text { (CS) }\end{array}$ & Military Personnel & $\begin{array}{l}\text { The Suicide Behaviors } \\
\text { Questionnaire-Revised (SBQ-R) }\end{array}$ & - & 200 & $96(48 \%)$ & NR & 6 \\
\hline $\begin{array}{l}\text { Groll, D. L. } \\
\text { et al. } \\
(2020)(185)\end{array}$ & Canada & $\begin{array}{l}\text { Convenience } \\
\text { Sampling } \\
\text { (CS) }\end{array}$ & $\begin{array}{l}\text { Canadian military } \\
\text { persons }\end{array}$ & $\begin{array}{l}\text { The Patient Health } \\
\text { Questionnaire-9 (PHQ-9) }\end{array}$ & - & 477 & $19(4 \%)$ & $76(16 \%)$ & 8 \\
\hline $\begin{array}{l}\text { Shim, E. J. et al. } \\
(2020)(187)\end{array}$ & Korea & $\begin{array}{l}\text { Random } \\
\text { Sampling } \\
\text { (CS) }\end{array}$ & $\begin{array}{l}\text { Korean military } \\
\text { population }\end{array}$ & $\begin{array}{l}\text { The Mini } \\
\text { International Neuropsychiatric } \\
\text { Interview Plus (MINI-Plus), The } \\
\text { Patient Health } \\
\text { Questionnaire-9 (PHQ-9) }\end{array}$ & 50.6 & 1937 & 87 (4.5\%) & NR & 8 \\
\hline $\begin{array}{l}\text { Smigelsky, M. } \\
\text { A. et al. } \\
(2020)(188)\end{array}$ & USA & $\begin{array}{l}\text { Convenience } \\
\text { Sampling } \\
\text { (CS) }\end{array}$ & $\begin{array}{l}\text { U.S. military } \\
\text { population }\end{array}$ & $\begin{array}{l}\text { Diagnostic and Statistical Manual } \\
\text { of Mental Disorders-IV } \\
\text { (DSM-IV) }\end{array}$ & 37.6 & 1002 & $41(4 \%)$ & NR & 6 \\
\hline $\begin{array}{l}\text { Stefanovics, E. } \\
\text { A. et al. } \\
(2020)(190)\end{array}$ & USA & $\begin{array}{l}\text { Convenience } \\
\text { Sampling } \\
\text { (CS) }\end{array}$ & $\begin{array}{l}\text { U.S. Military } \\
\text { Veterans }\end{array}$ & $\begin{array}{l}\text { The Mini International } \\
\text { Neuropsychiatric Interview (MINI), } \\
\text { The Patient Health } \\
\text { Questionnaire-9 (PHQ-9) }\end{array}$ & 55 & 1308 & $118(9 \%)$ & $165(12.6 \%)$ & 5 \\
\hline $\begin{array}{l}\text { Wang, J. et al. } \\
(2020)(192)\end{array}$ & USA & $\begin{array}{l}\text { Convenience } \\
\text { Sampling } \\
\text { (CS) }\end{array}$ & $\begin{array}{l}\text { U.S. } \\
\text { Reserve and } \\
\text { National Guard } \\
\text { Personnel }\end{array}$ & $\begin{array}{l}\text { The Patient Health } \\
\text { Questionnaire-9 (PHQ-9) }\end{array}$ & 34.4 & 3503 & $N R$ & $101(2.9 \%)$ & 6 \\
\hline $\begin{array}{l}\text { Anestis, M. D. } \\
\text { et al. } \\
(2020)(214)\end{array}$ & USA & $\begin{array}{l}\text { Convenience } \\
\text { Sampling } \\
\text { (CS) }\end{array}$ & $\begin{array}{l}\text { U.S. Military } \\
\text { Veterans }\end{array}$ & $\begin{array}{l}\text { The Suicide Behaviors } \\
\text { Questionnaire-Revised (SBQ-R) }\end{array}$ & 27.0 & 953 & $N R$ & $\begin{array}{l}105 \\
(15.2 \%)\end{array}$ & 5 \\
\hline $\begin{array}{l}\text { Monteith, L. L. } \\
\text { et al. } \\
(2020)(215)\end{array}$ & USA & $\begin{array}{l}\text { Convenience } \\
\text { Sampling } \\
\text { (CS) }\end{array}$ & Female veterans & Checklist & 55.6 & 439 & 158(36\%) & $113(25.7 \%)$ & 5 \\
\hline $\begin{array}{l}\text { Ursano, R. J. } \\
\text { et al. } \\
(2020)(193)\end{array}$ & USA & $\begin{array}{l}\text { Convenience } \\
\text { Sampling } \\
\text { (CS) }\end{array}$ & $\begin{array}{l}\text { US Army Soldiers } \\
\text { During } \\
\text { Deployment } \\
\text { in Afghanistan }\end{array}$ & $\begin{array}{l}\text { Diagnostic and Statistical Manual } \\
\text { of Mental Disorders-IV } \\
\text { (DSM-IV) }\end{array}$ & - & 3957 & $N R$ & $85(2.1 \%)$ & 7 \\
\hline $\begin{array}{l}\text { Yeom, C. W. } \\
\text { et al. } \\
(2020)(194)\end{array}$ & Korea & $\begin{array}{l}\text { Convenience } \\
\text { Sampling } \\
\text { (CS) }\end{array}$ & $\begin{array}{l}\text { Korean military } \\
\text { personal }\end{array}$ & $\begin{array}{l}\text { The Mini } \\
\text { International Neuropsychiatric } \\
\text { Interview Plus } \\
\text { (MINI-Plus Suicidality module), The } \\
\text { Patient Health } \\
\text { Questionnaire-9 (PHQ-9) }\end{array}$ & 21.4 & 480 & $22(4.5 \%)$ & $N R$ & 6 \\
\hline
\end{tabular}


Table 3 The pooled estimate of prevalence of depression in active duty and veteran military

\begin{tabular}{|c|c|c|c|c|c|c|c|}
\hline \multirow[t]{2}{*}{ Categories } & \multirow{2}{*}{$\begin{array}{l}\text { No. of } \\
\text { Studies } \\
\text { (Sample } \\
\text { Size) }\end{array}$} & \multirow{2}{*}{$\begin{array}{l}\text { Pooled } \\
\text { Prevalence } \\
\text { (\% 95 Cl) }\end{array}$} & \multicolumn{3}{|c|}{$\begin{array}{l}\text { Between studies heterogeneity } \\
\text { assessment (\%) }\end{array}$} & \multicolumn{2}{|c|}{$\begin{array}{l}\text { Between subgroups } \\
\text { heterogeneity assessment (\%, }\end{array}$} \\
\hline & & & $P^{2}$ & $P_{\text {Heterogenity }}$ & $z$ & $\bar{Q}$ & $P_{\text {Heterogenity }}$ \\
\hline \multicolumn{8}{|c|}{ The prevalence of depression in active duty military } \\
\hline Total & $100(1278837)$ & $23 \%(20-26 \%)$ & $87.91 \%$ & 0.018 & 27.74 & - & - \\
\hline \multicolumn{8}{|l|}{ Sampling Method } \\
\hline Convinces Sampling & 67 (939796) & $21 \%(18-25 \%)$ & $66.90 \%$ & 0.030 & 20.25 & 9.33 & 0.001 \\
\hline Random Sampling & $33(339041)$ & $26 \%(19-32 \%)$ & $54.80 \%$ & 0.050 & 13.11 & & \\
\hline \multicolumn{8}{|l|}{ Type of Forces } \\
\hline Air Forces & $5(4562)$ & $20 \%(9-33 \%)$ & $83.93 \%$ & 0.040 & 5.59 & 8.98 & 0.001 \\
\hline Armed Forces & $36(995073)$ & $22 \%(20-23 \%)$ & $89.45 \%$ & 0.034 & 20.05 & & \\
\hline Marine Forces & $6(775778)$ & $31 \%(16-48 \%)$ & $90.86 \%$ & 0.0001 & 6.22 & & \\
\hline Military Forces & $53(201624)$ & $22 \%(16-28 \%)$ & $79.89 \%$ & 0.005 & 12.41 & & \\
\hline Population Healthy Forces & $90(1152451)$ & $22 \%(20-25 \%)$ & $99.87 \%$ & 0.0001 & 18.28 & 10.03 & 0.001 \\
\hline Forces with HIV/AIDS & $3(113620)$ & $15 \%(3-36 \%)$ & - & - & 3.16 & & \\
\hline Forces with Alcohol Use & $5(8303)$ & $29 \%(13-47 \%)$ & $99.96 \%$ & 0.0001 & 5.70 & & \\
\hline Forces with Substance Use & $2(4463)$ & $37 \%(36-39 \%)$ & - & - & 18.04 & & \\
\hline \multicolumn{8}{|l|}{ Gender } \\
\hline Total & 71 (1163273) & $22 \%(20-25 \%)$ & $90.88 \%$ & 0.0001 & 20.19 & 10.01 & 0.001 \\
\hline Male & $20(110847)$ & $23 \%(12-37 \%)$ & $91.83 \%$ & 0.0001 & 6.45 & & \\
\hline Female & $9(4717)$ & $25 \%(13-40 \%)$ & $89.99 \%$ & 0.012 & 6.15 & & \\
\hline \multicolumn{8}{|l|}{ Tools } \\
\hline BDI Scale & 9 (38888) & $25 \%(15-36 \%)$ & $65.75 \%$ & 0.054 & 5.88 & 5.09 & 0.001 \\
\hline CES-D Scale & $7(15365)$ & $13 \%(8-19 \%)$ & $50.20 \%$ & 0.130 & 4.05 & & \\
\hline Interviews & $13(16980)$ & $25 \%(17-35 \%)$ & $67.38 \%$ & 0.060 & 10.22 & & \\
\hline DSM-IV Scale & $36(202430)$ & $15 \%(11-19 \%)$ & $60.07 \%$ & 0.078 & 12.41 & & \\
\hline BSI Scale & $1(236)$ & $56 \%(49-60 \%)$ & - & - & - & & \\
\hline HAMD Scale & $1(197)$ & $47 \%(40-54 \%)$ & - & - & - & & \\
\hline HADS Scale & 1 (6943) & $10 \%(9-11 \%)$ & - & - & - & & \\
\hline PHQ Scale & 24 (692087) & $15 \%(13-17 \%)$ & $78.62 \%$ & 0.059 & 9.32 & & \\
\hline SDS Scale & $5(304767)$ & $20 \%(14-26 \%)$ & $72.99 \%$ & 0.059 & 9.03 & & \\
\hline \multicolumn{8}{|l|}{ Country } \\
\hline Canada & $10(318747)$ & $21 \%(16-26 \%)$ & $49.46 \%$ & 0.760 & 4.99 & & \\
\hline Korea & $2(430)$ & $20 \%(16-24 \%)$ & $0.0 \%$ & 0.782 & 1.49 & & \\
\hline Thailand & $2(2272)$ & $39 \%(37-41 \%)$ & $0.0 \%$ & 0.800 & 0.98 & 17.74 & 0.001 \\
\hline United Kingdom & $6(2034)$ & $32 \%(10-59 \%)$ & $54.32 \%$ & 0.763 & 4.05 & & \\
\hline USA & 66 (929016) & $21 \%(17-25 \%)$ & $78.96 \%$ & 0.050 & 13.54 & & \\
\hline Greece & $3(6845)$ & $20 \%(1-52 \%)$ & $0.0 \%$ & 0.980 & 2.43 & & \\
\hline \multicolumn{8}{|c|}{ The prevalence of depression in veteran military } \\
\hline Total & 80 (887982) & $20 \%(18-22 \%)$ & $79.80 \%$ & 0.032 & 31.46 & - & - \\
\hline \multicolumn{8}{|l|}{ Sampling Method } \\
\hline Convinces Sampling & 55 (565979) & $19 \%(16-21 \%)$ & $69.78 \%$ & 0.049 & 17.25 & 2.12 & 0.150 \\
\hline Random Sampling & 25 (322003) & $22 \%(18-27 \%)$ & $58.26 \%$ & 0.054 & 10.02 & & \\
\hline
\end{tabular}

Type of Forces

Air Forces

NR 
Table 3 The pooled estimate of prevalence of depression in active duty and veteran military (Continued)

\begin{tabular}{|c|c|c|c|c|c|c|c|}
\hline \multirow[t]{2}{*}{ Categories } & \multirow{2}{*}{$\begin{array}{l}\text { No. of } \\
\text { Studies } \\
\text { (Sample } \\
\text { Size) }\end{array}$} & \multirow{2}{*}{$\begin{array}{l}\text { Pooled } \\
\text { Prevalence } \\
\text { (\% } 95 \mathrm{Cl})\end{array}$} & \multicolumn{3}{|c|}{$\begin{array}{l}\text { Between studies heterogeneity } \\
\text { assessment (\%) }\end{array}$} & \multicolumn{2}{|c|}{$\begin{array}{l}\text { Between subgroups } \\
\text { heterogeneity assessment (\%) }\end{array}$} \\
\hline & & & $P^{2}$ & $P_{\text {Heterogenity }}$ & $Z$ & $\bar{Q}$ & $P_{\text {Heterogenity }}$ \\
\hline Armed Forces & $68(583048)$ & $19 \%(17-22 \%)$ & $76.97 \%$ & 0.054 & 16.88 & 1.27 & 0.260 \\
\hline Marine Forces & $N R$ & - & - & - & - & & \\
\hline Military Forces & $12(304934)$ & $24 \%(16-33 \%)$ & $64.66 \%$ & 0.034 & 9.83 & & \\
\hline \multicolumn{8}{|l|}{ Population } \\
\hline Healthy Forces & $64(856091)$ & $19 \%(17-22 \%)$ & $99.09 \%$ & 0.0001 & 18.28 & 28.40 & 0.001 \\
\hline Forces with HIV/AIDS & $2(1257)$ & $16 \%(14-18 \%)$ & $91.33 \%$ & 0.0001 & 22.32 & & \\
\hline Forces with Alcohol Use & $4(1780)$ & $29 \%(21-37 \%)$ & $98.44 \%$ & 0.0001 & 11.92 & & \\
\hline Forces with Substance Use & 4 (4397) & $10 \%(6-14 \%)$ & $74.50 \%$ & 0.0001 & 8.68 & & \\
\hline Forces with HCV & $6(24457)$ & $29 \%(17-43 \%)$ & 88.93 & 0.001 & 7.36 & & \\
\hline \multicolumn{8}{|l|}{ Gender } \\
\hline Total & $55(237654)$ & $20 \%(17-23 \%)$ & $90.88 \%$ & 0.0001 & 22.36 & 0.12 & 0.873 \\
\hline Male & $15(343584)$ & $21 \%(13-31 \%)$ & $91.91 \%$ & 0.0001 & 7.75 & & \\
\hline Female & $10(306744)$ & $20 \%(14-26 \%)$ & $88.49 \%$ & 0.0001 & 11.40 & & \\
\hline \multicolumn{8}{|l|}{ Tools } \\
\hline BDI Scale & $7(415692)$ & $14 \%$ (9-21\%) & $55.15 \%$ & 0.060 & 7.97 & & \\
\hline CES-D Scale & $11(318802)$ & $18 \%(13-25 \%)$ & $40.45 \%$ & 0.761 & 10.80 & & \\
\hline Interviews & $13(50675)$ & $20 \%(11-31 \%)$ & $60.22 \%$ & 0.181 & 6.74 & & \\
\hline DSM-IV Scale & $11(64263)$ & $15 \%(9-22 \%)$ & $78.99 \%$ & 0.028 & 7.54 & & \\
\hline PHQ Scale & 29 (28445) & $21 \%(17-25 \%)$ & $78.48 \%$ & 0.049 & 17.52 & & \\
\hline SDS Scale & $2(1300)$ & $47 \%(44-50 \%)$ & $52.04 \%$ & 0.059 & 9.14 & & \\
\hline GDS Scale & 1 (1032) & $37 \%$ (34-40\%) & & - & 20.91 & & \\
\hline MHI Scale & 4 (3649) & $35 \%(15-59 \%)$ & $-50.74 \%$ & 0.601 & 4.91 & & \\
\hline QIDS Scale & $1(1002)$ & $21 \%(18-24 \%)$ & - & - & 19.13 & & \\
\hline HDRS Scale & $1(3122)$ & $7 \%(6-8 \%)$ & - & - & 18.28 & 22.16 & 0.001 \\
\hline \multicolumn{8}{|l|}{ Country } \\
\hline Canada & $2(2365)$ & $13 \%(10-15 \%)$ & $0.0 \%$ & 0.880 & 3.85 & & \\
\hline Croatia & $2(118669)$ & $4 \%(6-8 \%)$ & $0.0 \%$ & 0.893 & 4.91 & 31.46 & 0.001 \\
\hline USA & 70 (733009) & $20 \%(18-22 \%)$ & $67.84 \%$ & 0.049 & 29.94 & & \\
\hline
\end{tabular}

Beck Depression Inventory (BDI), Center for Epidemiological Studies Depression (CES-D), Clinical Symptoms (Interviews), Diagnostic and Statistical Manual of Mental Disorders-IV (DSM-IV), The Brief Symptom Inventory (BSI), The Hamilton Depression Rating Scale (HAMD), The Hospital Anxiety and Depression Scale (HADS), The Patient Health Questionnaire (PHQ), The Zung Self-Report Depression Scale (SDS), Geriatric Depression Scale (GDS), Mental Health Inventory (MHI), Quick Inventory of Depressive Symptomatology (QIDS), Hamilton Depression Rating Scale (HDRS)-24 item

\section{Publication bias, and meta-regression in studies related to the active military}

The results of the publication bias were shown in Fig. 2 for studies related to the active military. The results of the Eggers test showed that diffusion bias did not occur in calculating the prevalence of depression in the active military (B: 0.96, SE: 0.69, P: 0.167) (Fig. 2). In metaregression analysis, the effect of military personnel age on prevalence was studied and analyzed. The results presented that age had a significant effect on the prevalence of depression in the active military and for every 1 year of age, depression increased by $0.04 \%$. The results of heterogeneity evaluation demonstrated that 5 studies were the cause of heterogeneity in the meta-analysis of the depression prevalence in active military (Fig. 2).

\section{Prevalence of depression in veterans}

Regarding the prevalence of depression in veterans, 80 cross-sectional articles with a sample size of 887,982 people were reviewed, of whom 822,967 people were depressed. After combining the results of these studies, the overall pooled prevalence of depression in veterans was $20 \%$ (\% 95 CI; 18-22\%). The percentage of heterogeneity was $99.80 \%$ which was statistically significant (Table 3 ).

The results of the subgroup analysis showed that 55 studies had used the convinces sampling method and 25 


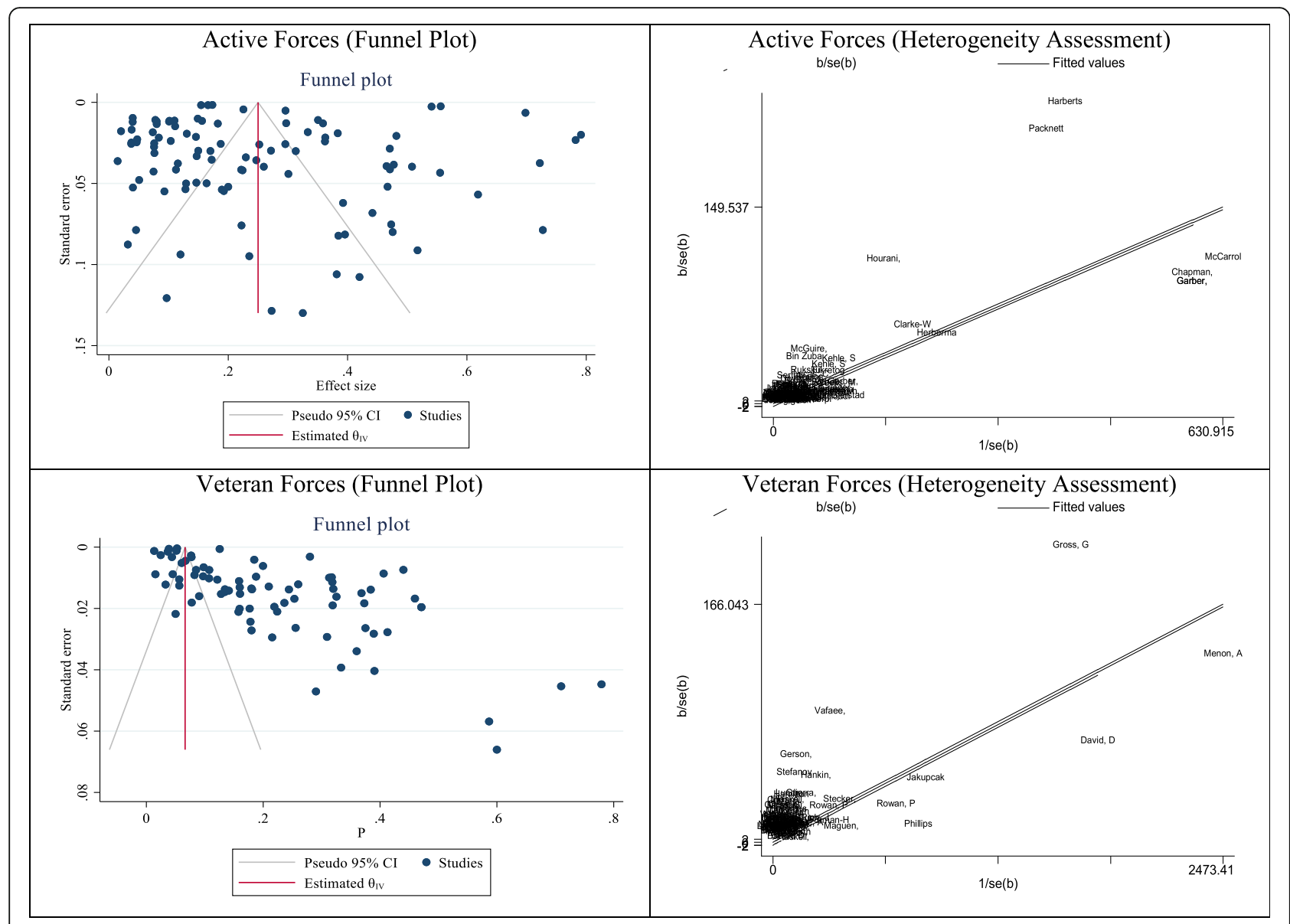

Fig. 2 Results of Publication bias and Heterogeneity in pooled prevalence of depression in active duty and veteran military

studies had used the random sampling method to determine the prevalence of depression in veterans. The sample size in the studies that had used the convinces sampling method was equal to 565,979 people. After combining their results, the pooled prevalence of depression was equal to $19 \%$ (\% $95 \mathrm{CI} ; 16-21 \%$ ). Also, the sample size in studies that had used the random sampling method was equal to 32,2003 people. After combining their results, the pooled prevalence of depression in veterans was equal to $22 \%$ (\% $95 \mathrm{CI} ; 18-27 \%$ ) (Table 3).

Regarding the military community of different divisions, the analysis showed that in the case of veterans, 68 studies had been conducted in the veterans' community of the Army, and 12 studies had been conducted in the entire military (without separating the different divisions). There was no study in the Air Force or Navy. The sample size in military veterans was 583,048 people and after combining these results, the pooled prevalence of depression was 19\% (\% 95 CI; 17-22\%) (Table 3).

The results of meta-analysis based on questionnaires and various measurement tools showed that heterogeneity of pooled prevalence was significantly reduced. In this section, 7 cross-sectional studies included in the meta-analysis using the beck depression inventory (BDI) questionnaire, 11 studies using the center for epidemiological studies depression (CES-D), 13 studies based on clinical criteria and interviews, 11 studies based on diagnostic and statistical manual of mental disorders-IV (DSM-IV), 29 studies based on the patient health questionnaire (PHQ), 2 studies based on the Zung self-report depression scale (SDS), 4 studies based on mental health inventory (MHI), 1 study based on Hamilton depression rating scale (HDRS), 1 study based on the quick inventory of depressive symptomatology (QIDS), and 1 study based on the geriatric depression scale (GDS) had examined depression in veterans. The results of the metaanalysis showed that the prevalence of depression according to the statistical manual of mental disorders-IV (DSM-IV), the patient health questionnaire (PHQ), and beck depression inventory (BDI) was 15\% (\% 95 CI; 922\%), 21\% (\% 95 CI; 17-25\%), and 14\% (\% 95 CI; 9$21 \%$ ), respectively (Table 3 ).

The prevalence of depression in veteran military personnel in the three countries of the United States, 
Croatia and Canada was calculated and the results were reported in Table 3. The results of subgroup analysis showed that the majority of studies, the prevalence of which after meta-analysis was $20 \%$ (with a confidence interval of 18 to $22 \%$ ), to determine the prevalence of this outcome in veteran military personnel had been performed in the United States. The outcome prevalence in veteran military personnel in Canada and Croatia was 13 and $4 \%$, respectively. The rest of the studies had been individually conducted in only one country and because of their number, they could not be used for meta-analysis (Table 3).

\section{Publication bias, and meta-regression in studies related to veterans}

The results of the publication bias were shown in Fig. 2 for studies related to veterans. The results of the Eggers test presented that bias occurred in calculating the prevalence of depression in veterans (B: 8.95, SE: 0.54, P: 0.001) (Fig. 2). In meta-regression analysis, the effect of military age on prevalence was examined and analyzed, which showed that age did not have a significant effect on the prevalence of depression in military veterans.

\section{Prevalence of suicide in the military}

The results of this study demonstrated that 49 studies related to the prevalence of suicidal ideation in the military and 42 studies related to the prevalence of suicide attempts in the military were included in the metaanalysis. The sample size in studies related to suicidal ideation was 759,374 people, of whom a total of 20,065 individuals had suicidal ideation. However, the sample size in studies related to suicide attempts was equal to 438,890 people, of whom 5471 people had attempted suicide. The results of meta-analysis showed that the pooled prevalence of suicidal ideation in the entire military was 11\% (\% 95 CI; 10-13\%) (Fig. 3). The pooled prevalence of suicide attempts in all military was equal to the prevalence of suicidal ideation 11\% (\% $95 \mathrm{CI}$; 913\%) (Fig. 4).

To accurately estimate the prevalence of suicidal ideation in the military and to find the source of heterogeneity in the study, the subgroup analysis was performed based on whether the military person was serving or a veteran at that time, the study sampling method (random or convinces), the military service location, the statistical population of the study in terms of the presence of various diseases or being healthy, gender, and finally the tools used to measure suicide ideation and attempts. The results were shown in Table 4 . As can be seen from the results, the pooled prevalence of suicidal ideation in veterans was higher than that in active military $(14 \%$ vs. $10 \%)$. Suicidal ideation was also higher in women than men (Table 4). The pooled prevalence of suicidal ideation was higher in the air force (19\%) than that in the navy and the army (Table 4). In the military with substance use, the prevalence of suicidal ideation was $18 \%$ (\% 95 CI; 7-33\%), which was higher than one in the military consuming alcohol with a prevalence of $9 \%(\%$ $95 \mathrm{CI} ; 4-13 \%$ ) (Table 4). In studies that had used multiproblem screening inventory (MPSI) and the selfinjurious thoughts and behaviors interview (SITBI) to estimate suicidal ideation, the prevalence was 39\% (\% 95 CI; 36-41\%), and 36\% (\% 95 CI; 32-41\%), respectively, which was higher than those in studies that had used other tools to estimate the prevalence of suicidal ideation in the military (Table 4).

In terms of the prevalence of suicide attempts, servicemen serving in the air force were more likely to commit suicide than ones in the army (13\% vs. $12 \%)$. In the present analysis, the prevalence of suicide attempts in the navy was $54 \%$, but this was the result of a study with a sample size of 100 people that could not be trusted and compared with the prevalence of suicide attempts in other military (Table 4).

The prevalence of suicide attempts in militaries with substance use was 30\% (\% 95 CI; 23-36\%), which was higher than the prevalence of suicide attempts in nondrug-using military. Also, the prevalence of suicide attempts was $8 \%$ in militaries consuming alcohol (\% $95 \mathrm{CI}$; $7-10 \%)$ and in militaries with AIDS / HIV, it was equal to $5 \%$ (\% $95 \mathrm{CI}$; 4-8\%) (Table 4). Also, suicide attempts in female soldiers was more than that in male soldiers (21\% vs. $3 \%)$ (Table 4$)$.

The prevalence of suicide attempts was also analyzed based on the tools used in the studies. The results showed that after combining studies using SCID DSMIV diagnoses, beck scale for suicidal ideation-current (BSSI-C), multi-problem screening inventory (MPSI) and the suicidal behavior questionnaire revised (SBQ-R), the prevalence was 5\% (\% $95 \mathrm{CI} ; 3-7 \%$ ), 15\% (\% $95 \mathrm{CI} ; 10$ 22\%) 36\% (\% 95 CI; 33-38\%), 11\% (\% 95 CI; 1-49\%), respectively (Table 4).

The prevalence of suicidal ideation in the US military was $12 \%$ with a confidence interval of 10 to $14 \%$ while in the Canadian military, it was $7 \%$ with a confidence interval of 6 to $10 \%$. The prevalence of suicide attempts in the US military was also higher than that in the Canadian, Australian, British and Korean military (Table 4).

\section{Publication bias, and meta-regression in studies related to the spread of suicide ideation and attempts}

The results of the diffusion bias were shown in Fig. 5 . The results of the Eggers test represented that diffusion bias occurred in calculating the prevalence of suicidal ideation (B: 7.59, SE: 0.99, P: 0.001) and suicide attempts (B: 7.03, SE: 0.44, P: 0.001) in the military (Fig. 5). In meta-regression analysis, the effect of military age on 


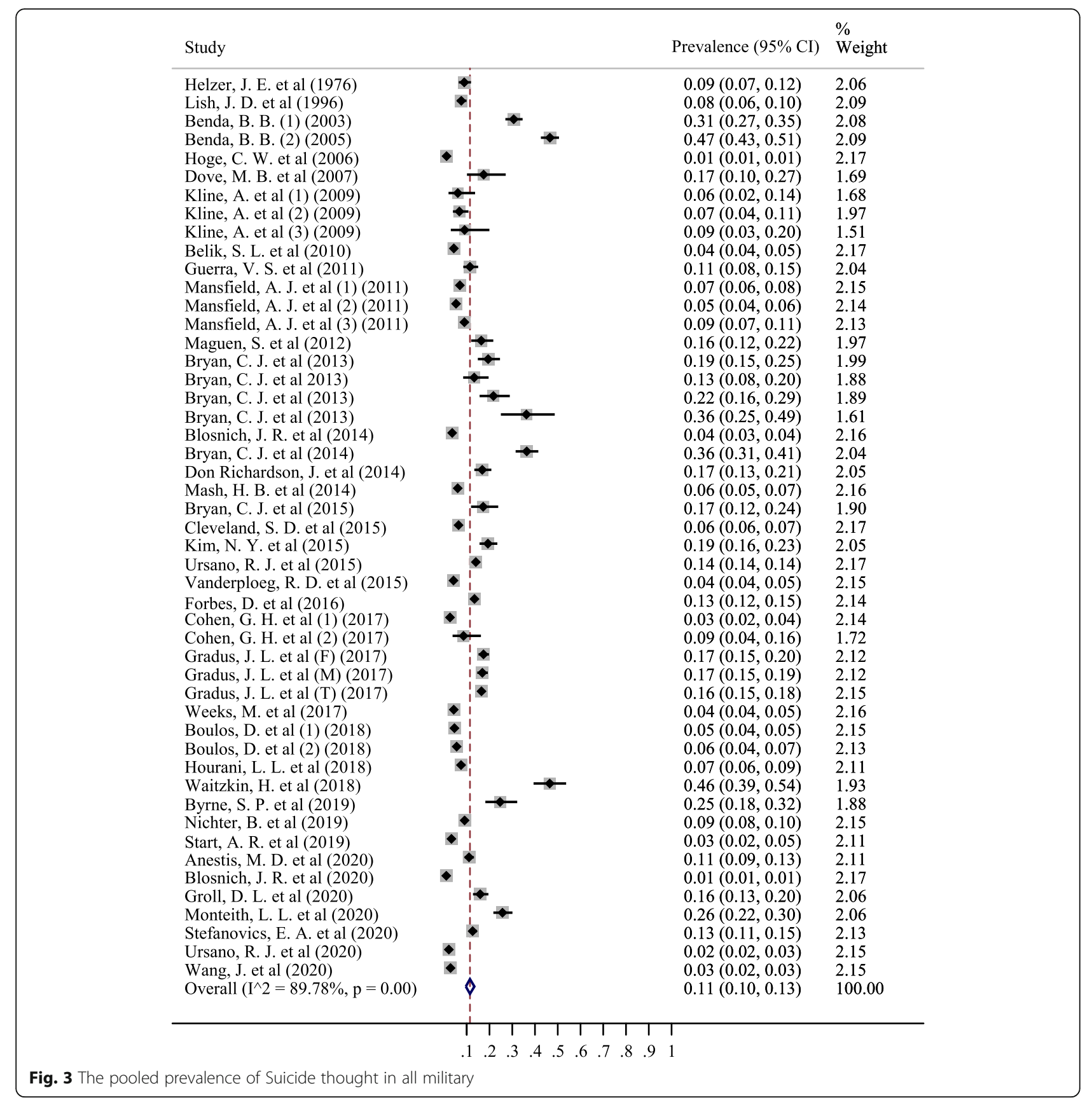

prevalence was examined and analyzed. The results showed that age did not have a significant effect on the prevalence of suicidal ideation and suicide attempts in the military.

\section{Discussion}

The present study was a systematic review and metaanalysis that showed that the pooled prevalence of depression in the active military was $23 \%$. According to the World Health Organization, the prevalence of depression in the general population is 15 to $20 \%$ [37, 38].
Therefore, it can be said that the prevalence of depression in the military community is higher than that in the general community. Feeling sad in unfavorable situations such as military situations and operational locations can be one of the reasons for the increase in the prevalence of depression or in some way the occurrence of depression and its symptoms in the military. This relationship indicates the existence of a relation between activity abnormalities, mood and thoughts with social or occupational environments [23, 39-42]. On the other hand, the military may not be very interested in their job and, 


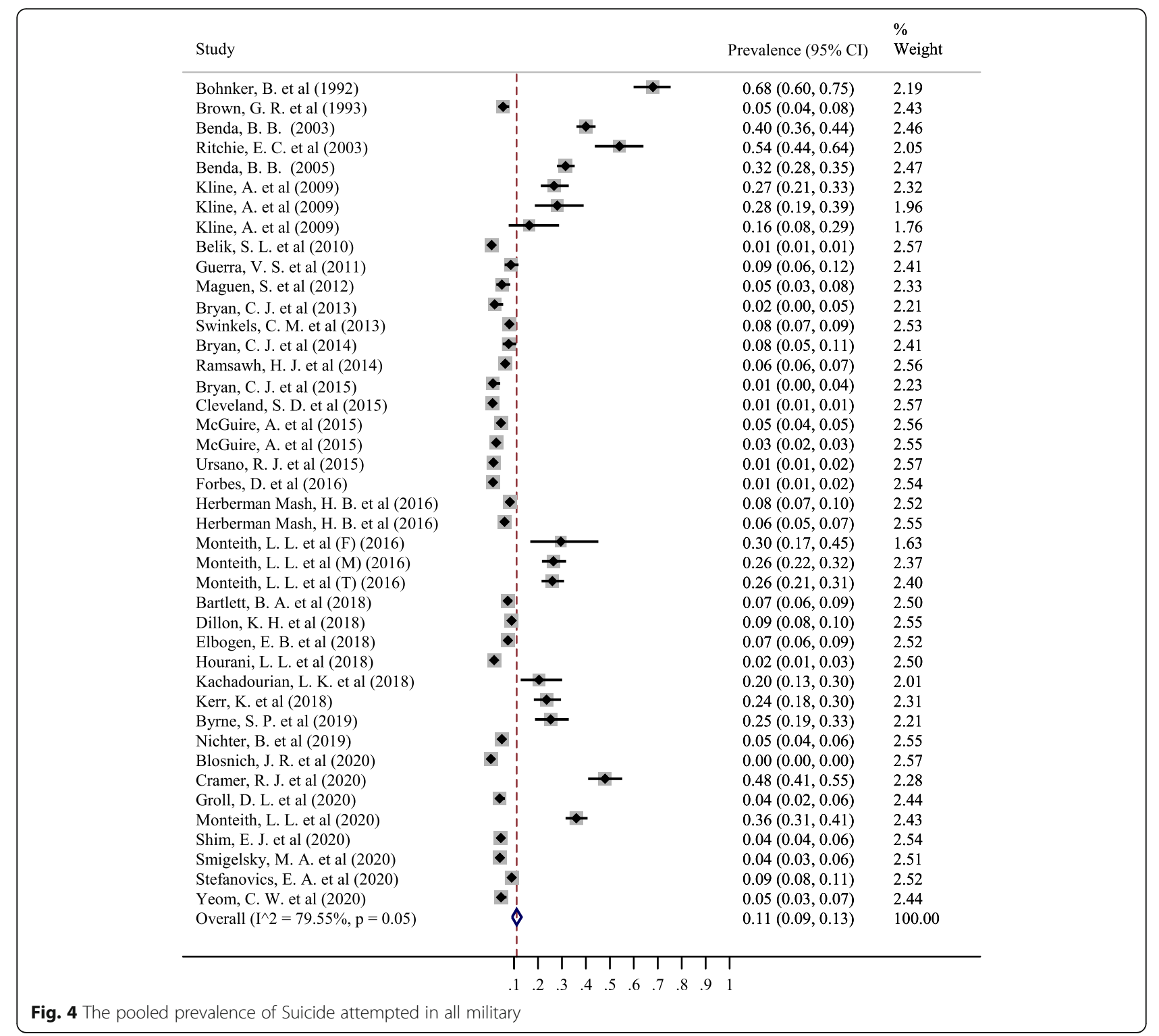

therefore, they have unpleasant moods and thoughts such as sadness, grief, despair and worry, which can make a military person prone to depression [43, 44]. Military personnel often suffer from disorders in sleep, nutrition, physical exertion, concentration, as well as anorexia, and weight changes due primarily to job sensitivity and confidential activities. The presence of these behaviors and emotions over time and their stability for a long time have a negative effect on the mood of these people and can expose a military person to depression $[24,45]$. In the present meta-analysis, the pooled prevalence of depression after combining studies in which the available sampling method had been used, was equal to $21 \%$ and after combining studies that had used random sampling method to collect their samples, the pooled prevalence of depression was equal to $26 \%$. In cross- sectional studies, the sampling method should be random in order to consider samples under investigation as a good representative of the target population. In studies that had selected this type of sampling, the pooled prevalence of depression was higher. On the other hand, the results of the subgroup analysis showed that the amount of heterogeneity after the analysis based on the sampling method has decreased, which indicated that different sampling methods in meta-analysis studies were one of the sources of heterogeneity in the total pooled prevalence in the active military.

The results of the present meta-analysis represented that the prevalence of depression was higher in active servicemen in the navy than in those in the air force and the army. The navy has more professional problems in terms of special professional missions, and more 
Table 4 The pooled estimate of prevalence of suicide in active duty and veteran military

\begin{tabular}{|c|c|c|c|c|c|c|c|}
\hline \multirow[t]{2}{*}{ Categories } & \multirow{2}{*}{$\begin{array}{l}\text { No. of } \\
\text { Studies } \\
\text { (Sample } \\
\text { Size) }\end{array}$} & \multirow{2}{*}{$\begin{array}{l}\text { Pooled } \\
\text { Prevalence } \\
\text { (\% 95 Cl) }\end{array}$} & \multicolumn{3}{|c|}{$\begin{array}{l}\text { Between studies heterogeneity } \\
\text { assessment (\%) }\end{array}$} & \multicolumn{2}{|c|}{$\begin{array}{l}\text { Between subgroups heterogeneity } \\
\text { assessment }(\%)\end{array}$} \\
\hline & & & $\overline{l^{2}}$ & $P_{\text {Heterogenity }}$ & $z$ & $\bar{Q}$ & $P_{\text {Heterogenity }}$ \\
\hline \multicolumn{8}{|c|}{ The prevalence of suicide thought in military } \\
\hline \multicolumn{8}{|l|}{ Military Statue } \\
\hline Active Duty & $31(424253)$ & $10 \%(7-13 \%)$ & $67.55 \%$ & 0.402 & 12.55 & 2.24 & 0.130 \\
\hline Veteran & $18(335121)$ & $14 \%(10-20 \%)$ & $69.77 \%$ & 0.329 & 9.59 & & \\
\hline \multicolumn{8}{|l|}{ Sampling Method } \\
\hline Convinces Sampling & 40 (151199) & $12 \%(10-15 \%)$ & $57.23 \%$ & 0.170 & 20.37 & 15.76 & 0.001 \\
\hline Random Sampling & $9(608175)$ & $7 \%(6-9 \%)$ & $74.31 \%$ & 0.059 & 17.83 & & \\
\hline \multicolumn{8}{|l|}{ Type of Forces } \\
\hline Air Forces & $2(441)$ & $19 \%(15-22 \%)$ & $78.99 \%$ & 0.041 & 17.36 & 30.05 & 0.001 \\
\hline Armed Forces & $23(434677)$ & $8 \%(5-11 \%)$ & $88.68 \%$ & 0.025 & 9.57 & & \\
\hline Marine Forces & $2(295715)$ & $1 \%(1-2 \%)$ & $93.86 \%$ & 0.0001 & 10.98 & & \\
\hline Military Forces & $22(28982)$ & $16 \%(12-21 \%)$ & $77.83 \%$ & 0.041 & 13.22 & & \\
\hline Population Healthy Forces & $42(757597)$ & $11 \%(9-13 \%)$ & $99.80 \%$ & 0.0001 & 20.07 & 1.74 & 0.420 \\
\hline Forces with HIV/AIDS & - & - & & - & - & & \\
\hline Forces with Alcohol Use & $1(93)$ & $9 \%(4-13 \%)$ & - & - & 4.45 & & \\
\hline Forces with Substance Use & $6(1684)$ & $18 \%(7-33 \%)$ & $97.74 \%$ & 0.0001 & 4.91 & & \\
\hline \multicolumn{8}{|l|}{ Gender } \\
\hline Total & $44(756218)$ & $11 \%(9-13 \%)$ & $88.72 \%$ & 0.0001 & 20.10 & 12.30 & 0.001 \\
\hline Male & $2(1532)$ & $14 \%(12-16 \%)$ & $90.00 \%$ & 0.0001 & 10.94 & & \\
\hline Female & $3(1624)$ & $20 \%(14-27 \%)$ & $75.22 \%$ & 0.017 & 28.78 & & \\
\hline \multicolumn{8}{|l|}{ Tools } \\
\hline BSSI-C Scale & $5(12775)$ & $11 \%(7-16 \%)$ & $67.96 \%$ & 0.052 & 8.98 & 24.84 & 0.001 \\
\hline SCRENNER Scale & $1(669)$ & $8 \%(6-10 \%)$ & - & - & 13.54 & & \\
\hline SCID DSM-IV Scale & $16(375640)$ & $7 \%(5-10 \%)$ & $69.80 \%$ & 0.049 & 10.23 & & \\
\hline MPSI Scale & $2(1225)$ & $39 \%(36-41 \%)$ & $55.21 \%$ & 0.077 & 15.52 & & \\
\hline PHQ Scale & $15(324540)$ & $9 \%(6-13 \%)$ & $53.01 \%$ & 0.850 & 9.61 & & \\
\hline SITBI Scale & $2(443)$ & $36 \%(32-41 \%)$ & $44.34 \%$ & 0.501 & 13.35 & & \\
\hline SBQ-R Scale & $7(5845)$ & $16 \%(14-18 \%)$ & $77.69 \%$ & 0.053 & 12.25 & & \\
\hline C-SSRS Scale & $1(38237)$ & $14 \%(12-18 \%)$ & - & - & 14.98 & & \\
\hline \multicolumn{8}{|l|}{ Country } \\
\hline USA & $42(707764)$ & $12 \%(10-14 \%)$ & $79.90 \%$ & 0.001 & 19.65 & 21.35 & 0.001 \\
\hline Canada & $6(49560)$ & $7 \%(6-10 \%)$ & $76.92 \%$ & 0.001 & 14.19 & & \\
\hline \multicolumn{8}{|c|}{ The prevalence of suicide attempted in military } \\
\hline \multicolumn{8}{|l|}{ Military Statue } \\
\hline Active Duty & 19 (98426) & $8 \%(6-10 \%)$ & $50.18 \%$ & 0.497 & 12.14 & 10.13 & 0.001 \\
\hline Veteran & $23(340464)$ & $15 \%(11-19 \%)$ & $69.80 \%$ & 0.122 & 12.59 & & \\
\hline \multicolumn{8}{|l|}{ Sampling Method } \\
\hline Convinces Sampling & 35 (133437) & $11 \%(9-13 \%)$ & $77.78 \%$ & 0.059 & 16.11 & 0.30 & 0.660 \\
\hline Random Sampling & 7 (305453) & $13 \%(7-20 \%)$ & $64.26 \%$ & 0.051 & 6.47 & & \\
\hline \multicolumn{8}{|l|}{ Type of Forces } \\
\hline Air Forces & $4(4851)$ & $13 \%(1-35 \%)$ & $79.99 \%$ & 0.047 & 2.54 & & \\
\hline Armed Forces & $23(121644)$ & $12 \%(9-15 \%)$ & $76.44 \%$ & 0.044 & 14.50 & 1.27 & 0.260 \\
\hline
\end{tabular}


Table 4 The pooled estimate of prevalence of suicide in active duty and veteran military (Continued)

\begin{tabular}{|c|c|c|c|c|c|c|c|}
\hline \multirow[t]{2}{*}{ Categories } & \multirow{2}{*}{$\begin{array}{l}\text { No. of } \\
\text { Studies } \\
\text { (Sample } \\
\text { Size) }\end{array}$} & \multirow{2}{*}{$\begin{array}{l}\text { Pooled } \\
\text { Prevalence } \\
(\% 95 \mathrm{Cl})\end{array}$} & \multicolumn{3}{|c|}{$\begin{array}{l}\text { Between studies heterogeneity } \\
\text { assessment (\%) }\end{array}$} & \multicolumn{2}{|c|}{$\begin{array}{l}\text { Between subgroups heterogeneity } \\
\text { assessment (\%) }\end{array}$} \\
\hline & & & $\overline{P^{2}}$ & $P_{\text {Heterogenity }}$ & $z$ & $\bar{Q}$ & $P_{\text {Heterogenity }}$ \\
\hline Marine Forces & $1(100)$ & $54 \%(44-64 \%)$ & - & - & 15.54 & & \\
\hline Military Forces & $14(312295)$ & $8 \%(4-12 \%)$ & $74.77 \%$ & 0.034 & 6.82 & & \\
\hline \multicolumn{8}{|l|}{ Population } \\
\hline Healthy Forces & $35(435640)$ & $9 \%(8-11 \%)$ & $99.09 \%$ & 0.0001 & 18.52 & 84.99 & 0.001 \\
\hline Forces with HIV/AIDS & $1(442)$ & $5 \%(4-8 \%)$ & - & - & 19.33 & & \\
\hline Forces with Alcohol Use & $1(1210)$ & $8 \%(7-10 \%)$ & - & - & 14.59 & & \\
\hline Forces with Substance Use & $5(1598)$ & $30 \%(23-36 \%)$ & $87.44 \%$ & 0.0001 & 8.99 & & \\
\hline Forces with HCV & - & - & - & - & - & & \\
\hline \multicolumn{8}{|l|}{ Gender } \\
\hline Total & $37(429113)$ & $11 \%(9-13 \%)$ & $92.88 \%$ & 0.0001 & 9.04 & 9.56 & 0.001 \\
\hline Male & $2(4533)$ & $3 \%(2-4 \%)$ & $95.91 \%$ & 0.0001 & 2.49 & & \\
\hline Female & $3(5244)$ & $21 \%(1-53 \%)$ & $98.49 \%$ & 0.0001 & 10.75 & & \\
\hline \multicolumn{8}{|l|}{ Tools } \\
\hline BSSI-C Scale & $6(9800)$ & $15 \%(10-22 \%)$ & $66.33 \%$ & 0.050 & 9.04 & 35.33 & 0.001 \\
\hline Checklist & $6(6882)$ & $11 \%(5-20 \%)$ & $78.31 \%$ & 0.049 & 5.25 & & \\
\hline SCID DSM-IV Scale & $12(373059)$ & $5 \%(3-7 \%)$ & $61.99 \%$ & 0.041 & 11.15 & & \\
\hline MPSI Scale & $2(1225)$ & $36 \%(33-38 \%)$ & $55.99 \%$ & 0.055 & 43.39 & & \\
\hline PHQ Scale & $4(4675)$ & $9 \%(5-15 \%)$ & $70.05 \%$ & 0.039 & 6.54 & & \\
\hline MINI-Plus Scale & $2(2417)$ & $4 \%(4-5 \%)$ & $60.44 \%$ & 0.041 & 19.36 & & \\
\hline SBQ-R Scale & $3(526)$ & $11 \%(1-49 \%)$ & $69.01 \%$ & 0.050 & 1.42 & & \\
\hline SITBI Scale & $1(374)$ & $8 \%(5-11 \%)$ & - & - & 10.00 & & \\
\hline C-SSRS Scale & $2(38330)$ & $1 \%(1-2 \%)$ & $62.99 \%$ & 0.034 & 32.48 & & \\
\hline NR & $3(692)$ & $22 \%(2-53 \%)$ & $79.90 \%$ & 0.045 & 2.76 & & \\
\hline \multicolumn{8}{|l|}{ Country } \\
\hline Australia & $2(2279)$ & $2 \%(1-4 \%)$ & $0.0 \%$ & 0.777 & 12.13 & & \\
\hline Canada & $2(37606)$ & $1 \%(1-2 \%)$ & $0.0 \%$ & 0.832 & 25.28 & & \\
\hline Korea & $2(2417)$ & $4 \%(3-5 \%)$ & $0.0 \%$ & 0.489 & 19.36 & 19.75 & 0.001 \\
\hline United Kingdom & $3(10492)$ & $5 \%(2-8 \%)$ & $0.0 \%$ & 0.880 & 6.64 & & \\
\hline USA & 33 (386096) & $14 \%(11-16 \%)$ & $60.98 \%$ & 0.066 & 16.97 & & \\
\hline
\end{tabular}

Beck Scale for Suicidal Ideation-Current (BSSI-C), Brief self-report questionnaire (SCRENNER), SCID DSM-IV Diagnoses, Multi-Problem Screening Inventory (MPSI), The 4-item Suicidal Behaviors Questionnaire-Short Form (SBQ-SF), The Patient Health Questionnaire(PHQ), The Self-Injurious Thoughts and Behaviors Interview (SITBI), The Suicidal Behaviors Questionnaire Revised (SBQ-R), The Columbia Suicidal Severity Rating Scale (C-SSRS), the Mini International Neuropsychiatric Interview Plus (MINI-Plus)

psychological problems than the army and the air Force. Job-related stress, complex missions, strict rules, the possibility of injury, disability, captivity and even death are some of the issues that increase the likelihood of depression in these soldiers compared to others [46, 47]. A person's psychological capacity includes a person's ability to cope with the expectations and difficulties of everyday life. High psychological capacity allows a person to maintain his/her life at the desired psychological level and crystallize this ability in the form of adaptive behaviors, effective and positive actions for himself/herself. The role of psychological capacity in promoting health and well-being in all three aspects of physical, mental and social is very important. This importance becomes even more apparent when the problem becomes behavioral. In such a case, the person is not strong enough when faced with psychological pressures and obstacles in life, and as a result, his/her inappropriate behavior will be the source of all suffering and failure [48, 49]. Therefore, addressing various psychological aspects, quality of life and social relations of the military, especially the navy, in order to properly understand the conditions of these people and their families can be useful to strengthen and enhance their military capabilities and 


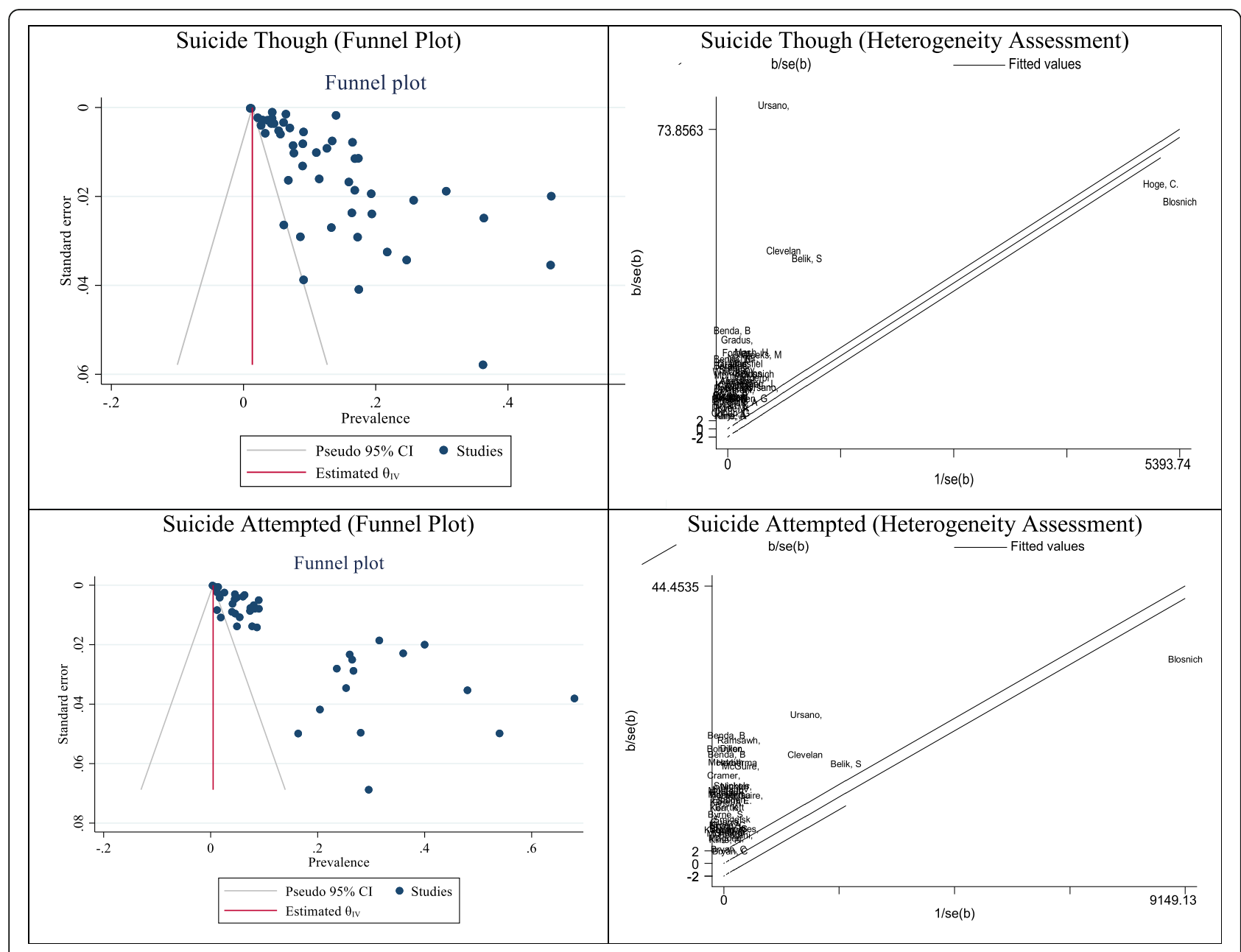

Fig. 5 Results of Publication bias and Heterogeneity in pooled prevalence of Suicide though and attempted in all military

efficiency. Other reasons for the increasing prevalence of depression in the navy include family problems [50]. Over the years, research has shown that the family plays an important role in providing function and activity to individuals. Having a healthy society depends on having strong families in the society. Navy families often suffer from the stress of being away from a normal life, living in unfamiliar environments, and experiencing life outside their homelands. These may cause problems within the family, which ultimately reduce the ability of the navy and cause psychological problems such as depression $[51,52]$.

The stress of military jobs has major and significant consequences for the family environment. Psychological disorders between military families have been reported between 3 to 15\% depending on the disorder type, while they have been reported paranoid disorders, obsessivecompulsive disorders, depression, interpersonal relationships, physical problems, and aggression, respectively [52]. According to research, it has been shown that the prevalence of these disorders in military families was higher than that in other families in the society. Factors such as workplace stress, sensitive and critical situations, high job responsibilities, job stress, unwanted relocation, problems in the family and home, lack of confidence in individual abilities, mental fatigue caused by hard work, thinking the possibility of death are some of the depression and mental distress causes in the military and their families [53, 54]. In a study entitled Environment, Lifestyle and Psychological Factors in the Health and Welfare of Military Families, the results showed that the psychological factors resulting from military missions were divided into 5 stages which included the stage before deployment, deployment, return, reinforcement and re-deployment, respectively. Military personnel and their families also experienced different psychological difficulties before, during, or after deployment to different missions. These experiences brought them many psychological norms that varied with different variables such as the location of the mission (in terms of the possibility of military conflict with hostile forces), duration of deployment, number of deployments, time between 
deployments, military responsibility, and the difficulty of working conditions of individuals at the time of deployment [55-57]. The same factors may lead military personnel to use drugs, and alcohol [58]. In the present meta-analysis, the prevalence of depression in the active military drug users was $37 \%$ and in the military alcohol users was $29 \%$. Drug, and alcohol abuse can be a contributing factor to depression or other mental disorders in the military. Excessive alcohol abuse in the US military has resulted in significant financial losses. Data from 2006 showed that excessive alcohol consumption annually cost the US military 1.12 billion dollars [59, 60]. In a large survey study by Bray and Hourani, the results demonstrated that the prevalence of alcohol use in the US military was 15 to $20 \%$ [61]. Also, in terms of gender, this prevalence was different and in men, alcohol consumption was 3.5 times more than that in military women. The results of studies have shown that the prevalence of alcohol and drug use in the Navy was higher than that in the Air Force, which might be related to the high prevalence of depression in the navy [6264]. Alcohol and substance abuse occur more frequently in war veterans. A study by Milliken and colleagues in a population-based study found that 12 to $15 \%$ of veterans experienced alcohol and substance abuse after 3 to 6 months of returning from war, which put them at risk of depression [65-67]. In the present meta-analysis, the overall pooled prevalence of depression in veterans was $20 \%$. However, in studies that had used random sampling to collect samples, the prevalence was $22 \%$.

The prevalence of depression was $15 \%$ in active HIV-positive servicemen and $16 \%$ in HIV-positive veterans. These military personnel, of course, suffered from depression and other mental disorders due to the existence of the disease and its difficult conditions in the society. The prevalence of depression in veterans with hepatitis C was $29 \%$. It was noteworthy that the amount of heterogeneity during the subgroup analysis based on the healthy and unhealthy military population did not significantly decrease compared to the overall prevalence of heterogeneity, which indicated the lack of the inclusion effect of soldiers with various diseases, and healthy soldiers on the amount of heterogeneity in studies. In other words, this factor could not be a source of heterogeneity when estimating the overall prevalence of depression. However, as shown in Table 4, the type of sampling (random or available), location and place of service (the air, naval or army), and various tools for measuring the prevalence of depression were the main sources of heterogeneity when estimating general depression in the military because the amount and percentage of heterogeneity had significantly decreased when performing subgroups based on these variables.
The prevalence of suicidal ideation in the present meta-analysis in the military was equal to the prevalence of suicide attempts in the entire military. Suicidal ideation was also more common in women than in military men. According to studies conducted in the world, the prevalence of suicide and its thoughts in the military had a range from 5.8 to $28.4 \%$, which in the present meta-analysis study was exactly equal to $11 \%$. In the study of Farsi et al., the results showed that with increasing scores of depression, the possibility of self-harm and suicide in the military increased [68]. In the study by Hossieni et al., The prevalence of depressive disorders in military personnel who had attempted suicide was 0.7 to $1.3 \%$ [69]. The prevalence of suicidal ideation was higher in Air Force servicemen than that in Navy and Land Force servicemen. The prevalence of suicidal ideation was $18 \%$ in the military using drug, which was higher than that in the military using alcohol. Also, the prevalence of suicide attempts in drug-using military was higher than the prevalence of suicide attempts in non-drug-using military. The results of the present meta-analysis showed that the use of drugs, alcohol and diseases such as HIV and HCV could be a predisposing factor in the development of mental disorders and the development of suicidal ideation and suicide attempts in the military. In addition, there were more thoughts and attempts to commit suicide in veterans than in active and serving soldiers. One of the effective reasons for the existence of suicidal ideation and attempts in the veterans was the lack of combat and other physical activities, living at home, consuming drugs and alcohol. The results of the present meta-analysis represented that the prevalence of suicidal ideation and attempts in military personnel using drugs were equal to 18 and $30 \%$, respectively.

Regarding the prevalence of suicidal ideation and attempts, the results of the subgroup analysis showed that the use of different tools in determining the prevalence of suicidal ideation in the military in meta-analysis studies, different sampling methods (available or random sampling), and the type of servicemen included in the study (in-service or veterans) were among the most important factors in creating heterogeneity in determining the pooled prevalence of suicidal ideation and attempts in the military after completing the entire study. The subgroup analysis was based on different countries, but most studies had been conducted in the United States. The following subgroup results showed that the prevalence of depression in the US active military was $21 \%$ (with a confidence interval of 17 to $25 \%$ ) while the prevalence of depression in the Thailand and British military was higher than that in other countries, which was 39 and 
$30 \%$, respectively. The prevalence of depression was higher in retired US troops than that in retired Canadian and Croatian troops. Also, the prevalence of suicidal ideation in the US military was higher than that in the Canadian, Australian, British and Korean militaries. In this analysis, the amount of heterogeneity significantly decreased in different subgroups, which indicated the role of different cultures, different military methods for training soldiers, and different military environments in various countries as the sources of heterogeneity.

In this meta-analysis, the finding of articles published from January 1990 to December 2020 was analyzed. Articles on suicide or depression in the military have been published in PubMed since 1966. But, these types of studies did not have the appropriate structure of original or cross-sectional studies (which were the main studies included in this meta-analysis). In addition, studies before 1990 did not have a suitable sample size to be able to enter the present meta-analysis. Finally, articles from 1990 to 2020 were considered to avoid creating too much heterogeneity and bias in the results. In this study, it was decided to determine the exact prevalence because meta-analysis of prevalence gives the reader and health policy makers better interpretations than the average, and this value is more tangible for health policy makers. Also, estimating the prevalence of depression and suicide can be effective and useful in estimating the burden of these diseases and in planning health programs for the military of the world.

The present meta-analysis study was the first systematic review and meta-analysis study to determine the prevalence of depressive and suicidal disorders in the entire military worldwide. Also, the exact prevalence of these disorders in the military had not been reported and this research determined the overall pooled prevalence of depression and suicidal ideation or attempts. On the other hand, the sample size in the present metaanalysis subgroup was very significant, which made the estimated prevalence in each subgroup very reliable. Other benefits of this study included determining the prevalence of depressive disorders and suicide in military personnel in various sectors, such as the navy, air, and army forces. One of the limitations of the present study was the lack of sufficient number of studies and sample sizes to determine the prevalence of depressive and suicidal disorders in servicemen with hepatitis $\mathrm{C}$ or other diseases. For future research, the issue of social classes, religion, and income levels need to be considered to determine the prevalence of mental disorders in the military. Also, studies on how to carry out preventive interventions, and their cost-effectiveness need to be done in order to determine effective and useful interventions in the military to prevent suicide and depression.

\section{Conclusion}

The present study showed that the prevalence of depression and suicide (thoughts and actions) was high in the military, especially in the navy and air forces, and this prevalence was more significant. On the other hand, substance and alcohol consumption were factors that increased the prevalence of depression and ultimately led to suicide in the military. Therefore, it is necessary to develop and design training and intervention programs in order to train and increase the awareness of the military, especially veterans, in order to prevent the occurrence of suicide and mental disorders such as depression. Considering the prevalence of depression and suicide in the military consuming drugs and alcohol in the present meta-analysis study, it is necessary to implement screening and follow-up measures to identify, and prevent these two disorders (drug and alcohol consumption) in the military.

\section{Abbreviations}

Cl: Confidence Interval; EMBASE: Excerpta Medica dataBASE; NOS: NewcastleOttawa Scale; MOOSE: The Meta-Analyses of Observational Studies in Epidemiology; PRISMA: Preferred Reporting Items for Systematic Reviews and Meta-analyses; WHO: World Health Organization; DSM-IV: The diagnostic and statistical manual of mental disorders-IV; PHQ: The patient health questionnaire; SDS: The Zung self-tool report depression scale; BDI: The beck depression inventory; CES-D: The center for epidemiological studies depression; HDRS: Hamilton depression rating scale; MHI: Mental health inventory; QIDS: The quick inventory of depressive symptomatology; GDS: The geriatric depression scale; SCID DSM-IV: Structured Clinical Interview for DSM Disorders; BSSI-C: Beck scale for suicidal ideation-current; MPSI: Multi-problem screening inventory; SBQ-R: The suicidal behavior questionnaire revised; SCRENNER: Brief self-report questionnaire; MPSI: MultiProblem Screening Inventory; SBQ-SF: The 4-item Suicidal Behaviors

Questionnaire-Short Form; SITBI: The Self-Injurious Thoughts and Behaviors Interview; SBQ-R: The Suicidal Behaviors Questionnaire Revised; C-SSRS: The Columbia Suicidal Severity Rating Scale; MINI-Plus: The Mini International Neuropsychiatric Interview Plus; BSI: The Brief Symptom Inventory; HADS: The Hospital Anxiety and Depression Scale; GDS: Geriatric Depression Scale; QIDS: Quick Inventory of Depressive Symptomatology

\section{Supplementary Information}

The online version contains supplementary material available at https://doi. org/10.1186/s12888-021-03526-2.

Additional file 1. The search syntax in PubMed and Embase.

\section{Acknowledgments}

Not applicable.

\section{Authors' contributions}

$\mathrm{YM}, \mathrm{BD}$, and MS conceptualized the idea for this review, formulated the review question, and objectives. All authors contributed equally to the formulation of the development of the search strategy, conducting the searches, data extraction, data analysis/interpretation, and writing the manuscript. All authors read and approved the final manuscript.

\section{Funding}

None.

Availability of data and materials

Data is available and it can be accessed from the corresponding author with reasonable inquiry. 


\section{Declarations}

\section{Ethics approval and consent to participate}

Not applicable because no primary data were collected.

\section{Consent for publication}

Not applicable.

\section{Competing interests}

The authors declare that they have no competing interests.

\section{Author details}

${ }^{1}$ Health Research Center, Life Style Institute, Baqiyatallah University of Medical Sciences, Tehran, Iran. ${ }^{2}$ Social Determinants of Health Research Center, Research Institute for Health Development, Kurdistan University of Medical Sciences, Sanandaj, Iran. ${ }^{3}$ Behavioral Sciences Research Center, Life style institute, Baqiyatallah University of Medical Sciences, Tehran, Iran.

Received: 24 February 2021 Accepted: 8 October 2021

Published online: 15 October 2021

\section{References}

1. Wise T, Dolan RJ. Associations between aversive learning processes and transdiagnostic psychiatric symptoms in a general population sample. Nat Commun. 2020;11(1):4179. https://doi.org/10.1038/s41467-020-17977-w.

2. Best MW, Law H, Pyle M, Morrison AP. Relationships between psychiatric symptoms, functioning and personal recovery in psychosis. Schizophr Res. 2020;223:112-8. https://doi.org/10.1016/j.schres.2020.06.026.

3. Foxx $\mathrm{CL}$, et al. Effects of immunization with the soil-derived bacterium mycobacterium vaccae on stress coping behaviors and cognitive performance in a "two hit" stressor model. Front Physiol. 2020;11:524833.

4. Mota N, et al. Course and Predictors of Posttraumatic Stress Disorder in the Canadian Armed Forces: A Nationally Representative, 16-Year Follow-up Study: Cours et prédicteurs du trouble de stress post-traumatique dans les Forces armées canadiennes: une étude de suivi de 16 ans nationalement représentative. Can J Psychiatr. 2021;70(6):743-72. https://doi.org/10.1177/ 0706743721989167.

5. Sullivan KS, et al. Mental health outcomes associated with profiles of risk and resilience among U.S. Army spouses. J Fam Psychol. 2021;35(1):33-43. https://doi.org/10.1037/fam0000702.

6. Marini CM, Fiori KL, Wilmoth JM, Pless Kaiser A, Martire LM. Psychological adjustment of aging Vietnam veterans: the role of social network ties in reengaging with wartime memories. Gerontology. 2020;66(2):138-48. https://doi.org/10.1159/000502340.

7. Vogt DS, Tyrell FA, Bramande EA, Nillni YI, Taverna EC, Finley EP, et al. U.S. military Veterans' health and well-being in the first year after service. Am J Prev Med. 2020;58(3):352-60. https://doi.org/10.1016/j.amepre.2019.10.016.

8. Herzog S, Fogle BM, Harpaz-Rotem I, Tsai J, Pietrzak RH. Dissociative symptoms in a nationally representative sample of trauma-exposed U.S. military veterans: prevalence, comorbidities, and suicidality. J Affect Disord. 2020;272:138-45. https://doi.org/10.1016/j.jad.2020.03.177.

9. Brockelmeyer MC. The impact of military culture on veterans entering the private workforce: a qualitative ethnographic study. Northcentral University; 2020.

10. Wilcove GL, Schwerin MJ, Wolosin DG. An exploratory model of quality of life in the US navy. Mil Psychol. 2003;15(2):133-52. https://doi.org/10.1207/ S15327876MP1502_3.

11. Hindelang RL, Schwerin MJ, Farmer WL. Quality of life (QOL) in the US marine corps: the validation of a QOL model for predicting reenlistment intentions. Mil Psychol. 2004;16(2):115-34. https://doi.org/10.1207/S1532 7876MP1602_3.

12. Sticha PJ, et al. Personnel tempo: definition, measurement, and effects on retention, readiness, and quality of life. Alexandria: Human Resources Research Organization; 1999.

13. Levina $M$, Krieger E. Interrelation and interdependence of attitudes of choice of life path and propensity to deviant behavior in military personnel. Psychol Educ Stud. 2020;12(3):137-51. https://doi.org/10.17759/psyedu.202 0120309.

14. Dunlap SL, Holloway IW, Pickering CE, Tzen M, Goldbach JT, Castro CA. Support for transgender military service from active duty United States military personnel. Sex Res Social Policy. 2021;18(1):137-43. https://doi.org/1 0.1007/s13178-020-00437-X.
15. Sinnott J, Rabin JS. Psychological Mechanisms Underlying the Populist Threat to Democracy. The Psychology of Political Behavior in a Time of Change. 2019;24:473-527. https://doi.org/10.1007/978-3-030-38270-4_21.

16. Reyes ME, et al. Self-esteem and optimism as predictors of resilience among selected Filipino active duty military personnel in military camps. J Posit School Psychol. 2020;4(1):15-25.

17. Moody RL, Savarese E, Gurung S, Rendina HJ, Parsons JT. The mediating role of psychological distress in the association between harassment and alcohol use among lesbian, gay, and bisexual military personnel. Subst Use Misuse. 2020; 55(12):2055-63. https://doi.org/10.1080/10826084.2020.1790007.

18. Van Den Berk-Clark C, et al. The impact of hazardous alcohol use on behavioral healthcare utilization among National Guard service members. Subst Use Misuse. 2016;51(5):625-36. https://doi.org/10.3109/10826084.201 5.1133644.

19. Kelley ML, Milletich RJ, Hollis BF, Veprinsky A, Robbins AT, Snell AK. Social support and relationship satisfaction as moderators of the stress-moodalcohol link association in US navy members. J Nerv Ment Dis. 2017;205(2): 99-105. https://doi.org/10.1097/NMD.0000000000000555.

20. Coronges KA, Miller KA, Tamayo Cl, Ender MG. A network evaluation of attitudes toward gays and lesbians among US military cadets. J Homosex. 2013;60(11):1557-80. https://doi.org/10.1080/00918369.2013.824322.

21. Cadigan JM, Klanecky AK, Martens MP. An examination of alcohol risk profiles and co-occurring mental health symptoms among OEF/OIF veterans. Addict Behav. 2017;70:54-60. https://doi.org/10.1016/j.addbeh.201 7.02.009.

22. Yeom CW, Oh GH, Jung S, Moon JY, Son KL, Kim WH, et al. Prevalence and comorbidities of adult adhd in male military conscripts in Korea: results of an epidemiological survey of mental health in korean military service. Psychiatry Res. 2020;293:113401. https:/doi.org/10.1016/.jpsychres.2020.113401.

23. Wang J, Ursano RJ, Gifford RK, Dinh H, Faroog S, Broshek CE, et al. Mental health and suicidality in separating US reserve and National Guard personnel. Psychiatry. 2020;83(2):166-75. https://doi.org/10.1080/00332747.2 020.1715162 .

24. Thériault FL, Gardner W, Momoli F, Garber BG, Kingsbury M, Clayborne Z, et al. Mental health service use in depressed military personnel: a systematic review. Mil Med. 2020;185(7-8):e1255-62. https:/doi.org/10.1093/milmed/usaa015.

25. Smith BM, Twohy AJ, Smith GS. Psychological inflexibility and intolerance of uncertainty moderate the relationship between social isolation and mental health outcomes during COVID-19. J Contextual Behav Sci. 2020;18:162-74. https://doi.org/10.1016/j.jcbs.2020.09.005.

26. Presti G, Dal Lago B, Fattori A, Mioli G, Moderato P, Sciaretta L, et al. Mental health support to staff in a major hospital in Milan (Italy) during the COVID19 pandemic: a framework of actions. Gen Psychiatr. 2020;33(4):e100244. https://doi.org/10.1136/gpsych-2020-100244

27. McCracken LM, Badinlou F, Buhrman M, Brocki KC. Psychological impact of COVID-19 in the Swedish population: Depression, anxiety, and insomnia and their associations to risk and vulnerability factors. European Psychiatry. 2020; 63(1):e81. https://doi.org/10.1192/j.eurpsy.2020.81.

28. Stroup DF, Berlin JA, Morton SC, Olkin I, Williamson GD, Rennie D, et al. Metaanalysis of observational studies in epidemiology: a proposal for reporting. Metaanalysis of observational studies in epidemiology (MOOSE) group. Jama. 2000; 283(15):2008-12. https:/doi.org/10.1001/jama.283.15.2008.

29. Moher D, Liberati A, Tetzlaff J, Altman DG, PRISMA Group. Preferred reporting items for systematic reviews and meta-analyses: the PRISMA statement. Int I Surg. 2010; 8(5):336-41. https://doi.org/10.1016/.i.jsu.2010.02.007.

30. Moher D, Liberati A, Tetzlaff J, Altman DG, The PRISMA Group. Preferred reporting items for systematic reviews and meta-analyses: the PRISMA statement. PLoS Med. 2009;6(7):e1000097. https://doi.org/10.1371/journal.pmed.1000097.

31. Stang A. Critical evaluation of the Newcastle-Ottawa scale for the assessment of the quality of nonrandomized studies in meta-analyses. Eur J Epidemiol. 2010;25(9):603-5. https://doi.org/10.1007/s10654-010-9491-z.

32. Moskalewicz A, Oremus M. No clear choice between Newcastle-Ottawa Scale and Appraisal Tool for Cross-Sectional Studies to assess methodological quality in cross-sectional studies of health-related quality of life andbreast cancer. J clin Epidemiology. 2020;120:94-103. https://doi.org/1 0.1016/j.jclinepi.2019.12.013.

33. Higgins JP, Thompson SG. Quantifying heterogeneity in a meta-analysis. Stat Med. 2002;21(11):1539-58. https://doi.org/10.1002/sim.1186.

34. Higgins JP, Thompson SG, Deeks JJ, Altman DG. Measuring inconsistency in meta-analyses. Bmj. 2003;327(7414):557-60. https://doi.org/10.1136/bmj.327. 7414.557. 
35. Higgins JP, et al. Cochrane handbook for systematic reviews of interventions: Wiley; 2019.

36. Cumpston M, Li T, Page MJ, Chandler J, Welch VA, Higgins JPT, et al. Updated guidance for trusted systematic reviews: a new edition of the Cochrane handbook for systematic reviews of interventions. Cochrane Database Syst Rev. 2019;10:ED000142. https://doi.org/10.1002/14651858. ED000142.

37. Zandi A, et al. Abundance of depression, anxiety and stress in militant nurses. J Mil Med. 2011;13(2):103-8.

38. Mohammadi $\mathrm{M}$, et al. Prevalence of depression in nurses working in Iranian hospitals: a systematic review and Meta-analysis. Int J Med Rev. 2020;7(1): 32-8.

39. Groll DL, Ricciardelli R, Carleton RN, Anderson G, Cramm H. A cross-sectional study of the relationship between previous military experience and mental health disorders in currently serving public safety personnel in Canada. Can J Psychiatry. 2020;65(5):330-7. https://doi.org/10.1177/0706743719895341.

40. Rowan AB, Travis WJ, Richardson CB, Adams TR. Military mental health personnel deployment survey: a secondary analysis. Mil Med. 2020;185(3-4): e340-6. https://doi.org/10.1093/milmed/usz275.

41. Rugo KF, Tabares JV, Crowell SE, Baucom BR, Rudd MD, Bryan CJ. The role of depression and suicidal cognitions as contributors to suicide risk among active duty soldiers reporting symptoms of posttraumatic stress disorder. J Affect Disord. 2020;265:333-41. https://doi.org/10.1016/j.jad.2020.01.095.

42. Sadler N, Forbes D, O'Donnell M. Mental health, well-being, and suicidality following separation from the military: advancing research and practice. Psychiatry. 2020;83(2):176-8. https://doi.org/10.1080/00332747.2020.1767991.

43. Hu Y, Chu X, Urosevich TG, Hoffman SN, Kirchner HL, Adams RE, et al. Predictors of current DSM-5 PTSD diagnosis and symptom severity among deployed veterans: significance of predisposition, stress exposure, and genetics. Neuropsychiatr Dis Treat. 2020;16:43-54. https://doi.org/10.2147/ NDT.S228802.

44. Huang J, Li X, He W, Wan Y, Bao H, Xu Y, et al. Formation environment and development models for the lower Cambrian source rocks of the southern North China plate, China. ACS omega. 2020;5(14):8001-11. https://doi.org/1 0.1021/acsomega.0c00077.

45. Sheriff RS, et al. Childhood trauma and childhood mental disorder in military and employed civilian men. J Nerv Ment Dis. 2020;208(1):13-20. https://doi.org/10.1097/NMD.0000000000001072

46. Thériault FL, Hawes RA, Garber BG, Momoli F, Gardner W, Zamorski MA, et al. Incidence of major depression diagnoses in the Canadian Armed Forces: longitudinal analysis of clinical and health administrative data. Soc Psychiatry Psychiatr Epidemiol. 2020;55(5):581-8. https://doi.org/10.1007/ s00127-019-01754-2

47. Kim Halford W. The future of couple relationship education: suggestions on how it can make a difference. Fam Relat. 2004;53(5):559-66. https://doi. org/10.1111/j.0197-6664.2004.00065.x.

48. Busby DM, Ivey DC, Harris SM, Ates C. Self-directed, therapist-directed, and assessment-based interventions for premarital couples. Fam Relat. 2007; 56(3):279-90. https://doi.org/10.1111/j.1741-3729.2007.00459.x.

49. Ooms $T$, Wilson $P$. The challenges of offering relationship and marriage education to low-income populations. Fam Relat. 2004;53(5):440-7. https:// doi.org/10.1111/j.0197-6664.2004.00052.x.

50. Živić B, et al. Post-traumatic stress disorder psychotic subtype or comorbid psychotic disorder and evaluation of military service ability. Vojnosanit Pregl. 2020;77(3):335-9. https://doi.org/10.2298/NSP171128068Z.

51. Al-Turkait FA, Ohaeri JU. Prevalence and correlates of posttraumatic stress disorder among Kuwaiti military men according to level of involvement in the first gulf war. Depress Anxiety. 2008;25(11):932-41. https://doi.org/10.1 002/da.20373.

52. Kamm BA. Depressive, aggressive and paranoid reactions. Psychoanal Rev. 1951;38(2):127-38

53. Fisch M. The suicidal gesture: a study of 114 military patients hospitalized because of abortive suicide attempts. Am J Psychiatr. 1954;111(1):33-6. https://doi.org/10.1176/ajp.111.1.33.

54. OFFENKRANTZ W, CHURCH E, ELLIOTT R. Psychiatric management of suicide problems in military service. Am J Psychiatr. 1957;114(1):33-41. https://doi. org/10.1176/ajp.114.1.33.

55. Drehner D, Neuhauser KM, Neuhauser TS, Drehner D, Blackwood GV. Death among US air force basic trainees, 1956 to 1996. Mil Med. 1999;164(12):8417. https://doi.org/10.1093/milmed/164.12.841.
56. Slu P. Mental disorders in men enlisted into military service and in soldiers (clinico-epidemiological study). Zhurnal nevrologii i psikhiatrii imeni SS Korsakova. 1999;99(8):16-20.

57. Peltzer K. Trauma and mental health problems of Sudanese refugees in Uganda. Cent Afr J Med. 1999;45(5):110-4. https://doi.org/10.4314/cajm.v4 $5 i 5.8465$.

58. Rossow I, Romelsjo A, Leifman $\mathrm{H}$. Alcohol abuse and suicidal behaviour in young and middle aged men: differentiating between attempted and completed suicide. Addiction. 1999;94(8):1199-207. https://doi.org/10.1046/ j.1360-0443.1999.948119910.x.

59. Stahre MA, Brewer RD, Fonseca VP, Naimi TS. Binge drinking among US active-duty military personnel. Am J Prev Med. 2009;36(3):208-17. https:// doi.org/10.1016/j.amepre.2008.10.017.

60. Ames GM, Cunradi CB, Moore RS, Stern P. Military culture and drinking behavior among U.S. navy careerists. J Stud Alcohol Drugs. 2007:68(3):33644. https://doi.org/10.15288/jsad.2007.68.336.

61. Bray RM, Hourani LL. Substance use trends among active duty military personnel: findings from the United States Department of Defense Health Related Behavior Surveys, 1980-2005. Addiction. 2007;102(7):1092-101. https://doi.org/10.1111/j.1360-0443.2007.01841.x.

62. Grønkjaer $\mathrm{M}$, et al. Intelligence test scores before and after alcohol-related disorders-a longitudinal study of Danish male conscripts. Alcohol Clin Exp Res. 2019;43(10):2187-95. https://doi.org/10.1111/acer.14174.

63. Young $C M$, Pedersen ER, Pearson AD, Neighbors C. Drinking to cope moderates the efficacy of changing veteran drinking norms as a strategy for reducing drinking and alcohol-related problems among U.S. veterans. Psychol Addict Behav. 2018;32(2): 213-23. https://doi.org/10.1037/adb0000347.

64. Derefinko KJ, Klesges RC, Bursac Z, Little MA, Hryshko-Mullen A, Talcott GW. Alcohol issues prior to training in the United States air Force. Addict Behav. 2016;58:142-8. https://doi.org/10.1016/j.addbeh.2016.02.028.

65. Wang L, Seelig A, Wadsworth SMD, McMaster H, Alcaraz JE, Crum-Cianflone NF. Associations of military divorce with mental, behavioral, and physical health outcomes. BMC Psychiatry. 2015;15(1):128. https://doi.org/10.1186/s12 888-015-0517-7.

66. Williams EC, Frasco MA, Jacobson IG, Maynard C, Littman AJ, Seelig AD, et al. Risk factors for relapse to problem drinking among current and former US military personnel: a prospective study of the millennium cohort. Drug Alcohol Depend. 2015;148:93-101. https://doi.org/10.1016/j.drugalcdep.2 014.12.031.

67. Bray RM, Brown JM, Williams J. Trends in binge and heavy drinking, alcoholrelated problems, and combat exposure in the U.S. military. Subst Use Misuse. 2013;48(10):799-810. https://doi.org/10.3109/10826084.2013.796990.

68. Farsi Z, JABARI MM, Saghiri Z. The relationship between depression with self injury in Army soldiers seen in a military medical outpatient Clinic in Tehran; 2010.

69. Rohani, S., et al., The frequency of suicidal-thinking in NEZAJA personnel in Tehran at 2004-5 year. 2006

\section{Publisher's Note}

Springer Nature remains neutral with regard to jurisdictional claims in published maps and institutional affiliations.

Ready to submit your research? Choose BMC and benefit from:

- fast, convenient online submission

- thorough peer review by experienced researchers in your field

- rapid publication on acceptance

- support for research data, including large and complex data types

- gold Open Access which fosters wider collaboration and increased citations

- maximum visibility for your research: over $100 \mathrm{M}$ website views per year

At BMC, research is always in progress.

Learn more biomedcentral.com/submission 